ESAIM: COCV 27 (2021) 81

https://doi.org/10.1051/cocv/2021078
ESAIM: Control, Optimisation and Calculus of Variations

www.esaim-cocv.org

\title{
MEAN-FIELD LINEAR-QUADRATIC STOCHASTIC DIFFERENTIAL GAMES IN AN INFINITE HORIZON*
}

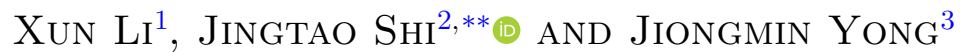

\begin{abstract}
This paper is concerned with two-person mean-field linear-quadratic non-zero sum stochastic differential games in an infinite horizon. Both open-loop and closed-loop Nash equilibria are introduced. The existence of an open-loop Nash equilibrium is characterized by the solvability of a system of mean-field forward-backward stochastic differential equations in an infinite horizon and the convexity of the cost functionals, and the closed-loop representation of an open-loop Nash equilibrium is given through the solution to a system of two coupled non-symmetric algebraic Riccati equations. The existence of a closed-loop Nash equilibrium is characterized by the solvability of a system of two coupled symmetric algebraic Riccati equations. Two-person mean-field linear-quadratic zero-sum stochastic differential games in an infinite horizon are also considered. Both the existence of open-loop and closed-loop saddle points are characterized by the solvability of a system of two coupled generalized algebraic Riccati equations with static stabilizing solutions. Mean-field linear-quadratic stochastic optimal control problems in an infinite horizon are discussed as well, for which it is proved that the open-loop solvability and closed-loop solvability are equivalent.
\end{abstract}

Mathematics Subject Classification. 91A15, 91A16, 91A23, 93C05, 93E20, 49N10.

Received July 13, 2020. Accepted July 3, 2021.

\section{INTRODUCTION}

Let $(\Omega, \mathcal{F}, \mathbb{P}, \mathbb{F})$ be a complete filtered probability space, on which a one-dimensional standard Brownian motion $W(\cdot)$ is defined with $\mathbb{F} \equiv\left\{\mathcal{F}_{t}\right\}_{t \geqslant 0}$ being its natural filtration augmented by all the $\mathbb{P}$-null sets in $\mathcal{F}$, and $\mathbb{E}[\cdot]$ denotes the expectation with respect to $\mathbb{P}$. Throughout this paper, we let $\mathbb{R}^{n \times m}$ and $\mathbb{S}^{n}$ be the set of all $(n \times m)$ (real) matrices and $(n \times n)$ symmetric (real) matrices. We denote $\mathbb{R}^{n}=\mathbb{R}^{n \times 1}$. For a Euclidean space $\mathbb{H}$, say, $\mathbb{H}=\mathbb{R}^{n}, \mathbb{R}^{n \times m}$, let $C([0, \infty) ; \mathbb{H})$ denote the space of $\mathbb{H}$-valued continuous functions $\varphi:[0, \infty) \rightarrow \mathbb{H}, L^{2}(\mathbb{H})$ denote the space of $\mathbb{H}$-valued functions $\varphi:[0, \infty) \rightarrow \mathbb{H}$ with $\int_{0}^{\infty}|\varphi(t)|^{2} \mathrm{~d} t<\infty$, and $L_{\mathbb{F}}^{2}(\mathbb{H})$ denote the space of $\mathbb{F}$-progressively measurable processes $\varphi:[0, \infty) \times \Omega \rightarrow \mathbb{H}$ with $\mathbb{E} \int_{0}^{\infty}|\varphi(t)|^{2} \mathrm{~d} t<\infty$.

* This work was financially supported by Research Grants Council of Hong Kong under Grant 15213218 and 15215319 , National Key R\&D Program of China under Grant 2018YFB1305400, National Natural Science Funds of China under Grant 11971266, 11831010 and 11571205, China Scholarship Council, Shandong Provincial Natural Science Foundations under Grant ZR2020ZD24 and ZR2019ZD42, and NSF Grant DMS-1812921.

Keywords and phrases: Two-person mean-field linear-quadratic stochastic differential game, infinite horizon, open-loop and closed-loop Nash equilibria, algebraic Riccati equations, MF- $L^{2}$-stabilizability, static stabilizing solution.

1 Department of Applied Mathematics, Hong Kong Polytechnic University, Hong Kong, China.

2 School of Mathematics, Shandong University, Jinan 250100, China.

3 Department of Mathematics, University of Central Florida, Orlando, FL 32816, USA.

** Corresponding author: shijingtao@sdu.edu.cn 
Consider the following controlled linear mean-field stochastic differential equation (MF-SDE, for short) on an infinite horizon $[0, \infty)$ :

$$
\left\{\begin{aligned}
\mathrm{d} X(t)= & \left\{A X(t)+\bar{A} \mathbb{E}[X(t)]+B_{1} u_{1}(t)+\bar{B}_{1} \mathbb{E}\left[u_{1}(t)\right]+B_{2} u_{2}(t)+\bar{B}_{2} \mathbb{E}\left[u_{2}(t)\right]+b(t)\right\} \mathrm{d} t \\
& +\left\{C X(t)+\bar{C} \mathbb{E}[X(t)]+D_{1} u_{1}(t)+\bar{D}_{1} \mathbb{E}\left[u_{1}(t)\right]+D_{2} u_{2}(t)+\bar{D}_{2} \mathbb{E}\left[u_{2}(t)\right]+\sigma(t)\right\} \mathrm{d} W(t), t \geqslant 0, \\
X(0)= & x
\end{aligned}\right.
$$

In the above, $X(\cdot)$ is the state process taking values in $\mathbb{R}^{n}$ with $x$ being the initial state. For $i=1,2, u_{i}(\cdot)$ is the control process of Player $i$, taking values in $\mathbb{R}^{m_{i}}$, respectively. The coefficients $A, \bar{A}, C, \bar{C} \in \mathbb{R}^{n \times n}$, $B_{1}, \bar{B}_{1}, D_{1}, \bar{D}_{1} \in \mathbb{R}^{n \times m_{1}}, B_{2}, \bar{B}_{2}, D_{2}, \bar{D}_{2} \in \mathbb{R}^{n \times m_{2}}$ are given constant matrices, and the non-homogenous terms $b(\cdot), \sigma(\cdot) \in L_{\mathbb{F}}^{2}\left(\mathbb{R}^{n}\right)$. We introduce the following spaces:

$$
\left\{\begin{aligned}
& \mathscr{X}[0, T]=\left\{X:[0, \infty) \times \Omega \rightarrow \mathbb{R}^{n} \mid X(\cdot) \text { is } \mathbb{F} \text {-adapted, } t \mapsto X(t, \omega)\right. \text { is continuous, and } \\
&\left.\mathbb{E}\left[\sup _{t \in[0, T]}|X(t)|^{2}\right]<\infty\right\}, \text { for } T>0, \\
& \mathscr{X}_{l o c}[0, \infty)=\bigcap_{T>0} \mathscr{X}[0, T], \quad \mathscr{X}[0, \infty)=\left\{\left.X(\cdot) \in \mathscr{X}_{l o c}[0, \infty)\left|\mathbb{E} \int_{0}^{\infty}\right| X(t)\right|^{2} \mathrm{~d} t<\infty\right\} .
\end{aligned}\right.
$$

By a standard argument using contraction mapping theorem, one can show that for any initial state $x \in \mathbb{R}^{n}$ and control pair $\left(u_{1}(\cdot), u_{2}(\cdot)\right) \in L_{\mathbb{F}}^{2}\left(\mathbb{R}^{m_{1}}\right) \times L_{\mathbb{F}}^{2}\left(\mathbb{R}^{m_{2}}\right)$, state equation (1.1) admits a unique strong solution $X(\cdot) \equiv X\left(\cdot ; x, u_{1}(\cdot), u_{2}(\cdot)\right) \in \mathscr{X}_{l o c}[0, \infty)$. Next, for $i=1,2$, we introduce the following cost functionals:

$$
J_{i}\left(x ; u_{1}(\cdot), u_{2}(\cdot)\right)=\mathbb{E} \int_{0}^{\infty} g_{i}\left(t, X(t), u_{1}(t), u_{2}(t), \mathbb{E}[X(t)], \mathbb{E}\left[u_{1}(t)\right], \mathbb{E}\left[u_{2}(t)\right]\right) \mathrm{d} t,
$$

with

$$
\begin{aligned}
g_{i}\left(t, x, u_{1}, u_{2}, \bar{x}, \bar{u}_{1}, \bar{u}_{2}\right)= & \left\langle\left(\begin{array}{ccc}
Q_{i} & S_{i 1}^{\top} & S_{i 2}^{\top} \\
S_{i 1} & R_{i 11} & R_{i 12} \\
S_{i 2} & R_{i 21} & R_{i 22}
\end{array}\right)\left(\begin{array}{c}
x \\
u_{1} \\
u_{2}
\end{array}\right),\left(\begin{array}{c}
x \\
u_{1} \\
u_{2}
\end{array}\right)\right\rangle+2\left\langle\left(\begin{array}{c}
q_{i}(t) \\
\rho_{i 1}(t) \\
\rho_{i 2}(t)
\end{array}\right),\left(\begin{array}{c}
x \\
u_{1} \\
u_{2}
\end{array}\right)\right\rangle \\
& +\left\langle\left(\begin{array}{ccc}
\bar{Q}_{i} & \bar{S}_{i 1}^{\top} & \bar{S}_{i 2}^{\top} \\
\bar{S}_{i 1} & \bar{R}_{i 11} & \bar{R}_{i 12} \\
\bar{S}_{i 2} & \bar{R}_{i 21} & \bar{R}_{i 22}
\end{array}\right)\left(\begin{array}{c}
\bar{x} \\
\bar{u}_{1} \\
\bar{u}_{2}
\end{array}\right),\left(\begin{array}{c}
\bar{x} \\
\bar{u}_{1} \\
\bar{u}_{2}
\end{array}\right)\right\rangle,
\end{aligned}
$$

where

$$
\left\{\begin{array}{lll}
Q_{i}, \bar{Q}_{i} \in \mathbb{S}^{n}, & S_{i 1}, \bar{S}_{i 1} \in \mathbb{R}^{m_{1} \times n}, & S_{i 2}, \bar{S}_{i 2} \in \mathbb{R}^{m_{2} \times n}, \\
R_{i 11}, \bar{R}_{i 11} \in \mathbb{S}^{m_{1}}, & R_{i 22}, \bar{R}_{i 22} \in \mathbb{S}^{m_{2}}, & R_{i 12}=R_{i 21}^{\top}, \bar{R}_{i 12}=\bar{R}_{i 21}^{\top} \in \mathbb{R}^{m_{1} \times m_{2}}, \\
q_{i}(\cdot) \in L_{\mathbb{F}}^{2}\left(\mathbb{R}^{n}\right), & \rho_{i 1}(\cdot) \in L_{\mathbb{F}}^{2}\left(\mathbb{R}^{m_{1}}\right), & \rho_{i 2}(\cdot) \in L_{\mathbb{F}}^{2}\left(\mathbb{R}^{m_{2}}\right) .
\end{array}\right.
$$

Note that for $\left(x, u_{1}(\cdot), u_{2}(\cdot)\right) \in \mathbb{R}^{n} \times L_{\mathbb{F}}^{2}\left(\mathbb{R}^{m_{1}}\right) \times L_{\mathbb{F}}^{2}\left(\mathbb{R}^{m_{2}}\right)$, the solution $X(\cdot) \equiv X\left(\cdot ; x, u_{1}(\cdot), u_{2}(\cdot)\right)$ to $(1.1)$ might just be in $\mathscr{X}_{l o c}[0, \infty)$ in general. Therefore, in order the cost functionals $J_{i}\left(x ; u_{1}(\cdot), u_{2}(\cdot)\right), i=1,2$ to be defined, the control pair $\left(u_{1}(\cdot), u_{2}(\cdot)\right)$ has to be restricted in the following set of admissible control pairs:

$$
\mathscr{U}_{a d}(x)=\left\{\left(u_{1}(\cdot), u_{2}(\cdot)\right) \in L_{\mathbb{F}}^{2}\left(\mathbb{R}^{m_{1}}\right) \times L_{\mathbb{F}}^{2}\left(\mathbb{R}^{m_{2}}\right) \mid X\left(\cdot ; x, u_{1}(\cdot), u_{2}(\cdot)\right) \in \mathscr{X}[0, \infty)\right\}, \quad x \in \mathbb{R}^{n} .
$$


Note that $\mathscr{U}_{a d}(x)$ depends on the initial state $x$. For any $\left(u_{1}(\cdot), u_{2}(\cdot)\right) \in \mathscr{U}_{a d}(x)$, the corresponding $X(\cdot) \equiv$ $X\left(\cdot ; x, u_{1}(\cdot), u_{2}(\cdot)\right)$ is called an admissible state process for the initial state $x$. Then we can loosely formulate the following problem.

Problem (MF-SDG). For any initial state $x \in \mathbb{R}^{n}$, Player $i(i=1,2)$ wants to find a control $u_{i}^{*}(\cdot)$ so that $\left(u_{1}^{*}(\cdot), u_{2}^{*}(\cdot)\right) \in \mathscr{U}_{a d}(x)$ such that the cost functionals $u_{1}(\cdot) \mapsto J_{1}\left(x ; u_{1}(\cdot), u_{2}^{*}(\cdot)\right)$ and $u_{2}(\cdot) \mapsto J_{2}\left(x ; u_{1}^{*}(\cdot), u_{2}(\cdot)\right)$ are minimized, for all $\left(u_{1}(\cdot), u_{2}^{*}(\cdot)\right),\left(u_{1}^{*}(\cdot), u_{2}(\cdot)\right) \in \mathscr{U}_{a d}(x)$, subject to (1.1).

We refer to the above problem as a mean-field linear-quadratic (LQ, for short) two-person (non-zero sum) stochastic differential game in an infinite horizon. In the special case where $b(\cdot), \sigma(\cdot), q_{i}(\cdot), \rho_{i j}(\cdot)$ are all zero, we denote the corresponding problem and cost functionals by Problem (MF-SDG) ${ }^{0}$ and $J_{i}^{0}\left(x ; u_{1}(\cdot), u_{2}(\cdot)\right)$, respectively. On the other hand, when

$$
J_{1}\left(x ; u_{1}(\cdot), u_{2}(\cdot)\right)+J_{2}\left(x ; u_{1}(\cdot), u_{2}(\cdot)\right)=0, \quad \forall x \in \mathbb{R}^{n}, \forall\left(u_{1}(\cdot), u_{2}(\cdot)\right) \in \mathscr{U}_{a d}(x),
$$

the corresponding Problem (MF-SDG) is called a mean-field LQ two-person zero-sum stochastic differential game in an infinite horizon and is denoted by Problem (MF-SDG) $)_{0}$. To guarantee (1.5), one usually lets

$$
\left\{\begin{array}{lll}
Q_{1}+Q_{2}=0, & \bar{Q}_{1}+\bar{Q}_{2}=0, & q_{1}(\cdot)+q_{2}(\cdot)=0, \\
S_{1 j}+S_{2 j}=0, & \bar{S}_{1 j}+\bar{S}_{2 j}=0, & \rho_{1 j}(\cdot)+\rho_{2 j}(\cdot)=0, \\
R_{1 j k}+R_{2 j k}=0, & \bar{R}_{1 j k}+\bar{R}_{2 j k}=0, & j, k=1,2 .
\end{array}\right.
$$

One may feel that cost functional (1.2) could be a little more general by including terms like $\left\langle\bar{q}_{i}(s), \mathbb{E}[X(s)]\right\rangle$. However, it is not hard to see that (as long as the integrals exist)

$$
\mathbb{E} \int_{0}^{\infty}\left\langle\bar{q}_{i}(s), \mathbb{E}[X(s)]\right\rangle \mathrm{d} s=\mathbb{E} \int_{0}^{\infty}\left\langle\mathbb{E}\left[\bar{q}_{i}(s)\right], X(s)\right\rangle \mathrm{d} s,
$$

which can be absorbed by replacing $q_{i}(\cdot)$ by $q_{i}(\cdot)+\mathbb{E}\left[\bar{q}_{i}(\cdot)\right]$ in the cost functionals. Likewise, terms like $\left\langle\bar{\rho}_{i j}(s), \mathbb{E}\left[u_{j}(s)\right]\right\rangle$ are not necessarily included.

We will introduce proper stabilizability conditions for the system so that $\mathscr{U}_{a d}(x) \neq \varnothing$ for all $x \in \mathbb{R}^{n}$. It is not hard to see that without stabilizabity conditions, one might only has $X\left(\cdot ; x, u_{1}(\cdot), u_{2}(\cdot)\right) \in \mathscr{X}_{\text {loc }}[0, \infty)$ so that $J_{i}\left(x ; u_{1}(\cdot), u_{2}(\cdot)\right)$ might not be well-defined. Then, instead of considering the cost functionals of form (1.2), one might naturally consider the following ergodic type cost functionals:

$$
\widetilde{J}_{i}\left(x ; u_{1}(\cdot), u_{2}(\cdot)\right)=\varlimsup_{T \rightarrow \infty} \frac{1}{T} \int_{0}^{T} g_{i}\left(t, X(t), u_{1}(t), u_{2}(t), \mathbb{E}[X(t)], \mathbb{E}\left[u_{1}(t)\right], \mathbb{E}\left[u_{2}(t)\right]\right) \mathrm{d} t .
$$

The above type cost functionals are normally used for the case that the running cost rate is bounded so that the right-hand side of the above is always finite (see, for example, Cohen-Fedyashov [18]). However, if no stabilizability conditions are assumed, the state process $X(\cdot)$ could be of exponential growth. In that case, the above type cost functionals are still not useful. Therefore, instead of (1.7), we prefer to study our problems under certain stabilizability condition with cost functionals of for (1.2) restricted on $\mathscr{U}_{a d}(x)$.

The theory of MF-SDEs can be traced back to the work of Kac [25] in the middle of 1950s, where a stochastic toy model for the Vlasov type kinetic equation of plasma was presented. Its rigorous study was initiated by McKean [32] in 1966, which is now known as McKean-Vlasov stochastic differential equations. Since then, many researchers have made contributions to the related topics and their applications; see, for example, Scheutzow [40], Chan [17], Huang-Malhame-Caines [24], Buckdahn-Li-Peng [13], Carmona-Delarue [15], BensoussanYam-Zhang [9], Buckdahn-Li-Peng-Rainer [14], etc.

Optimal control and differential game problems of MF-SDEs have drawn enormous researchers' attention recently. See Ahmed-Ding [3], Lasry-Lions [26], Andersson-Djehiche [4], Buckdahn-Djehiche-Li [10], Li [27], 
Meyer-Brandis-Oksendal-Zhou [33], Hosking [21], Bensoussan-Sung-Yam [7], Djehiche-Tembine-Tempone [20], Bensoussan-Sung-Yam-Yung [8], Djehiche-Tembine [19], Huang-Li-Wang [22], Yong [54], Buckdahn-Li-Ma [11, 12], Pham-Wei [37, 38], Li-Sun-Xiong [30], Miller-Pham [34], Moon [35], and the references therein. Next, let us mention a few recent pieces of literature related to our present paper. In Yong [53], an LQ optimal control problem for MF-SDEs in a finite horizon was introduced and investigated. The optimality system of a linear mean-field forward-backward stochastic differential equation (MF-FBSDE, for short) is derived, and two Riccati differential equations are obtained to present the feedback representation of the optimal control. Huang-LiYong [23] generalized the results in [53] to the infinite horizon case, and the feedback representation of the optimal control is derived via two algebraic Riccati equations (AREs, for short). Note that in [23], some notions of stabilizability for controlled MF-SDEs are introduced, which are interestingly different from the classical ones, due to the presence of the terms $\mathbb{E}[X(\cdot)]$ and $\mathbb{E}[u(\cdot)]$. Sun [41] continued to investigate the LQ optimal control problem for MF-SDEs in the finite horizon with additional nonhomogeneous terms and concluded that the uniform convexity of the cost functional is sufficient for the open-loop solvability of the LQ optimal control problems for MF-SDEs. Moreover, the uniform convexity of the cost functional is equivalent to the solvability of two coupled differential Riccati equations, and the unique open-loop optimal control admits a state feedback representation in the case that the cost functional is uniformly convex. Li-Sun-Yong [31] studied the closedloop solvability of the corresponding problem, which is characterized by the existence of a regular solution to the coupled two generalized Riccati equations, together with some constraints on the adapted solution to a linear backward stochastic differential equation (BSDE, for short) and a linear terminal value problem of an ordinary differential equation (ODE, for short). Li-Li-Yu [28] analyzed the indefinite mean-field type LQ stochastic optimal control problems, where they introduced a relaxed compensator to characterize the openloop solvability of the problem. Tian-Yu-Zhang [51] considered an LQ zero-sum stochastic differential game with mean-field type, proposed the notions of explicit and implicit strategy laws, and established the closed-loop formulation for saddle points in the mixed-strategy-law. Very recently, Sun-Wang-Wu [44] studied a two-person zero-sum mean-field LQ stochastic differential game over the finite horizon by a Hilbert space method introduced by Mou-Yong [36]. It is shown that the associated two Riccati equations admit unique and strongly regular solutions under the sufficient condition for the existence of an open-loop saddle point when the open-loop saddle point can be represented as linear feedback of the current state. When only the necessary condition for the existence of an open-loop saddle point is satisfied, we can construct an approximate sequence by solving a family of Riccati equations and closed-loop systems. The approximate sequence's convergence turns out to be equivalent to the open-loop solvability of the game, and its limit exactly equals an open-loop saddle point, provided that the game is open-loop solvable.

Ait Rami-Zhou [1] and Ait Rami-Zhou-Moore [2] considered stochastic LQ problems in an infinite horizon, with indefinite control weighting matrices. They introduced a generalized ARE, involving a matrix pseudoinverse and two additional algebraic equality/inequality constraints, and proved that the problem's attainability is equivalent to the existence of a static stabilizing solution to the generalized ARE. In particular, the associated AREs can be solved by linear matrix inequality and semidefinite programming techniques. In addition to the statements in the previous paragraph about [23], the authors discussed the solvabilities of AREs, by linear matrix inequalities. Sun-Yong [46] first found that both the open-loop and closed-loop solvabilities of the stochastic LQ problems in the infinite horizon are equivalent to the existence of a static stabilizing solution to the associated generalized ARE. We refer the readers to the recent monographs by Bensoussan-Frehse-Yam [6] and by Sun-Yong $[48,49]$ for more details and references cited therein.

In this paper, we consider two-person mean-field LQ non-zero sum stochastic differential games in an infinite horizon. Both open-loop and closed-loop Nash equilibria are introduced. The existence of an open-loop Nash equilibrium is characterized by the solvability of a system of MF-FBSDEs in an infinite horizon and the convexity of the cost functionals. The closed-loop representation of an open-loop Nash equilibrium is given through the solution to a system of two coupled non-symmetric AREs. The existence of a closed-loop Nash equilibrium is characterized by the solvability of a system of two coupled symmetric AREs. Two-person mean-field LQ zero-sum stochastic differential games in an infinite horizon are also considered. The existence of open-loop and closed-loop saddle points is characterized by the solvability of a system of two coupled generalized AREs with 
static stabilizing solutions. As special cases, mean-field LQ stochastic optimal control problems in an infinite horizon are discussed as well, for which it is proved that the open-loop solvability and closed-loop solvability are equivalent. The results obtained in this paper enrich the theory of optimal control and differential games of mean-field type.

Let us briefly highlight the major novelty of this paper:

(i) For MF-SDEs with quadratic performance indexes in an infinite-horizon, problems of two-person non-zero sum differential games, two-person zero-sum differential games and optimal control (which is a single player differential game) are treated in a unified framework. Among other results, the most significant one is the discovery of the system of coupled algebraic Riccati equations (3.35) which is used to characterize the closedloop Nash equilibrium. From this point of view, the current paper can be regarded as a complementary or a continuation of Sun-Yong [47].

(ii) For MF-SDE LQ optimal control problems in an infinite horizon, we have established the equivalence among the solvability of a system of coupled algebraic Riccati equations, open-loop solvability, and closed-loop solvability. This covers the relevant results found in Sun-Yong [46] where mean-field terms were absent.

(iii) For two-person zero-sum differential games of MF-SDEs with quadratic performance index in $[0, \infty)$, we have proved that if an open-loop saddle point admits a closed-loop representation, and the closed-loop saddle point exists, then the open-loop saddle point must be the outcome of the closed-loop saddle point. It is also shown that such a property fails for non-zero sum differential games. From this angle, the current paper is an extension of Sun-Yong-Zhang [50] where the mean-field terms did not appear.

The rest of the paper is organized as follows. In Section 2, we present some preliminary results about mean-field LQ stochastic optimal control problems in an infinite horizon. Section 3 aims to give results on mean-field LQ non-zero sum stochastic differential games, including open-loop Nash equilibria and their closedloop representation, and closed-loop Nash equilibria with algebraic Riccati equations. In Section 4, the open-loop and closed-loop saddle points for mean-field LQ zero-sum stochastic differential games are investigated. Some examples are presented in Section 5 illustrating the results developed in the earlier sections. Finally, some concluding remarks are collected in Section 6.

\section{Preliminaries}

Throughout this paper, besides the notation introduced in the previous section, we let $I$ be the identity matrix or operator with a suitable size. We will use $\langle\cdot, \cdot\rangle$ for inner products in possibly different Hilbert spaces, and denote by $|\cdot|$ the norm induced by $\langle\cdot, \cdot\rangle$. Let $M^{\top}$ and $\mathscr{R}(M)$ be the transpose and range of a matrix $M$, respectively. For $M, N \in \mathbb{S}^{n}$, we write $M \geqslant N$ (respectively, $M>N$ ) for $M-N$ being positive semi-definite (respectively, positive definite). Let $M^{\dagger}$ denote the pseudo-inverse of a matrix $M \in \mathbb{R}^{m \times n}$, which is equal to the inverse $M^{-1}$ of $M \in \mathbb{R}^{n \times n}$ if it exists. See Penrose [39] or Anderson-Moore [5] for some basic properties of the pseudo-inverse. We define the inner product in $L_{\mathbb{F}}^{2}(\mathbb{H})$ by $\langle\varphi, \phi\rangle=\mathbb{E} \int_{0}^{\infty}\langle\varphi(t), \phi(t)\rangle \mathrm{d} t$ so that $L_{\mathbb{F}}^{2}(\mathbb{H})$ is a Hilbert space.

We now consider the following controlled linear MF-SDE over $[0, \infty)$ :

$$
\left\{\begin{array}{l}
\begin{array}{rl}
\mathrm{d} X(t)=\{A X(t)+\bar{A} \mathbb{E}[X(t)]+B u(t)+\bar{B} \mathbb{E}[u(t)]+b(t)\} \mathrm{d} t \\
\quad+\{C X(t)+\bar{C} \mathbb{E}[X(t)]+D u(t)+\bar{D} \mathbb{E}[u(t)]+\sigma(t)\} \mathrm{d} W(t), \quad t \geqslant 0,
\end{array} \\
X(0)=x,
\end{array}\right.
$$

with quadratic cost functional

$$
\begin{aligned}
J(x ; u(\cdot))=\mathbb{E} \int_{0}^{\infty} & {\left[\left\langle\left(\begin{array}{cc}
Q & S^{\top} \\
S & R
\end{array}\right)\left(\begin{array}{c}
X(t) \\
u(t)
\end{array}\right),\left(\begin{array}{c}
X(t) \\
u(t)
\end{array}\right)\right\rangle+2\left\langle\left(\begin{array}{c}
q(t) \\
\rho(t)
\end{array}\right),\left(\begin{array}{c}
X(t) \\
u(t)
\end{array}\right)\right\rangle\right.} \\
+ & \left.\left\langle\left(\begin{array}{cc}
\bar{Q} & \bar{S}^{\top} \\
\bar{S} & \bar{R}
\end{array}\right)\left(\begin{array}{c}
\mathbb{E}[X(t)] \\
\mathbb{E}[u(t)]
\end{array}\right),\left(\begin{array}{c}
\mathbb{E}[X(t)] \\
\mathbb{E}[u(t)]
\end{array}\right)\right\rangle\right] \mathrm{d} t
\end{aligned}
$$


where $A, \bar{A}, C, \bar{C} \in \mathbb{R}^{n \times n}, B, \bar{B}, D, \bar{D} \in \mathbb{R}^{n \times m}, Q, \bar{Q} \in \mathbb{S}^{n}, S, \bar{S} \in \mathbb{R}^{m \times n}, R, \bar{R} \in \mathbb{S}^{m}$ are given constant matrices, and $b(\cdot), \sigma(\cdot), q(\cdot) \in L_{\mathbb{F}}^{2}\left(\mathbb{R}^{n}\right), \rho(\cdot) \in L_{\mathbb{F}}^{2}\left(\mathbb{R}^{m}\right)$ are stochastic processes. For any initial state $x \in \mathbb{R}^{n}$ and control $u(\cdot) \in L_{\mathbb{F}}^{2}\left(\mathbb{R}^{m}\right)$, equation $(2.1)$ admits a unique strong solution $X(\cdot) \equiv X(\cdot ; x, u(\cdot)) \in \mathscr{X}_{\text {loc }}[0, \infty)$. We define the admissible control set as

$$
\mathscr{U}_{a d}(x)=\left\{u(\cdot) \in L_{\mathbb{F}}^{2}\left(\mathbb{R}^{m}\right) \mid X(\cdot) \equiv X(\cdot ; x, u(\cdot)) \in \mathscr{X}[0, \infty)\right\}
$$

In general, $\mathscr{U}_{a d}(x)$ depends on $x \in \mathbb{R}^{n}$. Let us pose the following optimal control problem.

Problem (MF-SLQ). For any initial state $x \in \mathbb{R}^{n}$, find a control $u^{*}(\cdot) \in \mathscr{U}_{a d}(x)$ such that the cost functional $J(x ; u(\cdot))$ of $(2.2)$ is minimized, subject to $(2.1)$. That is to say,

$$
J\left(x ; u^{*}(\cdot)\right)=\inf _{u(\cdot) \in \mathscr{U}_{a d}(x)} J(x ; u(\cdot)) \equiv V(x) .
$$

Any $u^{*}(\cdot) \in \mathscr{U}_{a d}(x)$ satisfying (2.4) is called an open-loop optimal control of Problem (MF-SLQ), and the corresponding $X^{*}(\cdot) \equiv X\left(\cdot ; x, u^{*}(\cdot)\right)$ is called an open-loop optimal state process. The function $V(\cdot)$ is called the value function of Problem (MF-SLQ). In the special case where $b(\cdot), \sigma(\cdot), q(\cdot), \rho(\cdot)$ are all zero, we denote the corresponding problem by Problem (MF-SLQ) $)^{0}$, the cost functional by $J^{0}(x ; u(\cdot))$ and the value function by $V^{0}(x)$, respectively.

In order Problem (MF-SLQ) to be meaningful, we need to find conditions under which $\mathscr{U}_{a d}(x)$ is nonempty and admits an accessible characterization. For this target, let us first look at the following uncontrolled non-homogeneous linear system on $[0, \infty)$ :

$$
\left\{\begin{array}{l}
\mathrm{d} X(t)=\{A X(t)+\bar{A} \mathbb{E}[X(t)]+b(t)\} \mathrm{d} t+\{C X(t)+\bar{C} \mathbb{E}[X(t)]+\sigma(t)\} \mathrm{d} W(t), \quad t \geqslant 0, \\
X(0)=x
\end{array}\right.
$$

When $b(\cdot)=\sigma(\cdot)=0$, the system is said to be homogeneous and denoted by $[A, \bar{A}, C, \bar{C}]$. For simplicity, we also denote $[A, C]=[A, 0, C, 0]$ (the linear SDE without mean-fields), and $A=[A, 0]=[A, 0,0,0]$ (the linear ordinary differential equation, ODE, for short). The following notions can be found in [23].

Definition 2.1. (i) System $[A, \bar{A}, C, \bar{C}]$ is said to be $L^{2}$-globally integrable, if for any $x \in \mathbb{R}^{n}$, the solution $X(\cdot) \equiv X(\cdot ; x)$ of $(2.5)$ with $b(\cdot)=\sigma(\cdot)=0$ is in $\mathscr{X}[0, \infty)$.

(ii) System $[A, \bar{A}, C, \bar{C}]$ is said to be $L^{2}$-asymptotically stable, if for any $x \in \mathbb{R}^{n}$, the solution $X(\cdot) \equiv X(\cdot ; x) \in$ $\mathscr{X}_{\text {loc }}[0, \infty)$ of $(2.5)$ with $b(\cdot)=\sigma(\cdot)=0$ satisfies $\lim _{t \rightarrow \infty} \mathbb{E}|X(t)|^{2}=0$.

According to [23], and via a similar argument proving Theorem 3.3 of [50], we have the following result.

Proposition 2.2. For any $x \in \mathbb{R}^{n}$ and $b(\cdot), \sigma(\cdot) \in L_{\mathbb{F}}^{2}\left(\mathbb{R}^{n}\right)$, linear MF-SDE (2.5) admits a unique solution $X(\cdot) \in \mathscr{X}_{\text {loc }}[0, \infty)$. Further, if $[A, \bar{A}, C, \bar{C}]$ is $L^{2}$-asymptotically stable and system $[A, C]$ is $L^{2}$-globally integrable, then $X(\cdot) \in \mathscr{X}[0, \infty)$, with

$$
\mathbb{E} \int_{0}^{\infty}|X(t)|^{2} \mathrm{~d} t \leqslant K\left[|x|^{2}+\mathbb{E} \int_{0}^{\infty}\left(|b(t)|^{2}+|\sigma(t)|^{2}\right) \mathrm{d} t\right]
$$

for some constant $K>0$. On the other hand, for any $\varphi(\cdot) \in L_{\mathbb{F}}^{2}\left(\mathbb{R}^{n}\right)$, the following linear MF-BSDE:

$$
-\mathrm{d} Y(t)=\left\{A^{\top} Y(t)+\bar{A}^{\top} \mathbb{E}[Y(t)]+C^{\top} Z(t)+\bar{C}^{\top} \mathbb{E}[Z(t)]+\varphi(t)\right\} \mathrm{d} t-Z(t) \mathrm{d} W(t), \quad t \geqslant 0,
$$

admits a unique adapted solution $(Y(\cdot), Z(\cdot)) \in \mathscr{X}[0, \infty) \times L_{\mathbb{F}}^{2}\left(\mathbb{R}^{n}\right)$.

For general theory of MF-BSDEs and MF-FBSDEs in a finite horizon, see [13, 15, 16]. 
Now we return to (2.1). Similar to the above, when $b(\cdot)=\sigma(\cdot)=0$, the system is said to be homogeneous and denote it by $[A, \bar{A}, C, \bar{C} ; B, \bar{B}, D, \bar{D}]$.

Definition 2.3. (i) For any $\Theta \equiv(\Theta, \bar{\Theta}) \in \mathbb{R}^{m \times 2 n}$ and $v(\cdot) \in L_{\mathbb{F}}^{2}\left(\mathbb{R}^{m}\right)$,

$$
u(\cdot)=u^{\Theta, v}(\cdot) \equiv \Theta\{X(\cdot)-\mathbb{E}[X(\cdot)]\}+\bar{\Theta} \mathbb{E}[X(\cdot)]+v(\cdot) \equiv \Theta\left(\begin{array}{c}
X(\cdot)-\mathbb{E}[X(\cdot)] \\
\mathbb{E}[X(\cdot)]
\end{array}\right)+v(\cdot)
$$

is called a feedback control. Under such a control, the state equation (2.1) becomes

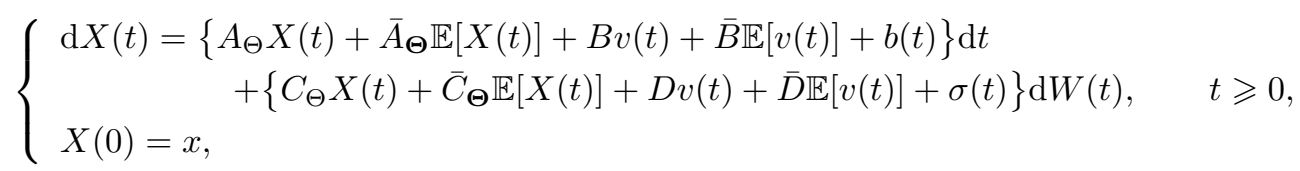

where

$$
A_{\Theta}=A+B \Theta, \quad \bar{A}_{\Theta}=\bar{A}+\bar{B} \bar{\Theta}+B(\bar{\Theta}-\Theta), \quad C_{\Theta}=C+D \Theta, \quad \bar{C}_{\Theta}=\bar{C}+\bar{D} \bar{\Theta}+D(\bar{\Theta}-\Theta) .
$$

(ii) System $[A, \bar{A}, C, \bar{C} ; B, \bar{B}, D, \bar{D}]$ is said to be MF- $L^{2}$-stabilizable, if there exists a $\Theta \equiv(\Theta, \bar{\Theta}) \in \mathbb{R}^{m \times 2 n}$ such that system $\left[A_{\Theta}, \bar{A}_{\Theta}, C_{\Theta}, \bar{C}_{\Theta}\right]$ is $L^{2}$-asymptotically stable and system $\left[A_{\Theta}, C_{\Theta}\right]$ is $L^{2}$-globally integrable. In this case, $\Theta \equiv(\Theta, \bar{\Theta})$ is called an MF- $L^{2}$-stabilizer of $[A, \bar{A}, C, \bar{C} ; B, \bar{B}, D, \bar{D}]$. The set of all MF- $L^{2}$-stabilizers of $[A, \bar{A}, C, \bar{C} ; B, \bar{B}, D, \bar{D}]$ is denoted by $\mathscr{S}[A, \bar{A}, C, \bar{C} ; B, \bar{B}, D, \bar{D}]$.

(iii) Any pair $(\Theta, v(\cdot)) \in \mathscr{S}[A, \bar{A}, C, \bar{C} ; B, \bar{B}, D, \bar{D}] \times L_{\mathbb{F}}^{2}\left(\mathbb{R}^{m}\right)$ is called a closed-loop strategy of Problem (MFSLQ). The solution $X(\cdot) \equiv X^{\boldsymbol{\Theta}, v}(\cdot)$ of $(2.8)$ is called the closed-loop state process corresponding to $(\boldsymbol{\Theta}, v(\cdot))$. The control $u(\cdot)$ defined by $(2.7)$ is called the outcome of $(\boldsymbol{\Theta}, v(\cdot))$, or a closed-loop control for the initial state $x \in \mathbb{R}^{n}$.

Note that in the above, $A_{\Theta}$ and $C_{\Theta}$ only depend on $\Theta$, and $\bar{A}_{\Theta}$ and $\bar{C}_{\Theta}$ depend on $\Theta=(\Theta, \bar{\Theta})$. Also, one sees that the corresponding coefficients $B, \bar{B}, D, \bar{D}$ of the control process, as well as the nonhomogeneous terms $b(\cdot), \sigma(\cdot)$ are unchanged under $(2.7)$. See [23] for a relevant presentation.

We introduce the following assumption.

(H1) System $[A, \bar{A}, C, \bar{C} ; B, \bar{B}, D, \bar{D}]$ is MF- $L^{2}$-stablizable, i.e., $\mathscr{S}[A, \bar{A}, C, \bar{C} ; B, \bar{B}, D, \bar{D}] \neq \varnothing$.

We have the following result.

Proposition 2.4. Let (H1) hold. Then for any $x \in \mathbb{R}^{n}, \mathscr{U}_{a d}(x) \neq \varnothing$ and $u(\cdot) \in \mathscr{U}_{a d}(x)$ if and only if $u(\cdot)=$ $u^{\Theta, v}(\cdot)$ given by $(2.7)$ for some $\boldsymbol{\Theta} \equiv(\Theta, \bar{\Theta}) \in \mathscr{S}[A, \bar{A}, C, \bar{C} ; B, \bar{B}, D, \bar{D}]$ and $v(\cdot) \in L_{\mathbb{F}}^{2}\left(\mathbb{R}^{m}\right)$, where $X(\cdot) \equiv$ $X^{\Theta, v}(\cdot)$ is the solution to the closed-loop system (2.8).

Proof. Sufficiency. Let $v(\cdot) \in L_{\mathbb{F}}^{2}\left(\mathbb{R}^{m}\right)$ and $X(\cdot)$ be the solution to $(2.8)$. Since system $\left[A_{\Theta}, \bar{A}_{\Theta}, C_{\Theta}, \bar{C}_{\Theta}\right]$ is $L^{2}$ asymptotically stable and system $\left[A_{\Theta}, C_{\Theta}\right]$ is $L^{2}$-globally integrable, by Proposition 2.2, the solution $X(\cdot)$ to $(2.8)$ is in $\mathscr{X}[0, \infty)$. Hence, setting $u(\cdot)$ by $(2.7)$, we see that $u(\cdot) \in L_{\mathbb{F}}^{2}\left(\mathbb{R}^{m}\right)$. By the uniqueness of the solutions, one has that $X(\cdot) \equiv X^{\Theta, v}(\cdot)$ also solves $(2.1)$. Therefore, $u(\cdot) \in \mathscr{U}_{a d}(x)$.

Necessity. Assume that $u(\cdot) \in \mathscr{U}_{a d}(x)$. Let $\Theta \equiv(\Theta, \bar{\Theta}) \in \mathscr{S}[A, \bar{A}, C, \bar{C} ; B, \bar{B}, D, \bar{D}]$ and the corresponding $X(\cdot) \in \mathscr{X}[0, \infty)$ be the solution to $(2.1)$. Set $v(\cdot) \triangleq u(\cdot)-\Theta\{X(\cdot)-\mathbb{E}[X(\cdot)]\}-\bar{\Theta} \mathbb{E}[X(\cdot)] \in L_{\mathbb{F}}^{2}\left(\mathbb{R}^{m}\right)$. By the uniqueness of the solutions again, $X(\cdot)$ coincides with the solution to $(2.8)$. Thus, $u(\cdot)$ admits a representation of the form (2.7). The proof is complete.

From the above, we can easily show that under $(\mathrm{H} 1), \mathscr{U}_{a d}(x)=L_{\mathbb{F}}^{2}\left(\mathbb{R}^{m}\right)$ which is independent of $x$. Hence, hereafter, once (H1) is assumed, we will denote $\mathscr{U}_{a d}(x)=\mathscr{U}_{a d}$. Now, we introduce the following definitions concerning Problem (MF-SLQ).

Definition 2.5. (i) Problem (MF-SLQ) is said to be finite at $x \in \mathbb{R}^{n}$ if $V(x)>-\infty$, and Problem (MF-SLQ) is said to be finite if it is finite at every $x \in \mathbb{R}^{n}$. 
(ii) An element $u^{*}(\cdot) \in \mathscr{U}_{a d}(x)$ is called an open-loop optimal control of Problem (MF-SLQ) for the initial state $x \in \mathbb{R}^{n}$ if

$$
J\left(x ; u^{*}(\cdot)\right) \leqslant J(x ; u(\cdot)), \quad \forall u(\cdot) \in \mathscr{U}_{a d}(x) .
$$

If an open-loop optimal control (uniquely) exists for $x \in \mathbb{R}^{n}$, Problem (MF-SLQ) is said to be (uniquely) openloop solvable at $x$. Problem (MF-SLQ) is said to be (uniquely) open-loop solvable if it is (uniquely) open-loop solvable at all $x \in \mathbb{R}^{n}$.

(iii) A pair $\left(\Theta^{*}, v^{*}(\cdot)\right) \in \mathscr{S}[A, \bar{A}, C, \bar{C} ; B, \bar{B}, D, \bar{D}] \times L_{\mathbb{F}}^{2}\left(\mathbb{R}^{m}\right)$ is called a closed-loop optimal strategy if

$$
\begin{gathered}
J\left(x ; \Theta^{*}\left\{X^{*}(\cdot)-\mathbb{E}\left[X^{*}(\cdot)\right]\right\}+\bar{\Theta}^{*} \mathbb{E}\left[X^{*}(\cdot)\right]+v^{*}(\cdot)\right) \leqslant J(x ; \Theta\{X(\cdot)-\mathbb{E}[X(\cdot)]\}+\bar{\Theta} \mathbb{E}[X(\cdot)]+v(\cdot)), \\
\forall(\Theta, v(\cdot)) \in \mathscr{S}[A, \bar{A}, C, \bar{C} ; B, \bar{B}, D, \bar{D}] \times L_{\mathbb{F}}^{2}\left(\mathbb{R}^{m}\right), x \in \mathbb{R}^{n},
\end{gathered}
$$

where $X^{*}(\cdot) \equiv X^{\boldsymbol{\Theta}^{*}, v^{*}}(\cdot)$ and $X(\cdot) \equiv X^{\boldsymbol{\Theta}, v}(\cdot)$ are the closed-loop state processes corresponding to $\left(x, \boldsymbol{\Theta}^{*}, v^{*}(\cdot)\right)$ and $(x, \boldsymbol{\Theta}, v(\cdot))$, respectively. If an optimal closed-loop strategy (uniquely) exists, Problem (MF-SLQ) is said to be (uniquely) closed-loop solvable.

(iv) An open-loop optimal controls $u^{*}(\cdot ; x) \in \mathscr{U}_{a d}$ of Problem (MF-SLQ), parameterized by $x \in \mathbb{R}^{n}$, admits a closed-loop representation, if there exists a pair $\left(\boldsymbol{\Theta}^{*}, v^{*}(\cdot)\right) \in \mathscr{S}[A, \bar{A}, C, \bar{C} ; B, \bar{B}, D, \bar{D}] \times L_{\mathbb{F}}^{2}\left(\mathbb{R}^{m}\right)$ such that for any initial state $x \in \mathbb{R}^{n}$,

$$
u^{*}(\cdot) \equiv \Theta^{*}\left\{X^{*}(\cdot)-\mathbb{E}\left[X^{*}(\cdot)\right]\right\}+\bar{\Theta}^{*} \mathbb{E}\left[X^{*}(\cdot)\right]+v^{*}(\cdot)
$$

where $X^{*}(\cdot) \equiv X^{\Theta^{*}, v^{*}}(\cdot) \in \mathscr{X}[0, \infty)$ is the solution to the closed-loop system (2.8) corresponding to $\left(\mathbf{\Theta}^{*}, v^{*}(\cdot)\right)$.

Similar to Proposition 2.5 of [31], we have that $\left(\boldsymbol{\Theta}^{*}, v^{*}(\cdot)\right) \in \mathscr{S}[A, \bar{A}, C, \bar{C} ; B, \bar{B}, D, \bar{D}] \times L_{\mathbb{F}}^{2}\left(\mathbb{R}^{m}\right)$ is an optimal closed-loop strategy, if and only if the following condition holds:

$$
J\left(x ; \Theta^{*}\left\{X^{*}(\cdot)-\mathbb{E}\left[X^{*}(\cdot)\right]\right\}+\bar{\Theta}^{*} \mathbb{E}\left[X^{*}(\cdot)\right]+v^{*}(\cdot)\right) \leqslant J\left(x ; \Theta^{*}\{X(\cdot)-\mathbb{E}[X(\cdot)]\}+\bar{\Theta}^{*} \mathbb{E}[X(\cdot)]+v(\cdot)\right),
$$

for any $(x, v(\cdot)) \in \mathbb{R}^{n} \times L_{\mathbb{F}}^{2}\left(\mathbb{R}^{m}\right)$, where $X^{*}(\cdot) \equiv X^{\Theta^{*}, v^{*}}(\cdot)$ and $X(\cdot) \equiv X^{\Theta^{*}, v}(\cdot)$ are the closed-loop state processes corresponding to $\left(x, \boldsymbol{\Theta}^{*}, v^{*}(\cdot)\right)$ and $\left(x, \boldsymbol{\Theta}^{*}, v(\cdot)\right)$, respectively. On the other hand, from Proposition 2.4, we see that under $(\mathrm{H} 1),(2.13)$ is equivalent to the following:

$$
J\left(x ; \Theta^{*}\left\{X^{*}(\cdot)-\mathbb{E}\left[X^{*}(\cdot)\right]\right\}+\bar{\Theta}^{*} \mathbb{E}\left[X^{*}(\cdot)\right]+v^{*}(\cdot)\right) \leqslant J(x ; u(\cdot)), \quad \forall(x, u(\cdot)) \in \mathbb{R}^{n} \times \mathscr{U}_{a d} .
$$

In general, an open-loop optimal control depends on the initial state $x \in \mathbb{R}^{n}$, whereas a closed-loop strategy is required to be independent of $x$. From (2.14), we see that the outcome $u^{*}(\cdot)$ given by (2.12) for some closed-loop strategy $\left(\boldsymbol{\Theta}^{*}, v^{*}(\cdot)\right)$ is an open-loop optimal control for the initial state $X^{*}(0)$. Hence, for Problem (MF-SLQ), the closed-loop solvability implies the open-loop solvability. The converse is also true for stochastic LQ optimal control problems in an infinite horizon without mean fields. That is to say, the open-loop and closed-loop solvabilities are equivalent (see [46]).

It is natural for us to ask: Do we have such equivalence for Problem (MF-SLQ)? To answer this question, we first present the result concerning the characterization of open-loop and closed-loop solvabilities of Problem (MF-SLQ). To simplify notation, in what follows, we denote

$$
\widehat{A}=A+\bar{A}, \quad \widehat{B}=B+\bar{B}, \quad \widehat{C}=C+\bar{C}, \quad \widehat{D}=D+\bar{D}, \quad \widehat{Q}=Q+\bar{Q}, \quad \widehat{S}=S+\bar{S}, \quad \widehat{R}=R+\bar{R} .
$$


Note that for any $\boldsymbol{\Theta} \equiv(\Theta, \bar{\Theta}) \in \mathscr{S}[A, \bar{A}, C, \bar{C} ; B, \bar{B}, D, \bar{D}]$ and $v(\cdot) \in L_{\mathbb{F}}^{2}\left(\mathbb{R}^{m}\right)$, we have the closed-loop system (2.8). The cost functional (2.2) becomes

$$
\begin{aligned}
& J^{\Theta}(x ; v(\cdot)) \equiv J(x ; \Theta(X(\cdot)-\mathbb{E}[X(\cdot)])+\bar{\Theta} \mathbb{E}[X(\cdot)]+v(\cdot)) \\
& =\mathbb{E} \int_{0}^{\infty}\left[\left\langle\left(\begin{array}{cc}
Q_{\Theta} & S_{\Theta}^{\top} \\
S_{\Theta} & R
\end{array}\right)\left(\begin{array}{c}
X \\
v
\end{array}\right),\left(\begin{array}{c}
X \\
v
\end{array}\right)\right\rangle+2\left\langle\left(\begin{array}{c}
q_{\Theta}(t) \\
\rho(t)
\end{array}\right),\left(\begin{array}{c}
X \\
v
\end{array}\right)\right\rangle+\left\langle\left(\begin{array}{cc}
\bar{Q}_{\Theta} & \bar{S}_{\Theta}^{\top} \\
\bar{S}_{\Theta} & \bar{R}
\end{array}\right)\left(\begin{array}{c}
\mathbb{E}[X] \\
\mathbb{E}[v]
\end{array}\right),\left(\begin{array}{c}
\mathbb{E}[X] \\
\mathbb{E}[v]
\end{array}\right)\right\rangle\right] \mathrm{d} t,
\end{aligned}
$$

where

$$
\left\{\begin{array}{l}
Q_{\Theta}=Q+S^{\top} \Theta+\Theta^{\top} S+\Theta^{\top} R \Theta, \quad \bar{Q}_{\Theta}=\bar{Q}+\widehat{S}^{\top} \bar{\Theta}+\bar{\Theta}^{\top} \widehat{S}+\bar{\Theta}^{\top} \widehat{R} \bar{\Theta}-S^{\top} \Theta-\Theta^{\top} S-\Theta^{\top} R \Theta, \\
S_{\Theta}=S+R \Theta, \quad \bar{S}_{\Theta}=\bar{S}+\widehat{R} \bar{\Theta}-R \Theta, \quad q_{\Theta}(\cdot)=q(\cdot)+\Theta^{\top}(\rho(\cdot)-\mathbb{E}[\rho(\cdot)])+\bar{\Theta}^{\top} \mathbb{E}[\rho(\cdot)] .
\end{array}\right.
$$

We see that $Q_{\Theta}, S_{\Theta}$ depend on $\Theta, \bar{Q}_{\Theta}, \bar{S}_{\Theta}, q_{\Theta}$ depend on $\Theta \equiv(\Theta, \bar{\Theta})$, and $R(\cdot), \bar{R}(\cdot), \rho(\cdot)$ are unchanged. Similar to $(2.15)$, we will denote

$$
\widehat{A}_{\Theta}=A_{\Theta}+\bar{A}_{\Theta}, \quad \widehat{C}_{\Theta}=C_{\Theta}+\bar{C}_{\Theta}, \quad \widehat{Q}_{\Theta}=Q_{\Theta}+\bar{Q}_{\Theta}, \quad \widehat{S}_{\Theta}=S_{\Theta}+\bar{S}_{\Theta}
$$

In later investigations, we will encounter the comparison between two closed-loop strategies. Therefore, we need the following definition.

Definition 2.6. Let $(\boldsymbol{\Theta}, v(\cdot)),\left(\Theta^{\prime}, v^{\prime}(\cdot)\right) \in \mathscr{S}[A, \bar{A}, C, \bar{C} ; B, \bar{B}, D, \bar{D}] \times L_{\mathbb{F}}^{2}\left(\mathbb{R}^{n}\right)$.

(i) We say that $(\boldsymbol{\Theta}, v(\cdot))$ and $\left(\boldsymbol{\Theta}^{\prime}, v^{\prime}(\cdot)\right)$ are intrinsically different if for some $x \in \mathbb{R}^{n}, X(\cdot x, \boldsymbol{\Theta}, v(\cdot)) \neq$ $X\left(\cdot ; x, \mathbf{\Theta}^{\prime}, v^{\prime}(\cdot)\right)$.

(ii) We say that $(\boldsymbol{\Theta}, v(\cdot))$ and $\left(\boldsymbol{\Theta}^{\prime}, v^{\prime}(\cdot)\right)$ are intrinsically the same if for any $x \in \mathbb{R}^{n}, X(\cdot x, \boldsymbol{\Theta}, v(\cdot))=$ $X\left(\cdot ; x, \mathbf{\Theta}^{\prime}, v^{\prime}(\cdot)\right)$.

Remark 2.7. The point that we would like to make here is that sometimes, $(\boldsymbol{\Theta}, v(\cdot)) \neq\left(\boldsymbol{\Theta}^{\prime}, v^{\prime}(\cdot)\right)$. But they could be intrinsically the same. Here is such a situation. Let $(\boldsymbol{\Theta}, v(\cdot)) \neq\left(\boldsymbol{\Theta}^{\prime}, v^{\prime}(\cdot)\right)$ and let $X(\cdot)$ and $X^{\prime}(\cdot)$ be the corresponding state processes. Then (note (2.9) and (2.15))

$$
\begin{array}{ll}
A_{\Theta}-A_{\Theta^{\prime}}=B\left(\Theta-\Theta^{\prime}\right), & \bar{A}_{\Theta}-\bar{A}_{\Theta^{\prime}}=\widehat{B}\left(\bar{\Theta}-\bar{\Theta}^{\prime}\right)-B\left(\Theta-\Theta^{\prime}\right), \\
C_{\Theta}-C_{\Theta^{\prime}}=D\left(\Theta-\Theta^{\prime}\right), & \bar{C}_{\Theta}-\bar{C}_{\Theta^{\prime}}=\widehat{D}\left(\bar{\Theta}-\bar{\Theta}^{\prime}\right)-D\left(\Theta-\Theta^{\prime}\right) .
\end{array}
$$

Thus, if

$$
\begin{aligned}
& \mathscr{R}\left(\Theta-\Theta^{\prime}\right) \subseteq \mathscr{N}(B) \cap \mathscr{N}(D), \quad \mathscr{R}\left(\bar{\Theta}-\bar{\Theta}^{\prime}\right) \subseteq \mathscr{N}(\widehat{B}) \cap \mathscr{N}(\widehat{D}) \\
& v(t)-v^{\prime}(t) \in \mathscr{N}(B) \cap \mathscr{N}(D), \quad \mathbb{E}\left[v(t)-v^{\prime}(t)\right] \in \mathscr{N}(\bar{B}) \cap \mathscr{N}(\bar{D}), \quad \forall t \in[0, \infty),
\end{aligned}
$$

then the closed-loop systems under $(\boldsymbol{\Theta}, v(\cdot))$ and $\left(\boldsymbol{\Theta}^{\prime}, v^{\prime}(\cdot)\right)$ are the same. By definition, this means that if $(\boldsymbol{\Theta}, v(\cdot)),\left(\boldsymbol{\Theta}^{\prime}, v^{\prime}(\cdot)\right)$ are two closed-loop strategies such that $(2.19)$ holds, then they are intrinsically the same. If the above fails, then the two closed-loop strategies will be intrinsically different.

Note that there is another issue when we compare two closed-loop strategies, namely, the corresponding costs could be different. But, we prefer to concentrate on the difference of the corresponding state processes, which will be mainly used later. 
Definition 2.8. The following is called a system of generalized AREs:

$$
\left\{\begin{array}{l}
P A+A^{\top} P+C^{\top} P C+Q-\left(P B+C^{\top} P D+S^{\top}\right) \Sigma^{\dagger}\left(B^{\top} P+D^{\top} P C+S\right)=0, \\
\widehat{P} \widehat{A}+\widehat{A}^{\top} \widehat{P}+\widehat{C}^{\top} P \widehat{C}+\widehat{Q}-\left(\widehat{P} \widehat{B}+\widehat{C}^{\top} P \widehat{D}+\widehat{S}^{\top}\right) \bar{\Sigma}^{\dagger}\left(\widehat{B}^{\top} \widehat{P}+\widehat{D}^{\top} P \widehat{C}+\widehat{S}\right)=0, \\
\Sigma \equiv R+D^{\top} P D \geqslant 0, \quad \bar{\Sigma} \equiv \widehat{R}+\widehat{D}^{\top} P \widehat{D} \geqslant 0, \\
\mathscr{R}\left(B^{\top} P+D^{\top} P C+S\right) \subseteq \mathscr{R}(\Sigma), \quad \mathscr{R}\left(\widehat{B}^{\top} \widehat{P}+\widehat{D}^{\top} P \widehat{C}+\widehat{S}\right) \subseteq \mathscr{R}(\bar{\Sigma}),
\end{array}\right.
$$

with the unknown $(P, \widehat{P}) \in \mathbb{S}^{n} \times \mathbb{S}^{n}$. A solution pair $(P, \widehat{P})$ to $(2.20)$ is said to be static stabilizing if there exists a pair $(\theta, \bar{\theta}) \in \mathbb{R}^{m \times 2 n}$, such that $\Theta \equiv(\Theta, \bar{\Theta}) \in \mathscr{S}[A, \bar{A}, C, \bar{C} ; B, \bar{B}, D, \bar{D}]$, where

$$
\Theta=-\Sigma^{\dagger}\left(B^{\top} P+D^{\top} P C+S\right)+\left(I-\Sigma^{\dagger} \Sigma\right) \theta, \quad \bar{\Theta}=-\bar{\Sigma}^{\dagger}\left(\widehat{B}^{\top} \widehat{P}+\widehat{D}^{\top} P \widehat{C}+\widehat{S}\right)+\left(I-\bar{\Sigma}^{\dagger} \bar{\Sigma}\right) \bar{\theta}
$$

We now state the following result, which is an extension of a result without mean-field terms in [46] and will play an important role in the next section. We omit the proof here and refer the interested readers to Li-Shi-Yong [29], an arxiv version of the current paper.

Theorem 2.9. Let (H1) hold. Then the following are equivalent:

(i) Problem (MF-SLQ) is open-loop solvable.

(ii) Problem (MF-SLQ) is closed-loop solvable.

(iii) The system (2.20) admits a static stabilizing solution pair $(P, \widehat{P}) \in \mathbb{S}^{n} \times \mathbb{S}^{n}$, the BSDE on $[0, \infty)$ :

$$
\begin{array}{r}
-\mathrm{d} \eta(t)=\left\{A^{\top} \eta(t)-\left(B^{\top} P+D^{\top} P C+S\right)^{\top} \Sigma^{\dagger}\left[B^{\top} \eta(t)+D^{\top}(\zeta(t)+P \sigma(t))+\rho(t)\right]\right. \\
\left.+C^{\top}[\zeta(t)+P \sigma(t)]+P b(t)+q(t)\right\} \mathrm{d} t-\zeta(t) \mathrm{d} W(t), \quad t \geqslant 0,
\end{array}
$$

admits an adapted solution $(\eta(\cdot), \zeta(\cdot)) \in \mathscr{X}[0, \infty) \times L_{\mathbb{F}}^{2}\left(\mathbb{R}^{n}\right)$ such that

$$
\begin{array}{r}
B^{\top}[\eta(t)-\mathbb{E}[\eta(t)]]+D^{\top}[\zeta(t)-\mathbb{E}[\zeta(t)]]+D^{\top} P[\sigma(t)-\mathbb{E}[\sigma(t)]]+\rho(t)-\mathbb{E}[\rho(t)] \in \mathscr{R}(\Sigma), \\
\text { a.e. } t \in[0, \infty), \text { a.s. }
\end{array}
$$

and the ODE on $[0, \infty)$ :

$$
\begin{aligned}
& \dot{\bar{\eta}}(t)+\widehat{A}^{\top} \bar{\eta}(t)-\left(\widehat{B}^{\top} \widehat{P}+\widehat{D}^{\top} P \widehat{C}+\widehat{S}\right)^{\top} \bar{\Sigma}^{\dagger}\left\{\widehat{B}^{\top} \bar{\eta}(t)+\widehat{D}^{\top} \mathbb{E}[\zeta(t)+P \sigma(t)]+\mathbb{E}[\rho(t)]\right\} \\
& +\widehat{C}^{\top} \mathbb{E}[\zeta(t)+P \sigma(t)]+\mathbb{E}[\widehat{P} b(t)+q(t)]=0,
\end{aligned}
$$

admits a solution $\bar{\eta}(\cdot) \in L^{2}\left(\mathbb{R}^{n}\right)$ such that

$$
\widehat{B}^{\top} \bar{\eta}(t)+\widehat{D}^{\top} \mathbb{E}[\zeta(t)]+\widehat{D}^{\top} P \mathbb{E}[\sigma(t)]+\mathbb{E}[\rho(t)] \in \mathscr{R}(\bar{\Sigma}), \quad \text { a.e. } t \in[0, \infty) .
$$

In the above case, the closed-loop optimal strategy $\left(\Theta^{*}, v^{*}(\cdot)\right) \equiv\left(\Theta^{*}, \bar{\Theta}^{*}, v^{*}(\cdot)\right)$ is given by

$$
\left\{\begin{array}{l}
\Theta^{*}=-\Sigma^{\dagger}\left(B^{\top} P+D^{\top} P C+S\right)+\left(I-\Sigma^{\dagger} \Sigma\right) \theta \\
\bar{\Theta}^{*}=-\bar{\Sigma}^{\dagger}\left(\widehat{B}^{\top} \widehat{P}+\widehat{D}^{\top} P \widehat{C}+\widehat{S}\right)+\left(I-\bar{\Sigma}^{\dagger} \bar{\Sigma}\right) \bar{\theta} \\
v^{*}(\cdot)=\varphi(\cdot)-\mathbb{E}[\varphi(\cdot)]+\bar{\varphi}(\cdot)+\left(I-\Sigma^{\dagger} \Sigma\right)(\nu(\cdot)-\mathbb{E}[\nu(\cdot)])+\left(I-\bar{\Sigma}^{\dagger} \bar{\Sigma}\right) \bar{\nu}(\cdot),
\end{array}\right.
$$


with $\Theta^{*} \equiv\left(\Theta^{*}, \bar{\Theta}^{*}\right) \in \mathscr{S}[A, \bar{A}, C, \bar{C} ; B, \bar{B}, D, \bar{D}]$, for some $\theta, \bar{\theta} \in \mathbb{R}^{m \times n}, \nu(\cdot), \bar{\nu}(\cdot) \in L^{2}\left(\mathbb{R}^{m}\right)$, and

$$
\left\{\begin{array}{l}
\varphi(\cdot) \triangleq-\Sigma^{\dagger}\left\{B^{\top} \eta(\cdot)+D^{\top}[\zeta(\cdot)+P \sigma(\cdot)]+\rho(\cdot)\right\} \\
\bar{\varphi}(\cdot) \triangleq-\bar{\Sigma}^{\dagger}\left\{\widehat{B}^{\top} \bar{\eta}(\cdot)+\widehat{D}^{\top} \mathbb{E}[\zeta(\cdot)+P \sigma(\cdot)]+\mathbb{E}[\rho(\cdot)]\right\}
\end{array}\right.
$$

Every open-loop optimal control $u^{*}(\cdot)$ for the initial state $x \in \mathbb{R}^{n}$ admits a closed-loop representation (2.12), where $X^{*}(\cdot) \in \mathscr{X}[0, \infty)$ is the solution to the closed-loop system $(2.8)$ under $\left(\boldsymbol{\Theta}^{*}, v^{*}(\cdot)\right)$. Further, the value function is given by

$$
\begin{array}{r}
V(x)=\langle\widehat{P} x, x\rangle+2\langle\bar{\eta}(0), x\rangle+\mathbb{E} \int_{0}^{\infty}[\langle P \sigma(t), \sigma(t)\rangle+2\langle\eta(t), b(t)-\mathbb{E}[b(t)]\rangle+2\langle\bar{\eta}(t), \mathbb{E}[b(t)]\rangle \\
+2\langle\zeta(t), \sigma(t)\rangle-\langle\Sigma(\varphi(t)-\mathbb{E}[\varphi(t)]), \varphi(t)-\mathbb{E}[\varphi(t)]\rangle-\langle\bar{\Sigma} \bar{\varphi}(t), \bar{\varphi}(t)\rangle] \mathrm{d} t
\end{array}
$$

\section{MeAN-FIELD LQ NON-ZERo SUM STOCHAStiC DiffERENTiAL GAMES}

We now return to our Problem (MF-SDG).

\subsection{Notions of Nash equilibria}

To simplify the notation, we let $m=m_{1}+m_{2}$ and denote (for $i=1,2$ )

$$
\left\{\begin{array}{l}
B=\left(B_{1}, B_{2}\right), \quad \bar{B}=\left(\bar{B}_{1}, \bar{B}_{2}\right), \quad D=\left(D_{1}, D_{2}\right), \quad \bar{D}=\left(\bar{D}_{1}, \bar{D}_{2}\right), \\
S_{i}=\left(\begin{array}{l}
S_{i 1} \\
S_{i 2}
\end{array}\right), \quad \bar{S}_{i}=\left(\begin{array}{c}
\bar{S}_{i 1} \\
\bar{S}_{i 2}
\end{array}\right), \quad R_{i}=\left(\begin{array}{ll}
R_{i 11} & R_{i 12} \\
R_{i 21} & R_{i 22}
\end{array}\right) \equiv\left(\begin{array}{l}
R_{i 1} \\
R_{i 2}
\end{array}\right), \\
\bar{R}_{i}=\left(\begin{array}{ll}
\bar{R}_{i 11} & \bar{R}_{i 12} \\
\bar{R}_{i 21} & \bar{R}_{i 22}
\end{array}\right) \equiv\left(\begin{array}{l}
\bar{R}_{i 1} \\
\bar{R}_{i 2}
\end{array}\right), \quad q_{i}(\cdot)=\left(\begin{array}{c}
q_{i 1}(\cdot) \\
q_{i 2}(\cdot)
\end{array}\right), \quad \rho_{i}(\cdot)=\left(\begin{array}{c}
\rho_{i 1}(\cdot) \\
\rho_{i 2}(\cdot)
\end{array}\right), \quad u(\cdot)=\left(\begin{array}{c}
u_{1}(\cdot) \\
u_{2}(\cdot)
\end{array}\right) .
\end{array}\right.
$$

Then the state equation (1.1) becomes

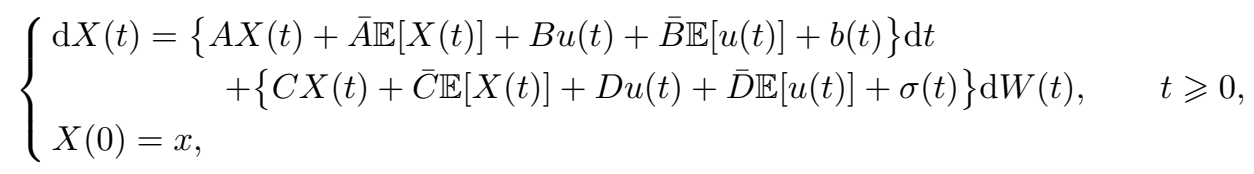

which is of the same form as (2.1), and the cost functionals are, for $i=1,2$,

$$
\begin{gathered}
J_{i}(x ; u(\cdot))=\mathbb{E} \int_{0}^{\infty}\left[\left\langle\begin{array}{cc}
Q_{i} & S_{i}^{\top} \\
S_{i} & R_{i}
\end{array}\right)\left(\begin{array}{c}
X(t) \\
u(t)
\end{array}\right),\left(\begin{array}{c}
X(t) \\
u(t)
\end{array}\right)\right\rangle+2\left\langle\left(\begin{array}{c}
q_{i}(t) \\
\rho_{i}(t)
\end{array}\right),\left(\begin{array}{c}
X(t) \\
u(t)
\end{array}\right)\right\rangle \\
\left.+\left\langle\left(\begin{array}{cc}
\bar{Q}_{i} & \bar{S}_{i}^{\top} \\
\bar{S}_{i} & \bar{R}_{i}
\end{array}\right)\left(\begin{array}{c}
\mathbb{E}[X(t)] \\
\mathbb{E}[u(t)]
\end{array}\right),\left(\begin{array}{c}
\mathbb{E}[X(t)] \\
\mathbb{E}[u(t)]
\end{array}\right)\right\rangle\right] \mathrm{d} t
\end{gathered}
$$

In order the game to make sense, we make a convention that both players at least want to keep the state $X(\cdot) \equiv X\left(\cdot ; x, u_{1}(\cdot), u_{2}(\cdot)\right)$ in $\mathscr{X}[0, \infty)$ so that both cost functionals are well-defined. To guarantee this, similar to Problem (MF-SLQ), we introduce the following assumption.

(H2) System $[A, \bar{A}, C, \bar{C} ; B, \bar{B}, D, \bar{D}]$ is MF- $L^{2}$-stablizable, i.e., $\mathscr{S}[A, \bar{A}, C, \bar{C} ; B, \bar{B}, D, \bar{D}] \neq \varnothing$.

Although (H2) looks the same as (H1), the meaning is different. Hypothesis (H2) provides the possibility for both players to make both cost functionals finite cooperatively. In fact, under (H2), for any $x \in \mathbb{R}^{n}$, by 
Proposition 2.4, the following set of all admissible control pairs is non-empty:

$$
\mathscr{U}_{a d}(x)=\left\{u(\cdot)=\left(u_{1}(\cdot), u_{2}(\cdot)\right) \in L_{\mathbb{F}}^{2}\left(\mathbb{R}^{m}\right) \mid X(\cdot) \equiv X(\cdot ; x, u(\cdot)) \in \mathscr{X}[0, \infty)\right\}
$$

Further, for any $u_{2}(\cdot) \in L_{\mathbb{F}}^{2}\left(\mathbb{R}^{m_{2}}\right)$, making use of Proposition 2.4 again, the following holds:

$$
\mathscr{U}_{a d}^{1}(x)=\left\{u_{1}(\cdot) \in L_{\mathbb{F}}^{2}\left(\mathbb{R}^{m_{1}}\right) \mid \exists u_{2}(\cdot) \in L_{\mathbb{F}}^{2}\left(\mathbb{R}^{m_{2}}\right),\left(u_{1}(\cdot), u_{2}(\cdot)\right) \in \mathscr{U}_{a d}(x)\right\} \neq \varnothing
$$

Likewise, for any $u_{1}(\cdot) \in L_{\mathbb{F}}^{2}\left(\mathbb{R}^{m_{1}}\right)$, one has

$$
\mathscr{U}_{a d}^{2}(x)=\left\{u_{2}(\cdot) \in L_{\mathbb{F}}^{2}\left(\mathbb{R}^{m_{2}}\right) \mid \exists u_{1}(\cdot) \in L_{\mathbb{F}}^{2}\left(\mathbb{R}^{m_{1}}\right),\left(u_{1}(\cdot), u_{2}(\cdot)\right) \in \mathscr{U}_{a d}(x)\right\} \neq \varnothing
$$

We now present the following definition.

Definition 3.1. A $u^{*}(\cdot) \equiv\left(u_{1}^{*}(\cdot), u_{2}^{*}(\cdot)\right) \in \mathscr{U}_{a d}(x)$ is called an open-loop Nash equilibrium of Problem (MF-SDG) for the initial state $x \in \mathbb{R}^{n}$ if

$$
\begin{array}{ll}
J_{1}\left(x ; u_{1}^{*}(\cdot), u_{2}^{*}(\cdot)\right) \leqslant J_{1}\left(x ; u_{1}(\cdot), u_{2}^{*}(\cdot)\right), & \forall u_{1}(\cdot) \in \mathscr{U}_{a d}^{1}(x), \\
J_{2}\left(x ; u_{1}^{*}(\cdot), u_{2}^{*}(\cdot)\right) \leqslant J_{2}\left(x ; u_{1}^{*}(\cdot), u_{2}(\cdot)\right), & \forall u_{2}(\cdot) \in \mathscr{U}_{a d}^{2}(x) .
\end{array}
$$

For $\boldsymbol{\Theta}_{i} \equiv\left(\Theta_{i}, \bar{\Theta}_{i}\right) \in \mathbb{R}^{m_{i} \times 2 n}, i=1,2$, we denote $\boldsymbol{\Theta} \equiv\left(\begin{array}{c}\boldsymbol{\Theta}_{1} \\ \boldsymbol{\Theta}_{2}\end{array}\right) \equiv\left(\begin{array}{cc}\Theta_{1} & \bar{\Theta}_{1} \\ \Theta_{2} & \bar{\Theta}_{2}\end{array}\right) \in \mathbb{R}^{m \times 2 n}$, and let

$$
\begin{aligned}
& \mathscr{S}^{1}\left(\boldsymbol{\Theta}_{2}\right) \triangleq\left\{\Theta_{1} \in \mathbb{R}^{2 m_{1} \times n} \mid \boldsymbol{\Theta} \in \mathscr{S}[A, \bar{A}, C, \bar{C} ; B, \bar{B}, D, \bar{D}]\right\}, \\
& \mathscr{S}^{2}\left(\boldsymbol{\Theta}_{1}\right) \triangleq\left\{\boldsymbol{\Theta}_{2} \in \mathbb{R}^{2 m_{2} \times n} \mid \boldsymbol{\Theta} \in \mathscr{S}[A, \bar{A}, C, \bar{C} ; B, \bar{B}, D, \bar{D}]\right\} .
\end{aligned}
$$

It is clear that if $\Theta \in \mathscr{S}[A, \bar{A}, C, \bar{C} ; B, \bar{B}, D, \bar{D}]$, both $\mathscr{S}^{1}\left(\boldsymbol{\Theta}_{2}\right)$ and $\mathscr{S}^{2}\left(\Theta_{1}\right)$ are nonempty. Similar to the optimal control problem case, any $(\boldsymbol{\Theta}, v(\cdot)) \in \mathscr{S}[A, \bar{A}, C, \bar{C} ; B, \bar{B}, D, \bar{D}] \times L_{\mathbb{F}}^{2}\left(\mathbb{R}^{m}\right)$ is called a closedloop strategy of Problem (MF-SDG). For any initial state $x \in \mathbb{R}^{n}$ and closed-loop strategy $(\boldsymbol{\Theta}, v(\cdot)) \in$ $\mathscr{S}[A, \bar{A}, C, \bar{C} ; B, \bar{B}, D, \bar{D}] \times L_{\mathbb{F}}^{2}\left(\mathbb{R}^{m}\right)$, we consider the following linear MF-SDE on $[0, \infty)($ recall $(2.9))$ :

$$
\left\{\begin{aligned}
\mathrm{d} X(t)= & \left\{A_{\Theta} X(t)+\bar{A}_{\Theta} \mathbb{E}[X(t)]+B v(t)+\bar{B} \mathbb{E}[v(t)]+b(t)\right\} \mathrm{d} t \\
& +\left\{C_{\Theta} X(t)+\bar{C}_{\Theta} \mathbb{E}[X(t)]+D v(t)+\bar{D} \mathbb{E}[v(t)]+\sigma(t)\right\} \mathrm{d} W(t), \quad t \geqslant 0 \\
X(0)= & x
\end{aligned}\right.
$$

By Proposition 2.2, (3.8) admits a unique solution $X(\cdot) \in \mathscr{X}[0, \infty)$. If we denote

$$
u_{i}(\cdot)=\Theta_{i}\{X(\cdot)-\mathbb{E}[X(\cdot)]\}+\bar{\Theta}_{i} \mathbb{E}[X(\cdot)]+v_{i}(\cdot), \quad i=1,2,
$$

then (3.8) coincides with the original state equation (3.2). We call $\left(\boldsymbol{\Theta}_{i}, v_{i}(\cdot)\right)$ a closed-loop strategy of Player $i$, and call $(3.8)$ the closed-loop system of the original system under closed-loop strategy $(\boldsymbol{\Theta}, v(\cdot))$. Also, we call $u(\cdot) \equiv\left(u_{1}(\cdot), u_{2}(\cdot)\right)$, with $u_{i}(\cdot)$ defined by $(3.9)$, the outcome of the closed-loop strategy $(\boldsymbol{\Theta}, v(\cdot))$.

With the solution $X(\cdot) \in \mathscr{X}[0, \infty)$ to $(3.8)$, we denote, for $i=1,2$,

$$
\begin{aligned}
& J_{i}(x ; \boldsymbol{\Theta}, v(\cdot)) \equiv J_{i}\left(x ; \boldsymbol{\Theta}_{1}, v_{1}(\cdot) ; \boldsymbol{\Theta}_{2}, v_{2}(\cdot)\right) \equiv J_{i}(x ; \Theta\{X(\cdot)-\mathbb{E}[X(\cdot)]\}+\bar{\Theta} \mathbb{E}[X(\cdot)]+v(\cdot)) \\
& \equiv J_{i}\left(x ; \Theta_{1}\{X(\cdot)-\mathbb{E}[X(\cdot)]\}+\bar{\Theta}_{1} \mathbb{E}[X(\cdot)]+v_{1}(\cdot) ; \Theta_{2}\{X(\cdot)-\mathbb{E}[X(\cdot)]\}+\bar{\Theta}_{2} \mathbb{E}[X(\cdot)]+v_{2}(\cdot)\right)
\end{aligned}
$$


Similarly, we can define

$$
\begin{aligned}
& J_{i}\left(x ; \boldsymbol{\Theta}_{1}, v_{1}(\cdot) ; u_{2}(\cdot)\right) \equiv J_{i}\left(x ; \Theta_{1}\{X(\cdot)-\mathbb{E}[X(\cdot)]\}+\bar{\Theta}_{1} \mathbb{E}[X(\cdot)]+v_{1}(\cdot) ; u_{2}(\cdot)\right), \quad i=1,2 . \\
& J_{i}\left(x ; u_{1}(\cdot) ; \boldsymbol{\Theta}_{2}, v_{2}(\cdot)\right) \equiv J_{i}\left(x ; u_{1}(\cdot) ; \Theta_{2}\{X(\cdot)-\mathbb{E}[X(\cdot)]\}+\bar{\Theta}_{2} \mathbb{E}[X(\cdot)]+v_{2}(\cdot)\right),
\end{aligned}
$$

We now introduce the following definition.

Definition 3.2. A closed-loop strategy $\left(\Theta^{*}, v^{*}(\cdot)\right) \in \mathscr{S}[A, \bar{A}, C, \bar{C} ; B, \bar{B}, D, \bar{D}] \times L_{\mathbb{F}}^{2}\left(\mathbb{R}^{m}\right)$ is called a closedloop Nash equilibrium of Problem (MF-SDG) if for any $\boldsymbol{\Theta}_{1} \in \mathscr{S}^{1}\left(\mathbf{\Theta}_{2}^{*}\right), \boldsymbol{\Theta}_{2} \in \mathscr{S}^{2}\left(\boldsymbol{\Theta}_{1}^{*}\right), v_{1}(\cdot) \in L_{\mathbb{F}}^{2}\left(\mathbb{R}^{m_{1}}\right)$ and $v_{2}(\cdot) \in L_{\mathbb{F}}^{2}\left(\mathbb{R}^{m_{2}}\right)$,

$$
\begin{cases}J_{1}\left(x ; \boldsymbol{\Theta}^{*}, v^{*}(\cdot)\right) \leqslant J_{1}\left(x ; \boldsymbol{\Theta}_{1}, v_{1}(\cdot) ; \boldsymbol{\Theta}_{2}^{*}, v_{2}^{*}(\cdot)\right), & \forall x \in \mathbb{R}^{n}, \\ J_{2}\left(x ; \boldsymbol{\Theta}^{*}, v^{*}(\cdot)\right) \leqslant J_{2}\left(x ; \boldsymbol{\Theta}_{1}^{*}, v_{1}^{*}(\cdot) ; \boldsymbol{\Theta}_{2}, v_{2}(\cdot)\right), & \forall x \in \mathbb{R}^{n} .\end{cases}
$$

Note that on the left-hand sides of (3.10), the involved state is $X(\cdot) \equiv X\left(\cdot ; x, \Theta^{*}, v^{*}(\cdot)\right)$, depending on $\left(\boldsymbol{\Theta}^{*}, v^{*}(\cdot)\right)$. Whereas, on the right-hand sides of $(3.10)$, the involved states are $X(\cdot) \equiv X\left(\cdot ; x, \boldsymbol{\Theta}_{1}, v_{1}(\cdot) ; \boldsymbol{\Theta}_{2}^{*}, v_{2}^{*}(\cdot)\right)$ and $X(\cdot)=X\left(\cdot ; \boldsymbol{\Theta}_{1}^{*}, v_{1}^{*}(\cdot) ; \boldsymbol{\Theta}_{2}, v_{2}(\cdot)\right)$ respectively, which are different in general. We emphasize that the openloop Nash equilibrium $\left(u_{1}^{*}(\cdot), u_{2}^{*}(\cdot)\right)$ usually depends on the initial state $x$, whereas a closed-loop Nash equilibrium $\left(\mathbf{\Theta}^{*}, v^{*}(\cdot)\right)$ is required to be independent of $x$. It is easy to see that $\left(\mathbf{\Theta}^{*}, v^{*}(\cdot)\right)$ is a closed-loop Nash equilibrium of Problem (MF-SDG) if and only if one of the following hold:

(i) For any $v_{1}(\cdot) \in L_{\mathbb{F}}^{2}\left(\mathbb{R}^{m_{1}}\right)$ and $v_{2}(\cdot) \in L_{\mathbb{F}}^{2}\left(\mathbb{R}^{m_{2}}\right)$,

$$
J_{1}\left(x ; \boldsymbol{\Theta}^{*}, v^{*}(\cdot)\right) \leqslant J_{1}\left(x ; \boldsymbol{\Theta}^{*}, v_{1}(\cdot), v_{2}^{*}(\cdot)\right), \quad J_{2}\left(x ; \boldsymbol{\Theta}^{*}, v^{*}(\cdot)\right) \leqslant J_{2}\left(x ; \boldsymbol{\Theta}^{*}, v_{1}^{*}(\cdot), v_{2}(\cdot)\right) ;
$$

(ii) For any $u_{1}(\cdot) \in L_{\mathbb{F}}^{2}\left(\mathbb{R}^{m_{1}}\right)$ and $u_{2}(\cdot) \in L_{\mathbb{F}}^{2}\left(\mathbb{R}^{m_{2}}\right)$,

$$
J_{1}\left(x ; \boldsymbol{\Theta}^{*}, v^{*}(\cdot)\right) \leqslant J_{1}\left(x ; u_{1}(\cdot) ; \boldsymbol{\Theta}_{2}^{*}, v_{2}^{*}(\cdot)\right), \quad J_{2}\left(x ; \boldsymbol{\Theta}^{*}, v^{*}(\cdot)\right) \leqslant J_{2}\left(x ; \boldsymbol{\Theta}_{1}^{*}, v_{1}^{*}(\cdot) ; u_{2}(\cdot)\right) .
$$

If we denote (comparing with (3.10))

$$
u_{i}^{*}(\cdot)=\Theta_{i}^{*}\left\{X^{*}(\cdot)-\mathbb{E}\left[X^{*}(\cdot)\right]\right\}+\bar{\Theta}_{i}^{*} \mathbb{E}\left[X^{*}(\cdot)\right]+v_{i}^{*}(\cdot), \quad i=1,2,
$$

then (3.12) becomes

$$
\begin{aligned}
& J_{1}\left(x ; u_{1}^{*}(\cdot) ; u_{2}^{*}(\cdot)\right) \leqslant J_{1}\left(x ; u_{1}(\cdot) ; \Theta_{2}^{*}\left\{X^{u_{1}, v_{2}^{*}}(\cdot)-\mathbb{E}\left[X^{u_{1}, v_{2}^{*}}(\cdot)\right]\right\}+\bar{\Theta}_{2}^{*} \mathbb{E}\left[X^{u_{1}, v_{2}^{*}}(\cdot)\right]+v_{2}^{*}(\cdot)\right), \\
& J_{2}\left(x ; u_{1}^{*}(\cdot) ; u_{2}^{*}(\cdot)\right) \leqslant J_{2}\left(x ; \Theta_{1}^{*}\left\{X^{v_{1}^{*}, u_{2}}(\cdot)-\mathbb{E}\left[X^{v_{1}^{*}, u_{2}}(\cdot)\right]\right\}+\bar{\Theta}_{1}^{*} \mathbb{E}\left[X^{v_{1}^{*}, u_{2}}(\cdot)\right]+v_{1}^{*}(\cdot) ; u_{2}(\cdot)\right),
\end{aligned}
$$

where $X^{u_{1}, v_{2}^{*}(\cdot)}=X\left(\cdot ; x, u_{1}(\cdot) ; \boldsymbol{\Theta}_{2}^{*}, v_{2}^{*}(\cdot)\right)$ and $X^{v_{1}^{*}, u_{2}}(\cdot)=X\left(\cdot ; x, \boldsymbol{\Theta}_{1}^{*}, v_{1}^{*}(\cdot) ; u_{2}(\cdot)\right)$. Clearly, neither of the following holds in general:

$$
\begin{aligned}
& u_{1}^{*}(\cdot)=\Theta_{1}^{*}\left\{X^{v_{1}^{*}, u_{2}}(\cdot)-\mathbb{E}\left[X^{v_{1}^{*}, u_{2}}(\cdot)\right]\right\}+\bar{\Theta}_{1}^{*} \mathbb{E}\left[X^{v_{1}^{*}, u_{2}}(\cdot)\right]+v_{1}^{*}(\cdot), \\
& u_{2}^{*}(\cdot)=\Theta_{2}^{*}\left\{X^{u_{1}, v_{2}^{*}}(\cdot)-\mathbb{E}\left[X^{u_{1}, v_{2}^{*}}(\cdot)\right]\right\}+\bar{\Theta}_{2}^{*} \mathbb{E}\left[X^{u_{1}, v_{2}^{*}}(\cdot)\right]+v_{2}^{*}(\cdot) .
\end{aligned}
$$

Hence, comparing this with (3.7), we see that the outcome $\left(u_{1}^{*}(\cdot), u_{2}^{*}(\cdot)\right)$ of the closed-loop Nash equilibrium $\left(\Theta^{*}, v^{*}(\cdot)\right)$ given by $(3.25)$ is not necessarily an open-loop Nash equilibrium of Problem (MF-SDG) for $X^{*}(0)=$ $x$. 
On the other hand, if $\left(\mathbf{\Theta}^{*}, v^{*}(\cdot)\right)$ is a closed-loop Nash equilibrium of Problem (MF-SDG), we may consider the following stabilized state equation (recall (2.9)):

$$
\left\{\begin{aligned}
\mathrm{d} X^{v_{1}, v_{2}}(t)= & \left\{A_{\Theta^{*}} X^{v_{1}, v_{2}}(t)+\bar{A}_{\Theta^{*}} \mathbb{E}\left[X^{v_{1}, v_{2}}(t)\right]+B v(t)+\bar{B} \mathbb{E}[v(t)]+b(t)\right\} \mathrm{d} t \\
& +\left\{C_{\Theta^{*}} X^{v_{1}, v_{2}}(t)+\bar{C}_{\Theta^{*}} \mathbb{E}\left[X^{v_{1}, v_{2}}(t)\right]+D v(t)+\bar{D} \mathbb{E}[v(t)]+\sigma(t)\right\} \mathrm{d} W(t), \quad t \geqslant 0 \\
X^{v_{1}, v_{2}}(0)= & x
\end{aligned}\right.
$$

with cost functionals

$$
\begin{aligned}
J_{i}^{\Theta^{*}}\left(x ; v_{1}(\cdot), v_{2}(\cdot)\right) \triangleq J_{i}\left(x ; \Theta_{1}^{*}\left\{X^{v_{1}, v_{2}}(\cdot)-\mathbb{E}\left[X^{v_{1}, v_{2}}(\cdot)\right]\right\}+\bar{\Theta}_{1}^{*} \mathbb{E}\left[X^{v_{1}, v_{2}}(\cdot)\right]+v_{1}(\cdot) ;\right. \\
\left.\Theta_{2}^{*}\left\{X^{v_{1}, v_{2}}(\cdot)-\mathbb{E}\left[X^{v_{1}, v_{2}}(\cdot)\right]\right\}+\bar{\Theta}_{2}^{*} \mathbb{E}\left[X^{v_{1}, v_{2}}(\cdot)\right]+v_{2}(\cdot)\right), \quad i=1,2 .
\end{aligned}
$$

Then by $(3.11)$, it is easy to see that $\left(v_{1}^{*}(\cdot), v_{2}^{*}(\cdot)\right)$ is an open-loop Nash equilibrium of the corresponding mean-field LQ two-person non-zero sum stochastic differential games.

From the above, we see that Problems (MF-SDG) and (MF-SLQ) are essentially different in a certain sense, and we can only say that Problem (MF-SLQ) is formally a special case of Problem (MF-SDG).

\subsection{Open-loop Nash equalibria and their closed-loop representation}

In this section, we discuss the open-loop Nash equilibria for Problem (MF-SDG) in terms of MF-FBSDEs. We first have the following result, whose proof is similar to that of Theorem 4.1 of [47]. Thus, we omit the proof here and refer the interested readers to [29] for a detailed proof.

Theorem 3.3. Let (H2) hold, and $x \in \mathbb{R}^{n}$. Then $u^{*}(\cdot) \equiv\left(u_{1}^{*}(\cdot), u_{2}^{*}(\cdot)\right) \in \mathscr{U}_{a d}(x)$ is an open-loop Nash equilibrium of Problem (MF-SDG) for $x$ if and only if the following two conditions hold:

(i) The adapted solution $\left(X^{*}(\cdot), Y_{i}^{*}(\cdot), Z_{i}^{*}(\cdot)\right) \in \mathscr{X}[0, \infty) \times \mathscr{X}[0, \infty) \times L_{\mathbb{F}}^{2}\left(\mathbb{R}^{n}\right)$ to the following MF-FBSDE:

$$
\left\{\begin{aligned}
& \mathrm{d} X^{*}(t)=\left\{A X^{*}(t)+\bar{A} \mathbb{E}\left[X^{*}(t)\right]+B u^{*}(t)+\bar{B} \mathbb{E}\left[u^{*}(t)\right]+b(t)\right\} \mathrm{d} t \\
&+\left\{C X^{*}(t)+\bar{C} \mathbb{E}\left[X^{*}(t)\right]+D u^{*}(t)+\bar{D} \mathbb{E}\left[u^{*}(t)\right]+\sigma(t)\right\} \mathrm{d} W(t), \\
&-\mathrm{d} Y_{i}^{*}(t)=\left\{\begin{array}{l}
A^{\top} Y_{i}^{*}(t)+\bar{A}^{\top} \mathbb{E}\left[Y_{i}^{*}(t)\right]+C^{\top} Z_{i}^{*}(t)+\bar{C}^{\top} \mathbb{E}\left[Z_{i}^{*}(t)\right]+Q_{i} X^{*}(t)+\bar{Q}_{i} \mathbb{E}\left[X^{*}(t)\right] \\
\left.\quad+S_{i}^{\top} u^{*}(t)+\bar{S}_{i}^{\top} \mathbb{E}\left[u^{*}(t)\right]+q_{i}(t)\right\} \mathrm{d} t-Z_{i}(t) \mathrm{d} W(t), \quad t \geqslant 0, \quad i=1,2,
\end{array}\right. \\
& \\
& X^{*}(0)=x, \quad
\end{aligned}\right.
$$

satisfies the following stationarity condition:

$$
\begin{aligned}
& B_{i}^{\top} Y_{i}^{*}(t)+\bar{B}_{i}^{\top} \mathbb{E}\left[Y_{i}^{*}(t)\right]+D_{i}^{\top} Z_{i}^{*}(t)+\bar{D}_{i}^{\top} \mathbb{E}\left[Z_{i}^{*}(t)\right]+S_{i i} X^{*}(t)+\bar{S}_{i i} \mathbb{E}\left[X^{*}(t)\right] \\
& \quad+R_{i i} u^{*}(t)+\bar{R}_{i i} \mathbb{E}\left[u^{*}(t)\right]+\rho_{i i}(t)=0, \quad \text { a.e. } t \in[0, \infty), \text { a.s., } \quad i=1,2 .
\end{aligned}
$$

(ii) The maps $u_{1}(\cdot) \mapsto J_{1}\left(x ; u_{1}(\cdot), u_{2}(\cdot)\right)$ and $u_{2}(\cdot) \mapsto J_{2}\left(x ; u_{1}(\cdot), u_{2}(\cdot)\right)$ are convex, i.e.,

$$
\begin{aligned}
\mathbb{E} \int_{0}^{\infty} & {\left[\left\langle Q_{i} X_{i}^{0}(t), X_{i}^{0}(t)\right\rangle+2\left\langle S_{i i} X_{i}^{0}(t), u_{i}(t)\right\rangle+\left\langle R_{i i i} u_{i}(t), u_{i}(t)\right\rangle+\left\langle\bar{Q}_{i} \mathbb{E}\left[X_{i}^{0}(t)\right], \mathbb{E}\left[X_{i}^{0}(t)\right]\right\rangle\right.} \\
+ & \left.2\left\langle\bar{S}_{i i} \mathbb{E}\left[X_{i}^{0}(t)\right], \mathbb{E}\left[u_{i}(t)\right]\right\rangle+\left\langle\bar{R}_{i i i} \mathbb{E}\left[u_{i}(t)\right], \mathbb{E}\left[u_{i}(t)\right]\right\rangle\right] \mathrm{d} t \geqslant 0, \quad \forall u(\cdot) \equiv\left(u_{1}(\cdot), u_{2}(\cdot)\right) \in \mathscr{U}_{a d}(x),
\end{aligned}
$$


where $X_{i}^{0}(\cdot) \in \mathscr{X}[0, \infty)$ is the solution to the following homogeneous controlled MF-SDE:

$$
\left\{\begin{aligned}
& \mathrm{d} X_{i}^{0}(t)=\left\{A X_{i}^{0}(t)+\bar{A} \mathbb{E}\left[X_{i}^{0}(t)\right]+B_{i} u_{i}(t)+\bar{B}_{i} \mathbb{E}\left[u_{i}(t)\right]\right\} \mathrm{d} t \\
&+\left\{C X_{i}^{0}(t)+\bar{C} \mathbb{E}\left[X_{i}^{0}(t)\right]+D_{i} u_{i}(t)+\bar{D}_{i} \mathbb{E}\left[u_{i}(t)\right]\right\} \mathrm{d} W(t), \quad t \geqslant 0 \\
& X_{i}^{0}(0)=0, \quad i=1,2
\end{aligned}\right.
$$

Note that (3.17) is a system of coupled MF-FBSDEs, with one forward equation and two backward equations, for which the coupling is through the relation (3.18). Our next task is to investigate the solvability of (3.17)(3.18). To this end, we introduce the following notation (recall (3.1)):

$$
\begin{aligned}
& \mathbf{A}=\left(\begin{array}{cc}
A & 0 \\
0 & A
\end{array}\right), \overline{\mathbf{A}}=\left(\begin{array}{cc}
\bar{A} & 0 \\
0 & \bar{A}
\end{array}\right), \mathbf{C}=\left(\begin{array}{cc}
C & 0 \\
0 & C
\end{array}\right), \overline{\mathbf{C}}=\left(\begin{array}{cc}
\bar{C} & 0 \\
0 & \bar{C}
\end{array}\right) \in \mathbb{R}^{2 n \times 2 n} ; \\
& \mathbf{B}=\left(\begin{array}{cc}
B & 0 \\
0 & B
\end{array}\right), \overline{\mathbf{B}}=\left(\begin{array}{cc}
\bar{B} & 0 \\
0 & \bar{B}
\end{array}\right), \mathbf{D}=\left(\begin{array}{cc}
D & 0 \\
0 & D
\end{array}\right), \overline{\mathbf{D}}=\left(\begin{array}{cc}
\bar{D} & 0 \\
0 & \bar{D}
\end{array}\right) \in \mathbb{R}^{2 n \times 2 m} ; \\
& \mathbf{Q}=\left(\begin{array}{cc}
Q_{1} & 0 \\
0 & Q_{2}
\end{array}\right), \overline{\mathbf{Q}}=\left(\begin{array}{cc}
\bar{Q}_{1} & 0 \\
0 & \bar{Q}_{2}
\end{array}\right) \in \mathbb{S}^{2 n} ; \quad \mathbf{S}=\left(\begin{array}{cc}
S_{1} & 0 \\
0 & S_{2}
\end{array}\right), \overline{\mathbf{S}}=\left(\begin{array}{cc}
\bar{S}_{1} & 0 \\
0 & \bar{S}_{2}
\end{array}\right) \in \mathbb{R}^{2 m \times 2 n} ; \\
& \mathbf{R}=\left(\begin{array}{cc}
R_{1} & 0 \\
0 & R_{2}
\end{array}\right), \overline{\mathbf{R}}=\left(\begin{array}{cc}
\bar{R}_{1} & 0 \\
0 & \bar{R}_{2}
\end{array}\right) \in \mathbb{S}^{2 m} ; q(\cdot)=\left(\begin{array}{c}
q_{1}(\cdot) \\
q_{2}(\cdot)
\end{array}\right) \in L_{\mathbb{F}}^{2}\left(\mathbb{R}^{2 n}\right) ; \rho(\cdot)=\left(\begin{array}{c}
\rho_{1}(\cdot) \\
\rho_{2}(\cdot)
\end{array}\right) \in L_{\mathbb{F}}^{2}\left(\mathbb{R}^{2 m}\right) .
\end{aligned}
$$

With the above notation, MF-FBSDEs (3.17) can be rewritten as

$$
\left\{\begin{aligned}
& \mathrm{d} X^{*}(t)=\left\{A X^{*}+\bar{A} \mathbb{E}\left[X^{*}\right]+B u^{*}+\bar{B} \mathbb{E}\left[u^{*}\right]+b\right\} \mathrm{d} t \\
&+\left\{C X^{*}+\bar{C} \mathbb{E}\left[X^{*}\right]+D u^{*}+\bar{D} \mathbb{E}\left[u^{*}\right]+\sigma\right\} \mathrm{d} W(t), \\
&-d \mathbf{Y}^{*}(t)=\left\{\begin{array}{|l}
\mathbf{A}^{\top} \mathbf{Y}^{*}+\overline{\mathbf{A}}^{\top} \mathbb{E}\left[\mathbf{Y}^{*}\right]+\mathbf{C}^{\top} \mathbf{Z}^{*}+\overline{\mathbf{C}}^{\top} \mathbb{E}\left[\mathbf{Z}^{*}\right]+\mathbf{Q} \mathbf{I}_{n} X^{*}+\overline{\mathbf{Q}} \mathbf{I}_{n} \mathbb{E}\left[X^{*}\right] \\
\left.\quad+\mathbf{S}^{\top} \mathbf{I}_{m} u^{*}+\overline{\mathbf{S}}^{\top} \mathbf{I}_{m} \mathbb{E}\left[u^{*}\right]+q\right\} \mathrm{d} t-\mathbf{Z}^{*} \mathrm{~d} W(t), \quad t \geqslant 0
\end{array}\right. \\
& X^{*}(0)=x, \quad
\end{aligned}\right.
$$

and the stationarity condition (3.18) can be written as

$$
\begin{gathered}
\mathbf{J}^{\top}\left\{\mathbf{B}^{\top} \mathbf{Y}^{*}+\overline{\mathbf{B}}^{\top} \mathbb{E}\left[\mathbf{Y}^{*}\right]+\mathbf{D}^{\top} \mathbf{Z}^{*}+\overline{\mathbf{D}}^{\top} \mathbb{E}\left[\mathbf{Z}^{*}\right]+\mathbf{S I}_{n} X^{*}+\overline{\mathbf{S}} \mathbf{I}_{n} \mathbb{E}\left[X^{*}\right]+\mathbf{R} \mathbf{I}_{m} u^{*}+\overline{\mathbf{R}} \mathbf{I}_{m} \mathbb{E}\left[u^{*}\right]+\rho\right\}=0, \\
\text { a.e. } t \in[0, \infty), \text { a.s. }
\end{gathered}
$$

where

$$
\left\{\begin{array}{l}
\mathbf{Y}^{*}(\cdot)=\left(\begin{array}{c}
Y_{1}^{*}(\cdot) \\
Y_{2}^{*}(\cdot)
\end{array}\right) \in \mathscr{X}[0, \infty)^{2}, \quad \mathbf{Z}^{*}(\cdot)=\left(\begin{array}{c}
Z_{1}^{*}(\cdot) \\
Z_{2}^{*}(\cdot)
\end{array}\right) \in L_{\mathbb{F}}^{2}\left(\mathbb{R}^{2 n}\right), \quad \mathbf{I}_{n}=\left(\begin{array}{c}
I_{n \times n} \\
I_{n \times n}
\end{array}\right) \in \mathbb{R}^{2 n \times n}, \\
\mathbf{I}_{m}=\left(\begin{array}{l}
I_{m \times m} \\
I_{m \times m}
\end{array}\right) \in \mathbb{R}^{2 m \times m}, \quad \mathbf{J}=\left(\begin{array}{cc}
I_{m_{1} \times m_{1}} & 0 \\
0 & 0 \\
0 & 0 \\
0 & I_{m_{2} \times m_{2}}
\end{array}\right)=\left(\begin{array}{ll}
I_{m_{1} \times m_{1}} & 0_{m_{1} \times m_{2}} \\
0_{m_{2} \times m_{1}} & 0_{m_{2} \times m_{2}} \\
0_{m_{1} \times m_{1}} & 0_{m_{1} \times m_{2}} \\
0_{m_{2} \times m_{1}} & I_{m_{2} \times m_{2}}
\end{array}\right) \in \mathbb{R}^{2 m \times m} .
\end{array}\right.
$$

Now, inspired by $[47,53]$, we may obtain a closed-loop representation of open-loop Nash equilibria, by which we mean an open-loop Nash equilibrium $u^{* *}(\cdot) \equiv\left(u_{1}^{* *}(\cdot), u_{2}^{* *}(\cdot)\right)$ admits a form (3.9) for some $\left(\mathbf{\Theta}^{* *}, v^{* *}(\cdot)\right) \in$ $\mathscr{S}[A, \bar{A}, C, \bar{C} ; B, \bar{B}, D, \bar{D}] \times L_{\mathbb{F}}^{2}\left(\mathbb{R}^{m}\right)$. We state the result here and put a detailed proof in [29]. 
Theorem 3.4. Let (H2) hold. Then an open-loop Nash equilibria $u^{* *}(\cdot) \equiv\left(u_{1}^{* *}(\cdot), u_{2}^{* *}(\cdot)\right) \in \mathscr{U}_{a d}(x)$ of Problem (MF-SDG) admits the following closed-loop representation

$$
u_{i}^{* *}(\cdot)=\Theta_{i}^{* *}\{X(\cdot)-\mathbb{E}[X(\cdot)]\}+\bar{\Theta}_{i}^{* *} \mathbb{E}[X(\cdot)]+v_{i}^{* *}(\cdot), \quad i=1,2,
$$

with $\Theta^{* *} \equiv\left(\begin{array}{c}\Theta_{1}^{* *} \\ \Theta_{2}^{* *}\end{array}\right) \equiv\left(\begin{array}{cc}\Theta_{1}^{* *} & \bar{\Theta}_{1}^{* *} \\ \Theta_{2}^{* *} & \bar{\Theta}_{2}^{* *}\end{array}\right) \in \mathscr{S}[A, \bar{A}, C, \bar{C} ; B, \bar{B}, D, \bar{D}]$ and $v^{* *}(\cdot) \equiv\left(\begin{array}{c}v_{1}^{* *}(\cdot) \\ v_{2}^{* *}(\cdot)\end{array}\right) \in L_{\mathbb{F}}^{2}\left(\mathbb{R}^{m}\right)$ if and only if the following hold:

(i) The convexity condition (3.19) holds for $i=1,2$.

(ii) The solution pair $(\mathbf{P}, \widehat{\mathbf{P}}) \triangleq\left(\begin{array}{ll}P_{1} & \widehat{P}_{1} \\ P_{2} & \widehat{P}_{2}\end{array}\right) \in \mathbb{R}^{2 n \times 2 n}$ to the system of coupled AREs:

$$
\left\{\begin{array}{l}
\mathbf{P} A+\mathbf{A}^{\top} \mathbf{P}+\mathbf{C}^{\top} \mathbf{P} C+\mathbf{Q} \mathbf{I}_{n}-\left(\mathbf{P} B+\mathbf{C}^{\top} \mathbf{P} D+\mathbf{S}^{\top} \mathbf{I}_{m}\right) \mathbf{\Sigma}^{-1} \mathbf{J}^{\top}\left(\mathbf{B}^{\top} \mathbf{P}+\mathbf{D}^{\top} \mathbf{P} C+\mathbf{S} \mathbf{I}_{n}\right)=0 \\
\widehat{\mathbf{P}} \widehat{A}+\widehat{\mathbf{A}}^{\top} \widehat{\mathbf{P}}+\widehat{\mathbf{C}}^{\top} \mathbf{P} \widehat{C}+\widehat{\mathbf{Q}} \mathbf{I}_{n}-\left(\widehat{\mathbf{P}} \widehat{B}+\widehat{\mathbf{C}}^{\top} \mathbf{P} \widehat{D}+\widehat{\mathbf{S}}^{\top} \mathbf{I}_{m}\right) \overline{\boldsymbol{\Sigma}}^{-1} \mathbf{J}^{\top}\left(\widehat{\mathbf{B}}^{\top} \widehat{\mathbf{P}}+\widehat{\mathbf{D}}^{\top} \mathbf{P} \widehat{C}+\widehat{\mathbf{S}} \mathbf{I}_{n}\right)=0
\end{array}\right.
$$

where $\widehat{\mathbf{A}}=\mathbf{A}+\overline{\mathbf{A}}$ and $\widehat{\mathbf{C}}, \widehat{\mathbf{Q}}, \widehat{\mathbf{S}}$ are defined similarly as (2.15) and

$$
\boldsymbol{\Sigma} \triangleq \mathbf{J}^{\top}\left(\mathbf{R} \mathbf{I}_{m}+\mathbf{D}^{\top} \mathbf{P} D\right), \quad \overline{\boldsymbol{\Sigma}} \triangleq \mathbf{J}^{\top}\left(\widehat{\mathbf{R}} \mathbf{I}_{m}+\widehat{\mathbf{D}}^{\top} \mathbf{P} \widehat{D}\right) \in \mathbb{R}^{m \times m}
$$

are both invertible such that $\boldsymbol{\Theta}^{* *} \equiv\left(\Theta^{* *}, \bar{\Theta}^{* *}\right) \equiv\left(\begin{array}{cc}\Theta_{1}^{* *} & \bar{\Theta}_{1}^{* *} \\ \Theta_{2}^{* *} & \bar{\Theta}_{2}^{* *}\end{array}\right) \in \mathbb{R}^{m \times 2 n}$ defined by

$$
\Theta^{* *} \triangleq-\boldsymbol{\Sigma}^{-1} \mathbf{J}^{\top}\left(\mathbf{B}^{\top} \mathbf{P}+\mathbf{D}^{\top} \mathbf{P} C+\mathbf{S I}_{n}\right), \quad \bar{\Theta}^{* *} \triangleq-\overline{\boldsymbol{\Sigma}}^{-1} \mathbf{J}^{\top}\left(\widehat{\mathbf{B}}^{\top} \widehat{\mathbf{P}}+\widehat{\mathbf{D}}^{\top} \mathbf{P} \widehat{C}+\widehat{\mathbf{S}} \mathbf{I}_{n}\right),
$$

stabilizes the system $[A, \bar{A}, C, \bar{C} ; B, \bar{B}, D, \bar{D}]$. Also,

$$
\begin{aligned}
v^{* *}(\cdot)= & -\boldsymbol{\Sigma}^{-1} \mathbf{J}^{\top}\left\{\mathbf{B}^{\top}(\eta-\mathbb{E}[\eta])+\mathbf{D}^{\top}(\zeta-\mathbb{E}[\zeta])+\mathbf{D}^{\top} \mathbf{P}(\sigma-\mathbb{E}[\sigma])+\rho-\mathbb{E}[\rho]\right\} \\
& -\overline{\boldsymbol{\Sigma}}^{-1} \mathbf{J}^{\top}\left\{\widehat{\mathbf{B}}^{\top} \bar{\eta}+\widehat{\mathbf{D}}^{\top} \mathbb{E}[\zeta]+\widehat{\mathbf{D}}^{\top} \mathbf{P} \mathbb{E}[\sigma]+\mathbb{E}[\rho]\right\} \in L_{\mathbb{F}}^{2}\left(\mathbb{R}^{m}\right),
\end{aligned}
$$

where $\left(\eta(\cdot) \triangleq\left(\begin{array}{l}\eta_{1}(\cdot) \\ \eta_{2}(\cdot)\end{array}\right), \zeta(\cdot) \triangleq\left(\begin{array}{l}\zeta_{1}(\cdot) \\ \zeta_{2}(\cdot)\end{array}\right)\right) \in \mathscr{X}[0, \infty)^{2} \times L_{\mathbb{F}}^{2}\left(\mathbb{R}^{2 n}\right)$ is an adapted solution to BSDE:

$$
\begin{aligned}
-\mathrm{d} \eta(t)=\{ & \mathbf{A}^{\top} \eta(t)-\left(\mathbf{P} B+\mathbf{C}^{\top} \mathbf{P} D+\mathbf{S}^{\top} \mathbf{I}_{m}\right) \boldsymbol{\Sigma}^{-1} \mathbf{J}^{\top}\left\{\mathbf{B}^{\top} \eta(t)+\mathbf{D}^{\top}[\zeta(t)+\mathbf{P} \sigma(t)]+\rho(t)\right\} \\
& \left.+\mathbf{C}^{\top}[\zeta(t)+\mathbf{P} \sigma(t)]+\mathbf{P} b(t)+q(t)\right\} \mathrm{d} t-\zeta(t) \mathrm{d} W(t), \quad t \geqslant 0,
\end{aligned}
$$

and $\bar{\eta}(\cdot) \triangleq\left(\begin{array}{l}\bar{\eta}_{1}(\cdot) \\ \bar{\eta}_{2}(\cdot)\end{array}\right) \in L^{2}\left(\mathbb{R}^{2 n}\right)$ is a solution to ODE:

$$
\begin{aligned}
\dot{\bar{\eta}}+ & \widehat{\mathbf{A}}^{\top} \bar{\eta}+\widehat{\mathbf{C}}^{\top} \mathbb{E}[\zeta+\mathbf{P} \sigma]+\widehat{\mathbf{P}} \mathbb{E}[b]+\mathbb{E}[q] \\
& -\left(\widehat{\mathbf{P}} \widehat{B}+\widehat{\mathbf{C}}^{\top} \mathbf{P} \widehat{D}+\widehat{\mathbf{S}}^{\top} \mathbf{I}_{m}\right) \overline{\boldsymbol{\Sigma}}^{-1}\left\{\widehat{\mathbf{B}}^{\top} \bar{\eta}+\widehat{\mathbf{D}}^{\top} \mathbb{E}[\zeta+\mathbf{P} \sigma]+\mathbb{E}[\rho]\right\}=0, \quad t \geqslant 0
\end{aligned}
$$

In such a case, the open-loop Nash equilibrium $u^{* *}(\cdot)$ admits a closed-loop representation (3.25) with $\left(\boldsymbol{\Theta}^{* *}, v^{* *}(\cdot)\right)$ given by the above. 
Note that by the definition of $\Theta^{* *}$ and $\bar{\Theta}^{* *}$ in $(3.27)$, we have

$$
\mathbf{\Sigma} \Theta^{* *}+\mathbf{J}^{\top}\left(\mathbf{B}^{\top} \mathbf{P}+\mathbf{D}^{\top} \mathbf{P} C+\mathbf{S I}_{n}\right)=0, \quad \bar{\Sigma} \bar{\Theta}^{* *}+\mathbf{J}^{\top}\left(\widehat{\mathbf{B}}^{\top} \widehat{\mathbf{P}}+\widehat{\mathbf{D}}^{\top} \mathbf{P} \widehat{C}+\widehat{\mathbf{S}} \mathbf{I}_{n}\right)=0
$$

and we can rewrite $(3.26)$ as

$$
\left\{\begin{array}{l}
\mathbf{P} A+\mathbf{A}^{\top} \mathbf{P}+\mathbf{C}^{\top} \mathbf{P} C+\mathbf{Q} \mathbf{I}_{n}+\left(\mathbf{P} B+\mathbf{C}^{\top} \mathbf{P} D+\mathbf{S}^{\top} \mathbf{I}_{m}\right) \Theta^{* *}=0 \\
\widehat{\mathbf{P}} \widehat{A}+\widehat{\mathbf{A}}^{\top} \widehat{\mathbf{P}}+\widehat{\mathbf{C}}^{\top} \mathbf{P} \widehat{C}+\widehat{\mathbf{Q}} \mathbf{I}_{n}+\left(\widehat{\mathbf{P}} \widehat{B}+\widehat{\mathbf{C}}^{\top} \mathbf{P} \widehat{D}+\widehat{\mathbf{S}}^{\top} \mathbf{I}_{m}\right) \bar{\Theta}^{* *}=0
\end{array}\right.
$$

We may further write (3.31) and (3.32) in the component forms:

$$
\begin{gathered}
\left\{\begin{array}{l}
P_{i} A+A^{\top} P_{i}+C^{\top} P_{i} C+Q_{i}+\left(P_{i} B+C^{\top} P_{i} D+S_{i}^{\top}\right) \Theta^{* *}=0, \\
\widehat{P}_{i} \widehat{A}+\widehat{A}^{\top} \widehat{P}_{i}+\widehat{C}^{\top} P_{i} \widehat{C}+\widehat{Q}_{i}+\left(\widehat{P}_{i} \widehat{B}+\widehat{C}^{\top} P_{i} \widehat{D}+\widehat{S}_{i}^{\top}\right) \bar{\Theta}^{* *}=0,
\end{array}=1,2,\right. \\
\left\{\begin{array}{ll}
R_{111}+D_{1}^{\top} P_{1} D_{1} & R_{112}+D_{1}^{\top} P_{1} D_{2} \\
R_{221}+D_{2}^{\top} P_{2} D_{1} & R_{222}+D_{2}^{\top} P_{2} D_{2}
\end{array}\right) \Theta^{* *}+\left(\begin{array}{l}
B_{1}^{\top} P_{1}+D_{1}^{\top} P_{1} C+S_{11} \\
B_{2}^{\top} P_{2}+D_{2}^{\top} P_{2} C+S_{22}
\end{array}\right)=0, \\
\left(\begin{array}{ll}
\widehat{R}_{111}+\widehat{D}_{1}^{\top} P_{1} \widehat{D}_{1} & \widehat{R}_{112}+\widehat{D}_{1}^{\top} P_{1} \widehat{D}_{2} \\
\widehat{R}_{221}+\widehat{D}_{2}^{\top} P_{2} \widehat{D}_{1} & \widehat{R}_{222}+\widehat{D}_{2}^{\top} P_{2} \widehat{D}_{2}
\end{array}\right) \bar{\Theta}^{* *}+\left(\begin{array}{l}
\widehat{B}_{1}^{\top} \widehat{P}_{1}+\widehat{D}_{1}^{\top} P_{1} \widehat{C}+\widehat{S}_{11} \\
\widehat{B}_{2}^{\top} \widehat{P}_{2}+\widehat{D}_{2}^{\top} P_{2} \widehat{C}+\widehat{S}_{22}
\end{array}\right)=0 .
\end{gathered}
$$

In the above, the coefficient matrices of the equations for $\Theta^{* *}$ and $\bar{\Theta}^{* *}$ are not symmetric in general (even if $P_{1}, P_{2}, \widehat{P}_{1}, \widehat{P}_{2}$ are all symmetric). Hence, the equations for $P_{i}, \widehat{P}_{i}, i=1,2$ and for $P_{i}^{\top}, \widehat{P}_{i}^{\top}, i=1,2$ are different. Consequently, we do not expect $P_{i}, \widehat{P}_{i}, i=1,2$ to be symmetric in general.

\subsection{Closed-loop Nash equalibria and symmetric algebraic Riccati equations}

We now look at closed-loop Nash equilibria for Problem (MF-SDG). First, we present the following result, which is a consequence of Theorem 3.3 and its proof is similar to that of Proposition 5.1 in [47] (see also [29]).

Proposition 3.5. Let (H2) hold. If $\left(\Theta^{*}, v^{*}(\cdot)\right)$ is a closed-loop Nash equilibrium of Problem (MF-SDG), then $\left(\mathbf{\Theta}^{*}, 0\right)$ is a closed-loop Nash equilibrium of Problem (MF-SDG) ${ }^{0}$.

Now, we give a necessary condition for closed-loop Nash equilibria of Problem (MF-SDG).

Proposition 3.6. Let (H2) hold, and let $\left(\mathbf{\Theta}^{*}, v^{*}(\cdot)\right)$ be a closed-loop Nash equilibrium of Problem (MF-SDG). Then for $i=1,2$, the following system of coupled AREs admits a solution pair $\left(P_{i}, \widehat{P}_{i}\right) \in \mathbb{S}^{n} \times \mathbb{S}^{n}$ :

$$
\left\{\begin{array}{c}
P_{i} A+A^{\top} P_{i}+C^{\top} P_{i} C+Q_{i}+\left(\Theta^{*}\right)^{\top}\left(R_{i}+D^{\top} P_{i} D\right) \Theta^{*} \\
\quad+\left(P_{i} B+C^{\top} P_{i} D+S_{i}^{\top}\right) \Theta^{*}+\left(\Theta^{*}\right)^{\top}\left(B^{\top} P_{i}+D^{\top} P_{i} C+S_{i}\right)=0 \\
\widehat{P}_{i} \widehat{A}+\widehat{A}^{\top} \widehat{P}_{i}+\widehat{C}^{\top} P_{i} \widehat{C}+\widehat{Q}_{i}+\left(\bar{\Theta}^{*}\right)^{\top}\left(\widehat{R}_{i}+\widehat{D}^{\top} P_{i} \widehat{D}\right) \bar{\Theta}^{*} \\
\quad+\left(\widehat{P}_{i} \widehat{B}+\widehat{C}^{\top} P_{i} \widehat{D}+\widehat{S}_{i}^{\top}\right) \bar{\Theta}^{*}+\left(\bar{\Theta}^{*}\right)^{\top}\left(\widehat{B}^{\top} \widehat{P}_{i}+\widehat{D}^{\top} P_{i} \widehat{C}+\widehat{S}_{i}\right)=0
\end{array}\right.
$$

and the following conditions are satisfied:

$$
\left\{\begin{array}{l}
B_{i}^{\top} P_{i}+D_{i}^{\top} P_{i} C+S_{i i}+\Sigma_{i}\left(R_{i i}+D_{i}^{\top} P_{i} D\right) \Theta^{*}=0 \\
\widehat{B}_{i}^{\top} \widehat{P}_{i}+\widehat{D}_{i}^{\top} P_{i} \widehat{C}+\widehat{S}_{i i}+\left(\widehat{R}_{i i}+\widehat{D}_{i}^{\top} P_{i} \widehat{D}\right) \bar{\Theta}^{*}=0
\end{array}\right.
$$


with

$$
\Sigma_{i} \triangleq R_{i i i}+D_{i}^{\top} P_{i} D_{i} \geqslant 0, \quad \bar{\Sigma}_{i} \triangleq \widehat{R}_{i i i}+\widehat{D}_{i}^{\top} P_{i} \widehat{D}_{i} \geqslant 0
$$

Proof. Suppose that $\left(\boldsymbol{\Theta}^{*}, v^{*}(\cdot)\right)$ is a closed-loop Nash equilibrium of Problem (MF-SDG). Then by Proposition $3.5,\left(\Theta^{*}, 0\right)$ is a closed-loop Nash equilibrium of Problem $(\mathrm{MF}-\mathrm{SDG})^{0}$. Denote

$$
\left\{\begin{array}{l}
\mathcal{A}_{1} \triangleq A+B_{2} \Theta_{2}^{*}, \quad \overline{\mathcal{A}}_{1} \triangleq \bar{A}+\bar{B}_{2} \bar{\Theta}_{2}^{*}+B_{2}\left(\bar{\Theta}_{2}^{*}-\Theta_{2}^{*}\right), \quad \mathcal{C}_{1} \triangleq C+D_{2} \Theta_{2}^{*}, \\
\overline{\mathcal{C}}_{1} \triangleq \bar{C}+\bar{D}_{2} \bar{\Theta}_{2}^{*}+D_{2}\left(\bar{\Theta}_{2}^{*}-\Theta_{2}^{*}\right), \quad \mathcal{Q}_{1} \triangleq Q_{1}+S_{12}^{\top} \Theta_{2}^{*}+\left(\Theta_{2}^{*}\right)^{\top} S_{12}+\left(\Theta_{2}^{*}\right)^{\top} R_{122} \Theta_{2}^{*}, \\
\overline{\mathcal{Q}}_{1} \triangleq \bar{Q}_{1}+\widehat{S}_{12}^{\top} \bar{\Theta}_{2}^{*}+\left(\bar{\Theta}_{2}^{*}\right)^{\top} \widehat{S}_{12}+\left(\bar{\Theta}_{2}^{*}\right)^{\top} \widehat{R}_{122} \bar{\Theta}_{2}^{*}-S_{12}^{\top} \Theta_{2}^{*}-\left(\Theta_{2}^{*}\right)^{\top} S_{12}-\left(\Theta_{2}^{*}\right)^{\top} R_{122} \Theta_{2}^{*}, \\
\mathcal{S}_{11} \triangleq S_{11}+R_{112} \Theta_{2}^{*}, \quad \overline{\mathcal{S}}_{11} \triangleq \bar{S}_{11}+\bar{R}_{112} \bar{\Theta}_{2}^{*}+R_{112}\left(\bar{\Theta}_{2}^{*}-\Theta_{2}^{*}\right) .
\end{array}\right.
$$

Then, for any $u_{1}(\cdot) \in L_{\mathbb{F}}^{2}\left(\mathbb{R}^{m_{1}}\right)$, let us consider the state equation:

$$
\left\{\begin{array}{l}
\mathrm{d} X_{1}^{0}(t)=\left\{\mathcal{A}_{1} X_{1}^{0}(t)+\overline{\mathcal{A}}_{1} \mathbb{E}\left[X_{1}^{0}(t)\right]+B_{1} u_{1}(t)+\bar{B}_{1} \mathbb{E}\left[u_{1}(t)\right]\right\} \mathrm{d} t \\
\quad+\left\{\mathcal{C}_{1} X_{1}^{0}(t)+\overline{\mathcal{C}}_{1} \mathbb{E}\left[X_{1}^{0}(t)\right]+D_{1} u_{1}(t)+\bar{D}_{1} \mathbb{E}\left[u_{1}(t)\right]\right\} \mathrm{d} W(t), \quad t \geqslant 0, \\
X_{1}^{0}(0)=0,
\end{array}\right.
$$

and cost functional:

$$
\begin{aligned}
& \bar{J}_{1}^{0}\left(x ; u_{1}(\cdot)\right) \equiv J_{1}^{0}\left(x ; u_{1}(\cdot) ; \Theta_{2}^{*}\left\{X_{1}^{0}(\cdot)-\mathbb{E}\left[X_{1}^{0}(\cdot)\right]\right\}+\bar{\Theta}_{2}^{*} \mathbb{E}\left[X_{1}^{0}(\cdot)\right]\right) \\
& =\mathbb{E} \int_{0}^{\infty}\left[\left\langle\left(\begin{array}{cc}
\mathcal{Q}_{1} & \mathcal{S}_{11}^{\top} \\
\mathcal{S}_{11} & R_{111}
\end{array}\right)\left(\begin{array}{c}
X_{1}^{0} \\
u_{1}
\end{array}\right),\left(\begin{array}{c}
X_{1}^{0} \\
u_{1}
\end{array}\right)\right\rangle+\left\langle\left(\begin{array}{cc}
\overline{\mathcal{Q}}_{1} & \overline{\mathcal{S}}_{11}^{\top} \\
\overline{\mathcal{S}}_{11} & \bar{R}_{111}
\end{array}\right)\left(\begin{array}{c}
\mathbb{E}\left[X_{1}^{0}\right] \\
\mathbb{E}\left[u_{1}\right]
\end{array}\right),\left(\begin{array}{c}
\mathbb{E}\left[X_{1}^{0}\right] \\
\mathbb{E}\left[u_{1}\right]
\end{array}\right)\right\rangle\right] \mathrm{d} t .
\end{aligned}
$$

It is easy to see that $\left(\boldsymbol{\Theta}_{1}^{*}, 0\right)$ is a closed-loop optimal strategy for the above mean-field LQ stochastic optimal control problem. Thanks to Theorem 2.9, the following system of coupled AREs:

$$
\left\{\begin{array}{l}
P_{1} \mathcal{A}_{1}+\mathcal{A}_{1}^{\top} P_{1}+\mathcal{C}_{1}^{\top} P_{1} \mathcal{C}_{1}+\mathcal{Q}_{1}-\left(P_{1} B_{1}+\mathcal{C}_{1}^{\top} P_{1} D_{1}+\mathcal{S}_{11}^{\top}\right) \Sigma_{1}^{\dagger}\left(B_{1}^{\top} P_{1}+D_{1}^{\top} P_{1} \mathcal{C}_{1}+\mathcal{S}_{11}\right)=0 \\
\widehat{P}_{1} \widehat{\mathcal{A}}_{1}+\widehat{\mathcal{A}}_{1}^{\top} \widehat{P}_{1}+\widehat{\mathcal{C}}_{1}^{\top} P_{1} \widehat{\mathcal{C}}_{1}+\widehat{\mathcal{Q}}_{1}-\left(\widehat{P}_{1} \widehat{B}_{1}+\widehat{\mathcal{C}}_{1}^{\top} P_{1} \widehat{D}_{1}+\widehat{\mathcal{S}}_{11}^{\top}\right) \Sigma_{1}^{\dagger}\left(\widehat{B}_{1}^{\top} \widehat{P}_{1}+\widehat{D}_{1}^{\top} P_{1} \widehat{\mathcal{C}}_{1}+\widehat{\mathcal{S}}_{11}\right)=0
\end{array}\right.
$$

admit a static stabilizing solution pair $\left(P_{1}, \widehat{P}_{1}\right) \in \mathbb{S}^{n} \times \mathbb{S}^{n}$, satisfying

$$
\begin{cases}B_{1}^{\top} P_{1}+D_{1}^{\top} P_{1} \mathcal{C}_{1}+\mathcal{S}_{11}+\Sigma_{1} \Theta_{1}^{*}=0, & \Sigma_{1} \equiv R_{111}+D_{1}^{\top} P_{1} D_{1} \geqslant 0 \\ \widehat{B}_{1}^{\top} \widehat{P}_{1}+\widehat{D}_{1}^{\top} P_{1} \widehat{\mathcal{C}}_{1}+\widehat{\mathcal{S}}_{11}+\bar{\Sigma}_{1} \bar{\Theta}_{1}^{*}=0, & \bar{\Sigma}_{1} \equiv \widehat{R}_{111}+\widehat{D}_{1}^{\top} P_{1} \widehat{D}_{1} \geqslant 0\end{cases}
$$

where $\widehat{\mathcal{A}}_{1}=\mathcal{A}+\overline{\mathcal{A}}_{1}, \widehat{\mathcal{C}}_{1}=\mathcal{C}_{1}+\overline{\mathcal{C}}_{1}, \widehat{\mathcal{Q}}_{1}=\mathcal{Q}_{1}+\overline{\mathcal{Q}}_{1}$, and $\widehat{\mathcal{S}}_{11}=\mathcal{S}_{11}+\overline{\mathcal{S}}_{11}$. Similarly, for any $u_{2}(\cdot) \in L_{\mathbb{F}}^{2}\left(\mathbb{R}^{m_{2}}\right)$, we can consider the state equation:

$$
\left\{\begin{aligned}
& \mathrm{d} X_{2}^{0}(t)=\{\left.\mathcal{A}_{2} X_{2}^{0}(t)+\overline{\mathcal{A}}_{2} \mathbb{E}\left[X_{2}^{0}(t)\right]+B_{2} u_{2}(t)+\bar{B}_{2} \mathbb{E}\left[u_{2}(t)\right]\right\} \mathrm{d} t \\
&+\left\{\mathcal{C}_{2} X_{2}^{0}(t)+\overline{\mathcal{C}}_{2} \mathbb{E}\left[X_{2}^{0}(t)\right]+D_{2} u_{2}(t)+\bar{D}_{2} \mathbb{E}\left[u_{2}(t)\right]\right\} \mathrm{d} W(t), \quad t \geqslant 0, \\
& X_{2}^{0}(0)=0,
\end{aligned}\right.
$$


and cost functional:

$$
\begin{aligned}
& \bar{J}_{2}^{0}\left(x ; u_{2}(\cdot)\right) \equiv J_{2}^{0}\left(x ; \Theta_{1}^{*}\left(X_{2}^{0}(\cdot)-\mathbb{E}\left[X_{2}^{0}(\cdot)\right]\right)+\bar{\Theta}_{1}^{*} \mathbb{E}\left[X_{2}^{0}(\cdot)\right] ; u_{2}(\cdot)\right) \\
& =\mathbb{E} \int_{0}^{\infty}\left[\left\langle\left(\begin{array}{cc}
\mathcal{Q}_{2} & \mathcal{S}_{22}^{\top} \\
\mathcal{S}_{22} & R_{222}
\end{array}\right)\left(\begin{array}{c}
X_{2}^{0} \\
u_{2}
\end{array}\right),\left(\begin{array}{c}
X_{2}^{0} \\
u_{2}
\end{array}\right)\right\rangle+\left\langle\left(\begin{array}{cc}
\overline{\mathcal{Q}}_{2} & \overline{\mathcal{S}}_{22}^{\top} \\
\overline{\mathcal{S}}_{22} & \bar{R}_{22}
\end{array}\right)\left(\begin{array}{c}
\mathbb{E}\left[X_{2}^{0}\right] \\
\mathbb{E}\left[u_{2}\right]
\end{array}\right),\left(\begin{array}{c}
\mathbb{E}\left[X_{2}^{0}\right] \\
\mathbb{E}\left[u_{2}\right]
\end{array}\right)\right\rangle\right] \mathrm{d} t
\end{aligned}
$$

where $\mathcal{A}_{2}, \overline{\mathcal{A}}_{2}, \mathcal{C}_{2}, \overline{\mathcal{C}}_{2}$ and $\mathcal{Q}_{2}, \overline{\mathcal{Q}}_{2}, \mathcal{S}_{22}, \overline{\mathcal{S}}_{22}$ are defined similar to (3.38). In the same spirit as above, we can see that $\left(\Theta_{2}^{*}, 0\right) \equiv\left(\Theta_{2}^{*}, \bar{\Theta}_{2}^{*}, 0\right)$ is a closed-loop optimal strategy for the above mean-field LQ stochastic optimal control problem. Making use of Theorem 2.9 again, the following system of coupled AREs

$$
\left\{\begin{array}{l}
P_{2} \mathcal{A}_{2}+\mathcal{A}_{2}^{\top} P_{2}+\mathcal{C}_{2}^{\top} P_{2} \mathcal{C}_{2}+\mathcal{Q}_{2}-\left(P_{2} B_{2}+\mathcal{C}_{2}^{\top} P_{2} D_{2}+\mathcal{S}_{22}^{\top}\right) \Sigma_{2}^{\dagger}\left(B_{2}^{\top} P_{2}+D_{2}^{\top} P_{2} \mathcal{C}_{2}+\mathcal{S}_{22}\right)=0 \\
\widehat{P}_{2} \widehat{\mathcal{A}}_{2}+\widehat{\mathcal{A}}_{2}^{\top} \widehat{P}_{2}+\widehat{\mathcal{C}}_{2}^{\top} P_{2} \widehat{\mathcal{C}}_{2}+\widehat{\mathcal{Q}}_{2}-\left(\widehat{P}_{2} \widehat{B}_{2}+\widehat{\mathcal{C}}_{2}^{\top} P_{2} \widehat{D}_{2}+\widehat{\mathcal{S}}_{22}^{\top}\right) \bar{\Sigma}_{2}^{\dagger}\left(\widehat{B}_{2}^{\top} \widehat{P}_{2}+\widehat{D}_{2}^{\top} P_{2} \widehat{\mathcal{C}}_{2}+\widehat{\mathcal{S}}_{22}\right)=0
\end{array}\right.
$$

admit a static stabilizing solution pair $\left(P_{2}, \widehat{P}_{2}\right) \in \mathbb{S}^{n} \times \mathbb{S}^{n}$, satisfying

$$
\begin{cases}0=B_{2}^{\top} P_{2}+D_{2}^{\top} P_{2} \mathcal{C}_{2}+\mathcal{S}_{22}+\Sigma_{2} \Theta_{2}^{*}, & \Sigma_{2} \equiv R_{222}+D_{2}^{\top} P_{2} D_{2} \geqslant 0 \\ 0=\widehat{B}_{2}^{\top} \widehat{P}_{2}+\widehat{D}_{2}^{\top} P_{2} \widehat{\mathcal{C}}_{2}+\widehat{\mathcal{S}}_{22}+\bar{\Sigma}_{2} \bar{\Theta}_{2}^{*}, & \bar{\Sigma}_{2} \equiv \widehat{R}_{222}+\widehat{D}_{2}^{\top} P_{2} \widehat{D}_{2} \geqslant 0\end{cases}
$$

where $\widehat{\mathcal{A}}_{2}=\mathcal{A}_{2}+\overline{\mathcal{A}}_{2}, \widehat{\mathcal{C}}_{2}=\mathcal{C}_{2}+\overline{\mathcal{C}}_{2}, \widehat{\mathcal{Q}}_{2}=\mathcal{Q}_{2}+\overline{\mathcal{Q}}_{2}$, and $\widehat{\mathcal{S}}_{22}=\mathcal{S}_{22}+\overline{\mathcal{S}}_{22}$. By (3.40) and (3.42) and putting $\Theta^{*} \equiv\left(\Theta^{*}, \bar{\Theta}^{*}\right)=\left(\begin{array}{cc}\Theta_{1}^{*} & \bar{\Theta}_{1}^{*} \\ \Theta_{2}^{*} & \bar{\Theta}_{2}^{*}\end{array}\right) \in \mathbb{R}^{m \times 2 n}$, we get $(3.37)$ and

$$
\left\{\begin{array}{l}
0=B_{1}^{\top} P_{1}+D_{1}^{\top} P_{1} \mathcal{C}_{1}+\mathcal{S}_{11}+\Sigma_{1} \Theta_{1}^{*}=B_{1}^{\top} P_{1}+D_{1}^{\top} P_{1} C+S_{11}+\left(R_{11}+D_{1}^{\top} P_{1} D\right) \Theta^{*} \\
0=\widehat{B}_{1}^{\top} \widehat{P}_{1}+\widehat{D}_{1}^{\top} P_{1} \widehat{\mathcal{C}}_{1}+\widehat{\mathcal{S}}_{11}+\bar{\Sigma}_{1} \bar{\Theta}_{1}^{*}=\widehat{B}_{1}^{\top} \widehat{P}_{1}+\widehat{D}_{1}^{\top} P_{1} \widehat{C}+\widehat{S}_{11}+\left(\widehat{R}_{11}+\widehat{D}_{1}^{\top} P_{1} \widehat{D}\right) \bar{\Theta}^{*}
\end{array}\right.
$$

Similarly,

$$
\left\{\begin{array}{l}
0=B_{2}^{\top} P_{2}+D_{2}^{\top} P_{2} \mathcal{C}_{2}+\mathcal{S}_{22}+\Sigma_{2} \Theta_{2}^{*}=B_{2}^{\top} P_{2}+D_{2}^{\top} P_{2} C+S_{22}+\left(R_{22}+D_{2}^{\top} P_{2} D\right) \Theta^{*} \\
0=\widehat{B}_{2}^{\top} \widehat{P}_{2}+\widehat{D}_{2}^{\top} P_{2} \widehat{\mathcal{C}}_{2}+\widehat{\mathcal{S}}_{22}+\bar{\Sigma}_{2} \bar{\Theta}_{2}^{*}=\widehat{B}_{2}^{\top} \widehat{P}_{2}+\widehat{D}_{2}^{\top} P_{2} \widehat{C}+\widehat{S}_{22}+\left(\widehat{R}_{22}+\widehat{D}_{2}^{\top} P_{2} \widehat{D}\right) \bar{\Theta}^{*}
\end{array}\right.
$$

which implies (3.36). By (3.36), (3.39) and (3.41), we have (by a straightforward calculation)

$$
\left\{\begin{aligned}
0= & P_{1} \mathcal{A}_{1}+\mathcal{A}_{1}^{\top} P_{1}+\mathcal{C}_{1}^{\top} P_{1} \mathcal{C}_{1}+\mathcal{Q}_{1}-\left(P_{1} B_{1}+\mathcal{C}_{1}^{\top} P_{1} D_{1}+\mathcal{S}_{11}^{\top}\right) \Sigma_{1}^{\dagger}\left(B_{1}^{\top} P_{1}+D_{1}^{\top} P_{1} \mathcal{C}_{1}+\mathcal{S}_{11}\right) \\
= & P_{1} A+A^{\top} P_{1}+C^{\top} P_{1} C+Q_{1}+\left(\Theta^{*}\right)^{\top}\left(R_{1}+D^{\top} P_{1} D\right) \Theta^{*} \\
& +\left(P_{1} B+C^{\top} P_{1} D+S_{1}^{\top}\right) \Theta^{*}+\left(\Theta^{*}\right)^{\top}\left(B^{\top} P_{1}+D^{\top} P_{1} C+S_{1}\right), \\
0 & \widehat{P}_{1} \widehat{\mathcal{A}}_{1}+\widehat{\mathcal{A}}_{1}^{\top} \widehat{P}_{1}+\widehat{\mathcal{C}}_{1}^{\top} P_{1} \widehat{\mathcal{C}}_{1}+\widehat{\mathcal{Q}}_{1}-\left(\widehat{P}_{1} \widehat{B}_{1}+\widehat{\mathcal{C}}_{1}^{\top} P_{1} \widehat{D}_{1}+\widehat{\mathcal{S}}_{11}^{\top}\right) \bar{\Sigma}_{1}^{\dagger}\left(\widehat{B}_{1}^{\top} \widehat{P}_{1}+\widehat{D}_{1}^{\top} P_{1} \widehat{\mathcal{C}}_{1}+\widehat{\mathcal{S}}_{11}\right) \\
= & \widehat{P}_{1} \widehat{A}+\widehat{A}^{\top} \widehat{P}_{1}+\widehat{C}^{\top} P_{1} \widehat{C}+\widehat{Q}_{1}+\left(\bar{\Theta}^{*}\right)^{\top}\left(\widehat{R}_{1}+\widehat{D}^{\top} P_{1} \widehat{D}\right) \bar{\Theta}^{*} \\
& +\left(\widehat{P}_{1} \widehat{B}+\widehat{C}^{\top} P_{1} \widehat{D}+\widehat{S}_{1}^{\top}\right) \bar{\Theta}^{*}+\left(\bar{\Theta}^{*}\right)^{\top}\left(\widehat{B}^{\top} \widehat{P}_{1}+\widehat{D}^{\top} P_{1} \widehat{C}+\widehat{S}_{1}\right) .
\end{aligned}\right.
$$


In the same way, we have

$$
\left\{\begin{aligned}
0= & P_{2} \mathcal{A}_{2}+\mathcal{A}_{2}^{\top} P_{2}+\mathcal{C}_{2}^{\top} P_{2} \mathcal{C}_{2}+\mathcal{Q}_{2}-\left(P_{2} B_{2}+\mathcal{C}_{2}^{\top} P_{2} D_{2}+\mathcal{S}_{22}^{\top}\right) \Sigma_{2}^{\dagger}\left(B_{2}^{\top} P_{2}+D_{2}^{\top} P_{2} \mathcal{C}_{2}+\mathcal{S}_{22}\right) \\
= & P_{2} A+A^{\top} P_{2}+C^{\top} P_{2} C+Q_{2}+\left(\Theta^{*}\right)^{\top}\left(R_{2}+D^{\top} P_{2} D\right) \Theta^{*} \\
& +\left(P_{2} B+C^{\top} P_{2} D+S_{2}^{\top}\right) \Theta^{*}+\left(\Theta^{*}\right)^{\top}\left(B^{\top} P_{2}+D^{\top} P_{2} C+S_{2}\right), \\
0 & \widehat{P}_{2} \widehat{\mathcal{A}}_{2}+\widehat{\mathcal{A}}_{2}^{\top} \widehat{P}_{2}+\widehat{\mathcal{C}}_{2}^{\top} P_{2} \widehat{\mathcal{C}}_{2}+\widehat{\mathcal{Q}}_{2}-\left(\widehat{P}_{2} \widehat{B}_{2}+\widehat{\mathcal{C}}_{2}^{\top} P_{2} \widehat{D}_{2}+\widehat{\mathcal{S}}_{22}^{\top}\right) \bar{\Sigma}_{2}^{\dagger}\left(\widehat{B}_{2}^{\top} \widehat{P}_{2}+\widehat{D}_{2}^{\top} P_{2} \widehat{\mathcal{C}}_{2}+\widehat{\mathcal{S}}_{22}\right) \\
= & \widehat{P}_{2} \widehat{A}+\widehat{A}^{\top} \widehat{P}_{2}+\widehat{C}^{\top} P_{2} \widehat{C}+\widehat{Q}_{2}+\left(\bar{\Theta}^{*}\right)^{\top}\left(\widehat{R}^{2}+\widehat{D}^{\top} P_{2} \widehat{D}\right) \bar{\Theta}^{*} \\
& +\left(\widehat{P}_{2} \widehat{B}+\widehat{C}^{\top} P_{2} \widehat{D}+\widehat{S}_{2}^{\top}\right) \bar{\Theta}^{*}+\left(\bar{\Theta}^{*}\right)^{\top}\left(\widehat{B}^{\top} \widehat{P}_{2}+\widehat{D}^{\top} P_{1} \widehat{C}+\widehat{S}_{2}\right) .
\end{aligned}\right.
$$

This yields (3.35). The proof is complete.

Now, we are ready to present the main result of this subsection, which characterizes the closed-loop Nash equilibrium of Problem (MF-SDG).

Theorem 3.7. Let (H2) hold. Problem (MF-SDG) admits a closed-loop Nash equilibrium $\left(\mathbf{\Theta}^{*}, v^{*}(\cdot)\right)$ if and only if the following statements hold:

(i) The system (3.35) admits a solution pair $\left(P_{i}, \widehat{P}_{i}\right) \in \mathbb{S}^{n} \times \mathbb{S}^{n}$ and (3.36)-(3.37) are satisfied.

(ii) For $i=1,2$, the following BSDE:

$$
\mathrm{d} \eta_{i}(t)=-\left\{A_{\Theta^{*}}^{\top} \eta_{i}(t)+C_{\Theta^{*}}^{\top}\left[\zeta_{i}(t)+P_{i} \sigma(t)\right]+P_{i} b(t)+q_{i}(t)+\left(\Theta^{*}\right)^{\top} \rho_{i}(t)\right\} \mathrm{d} t+\zeta_{i}(t) \mathrm{d} W(t), \quad t \geqslant 0,
$$

admits a solution $\left(\eta_{i}(\cdot), \zeta_{i}(\cdot)\right) \in \mathscr{X}[0, \infty) \times L_{\mathbb{F}}^{2}\left(\mathbb{R}^{n}\right)$ such that

$$
\begin{aligned}
0=( & \left.R_{i i}+D_{i}^{\top} P_{i} D\right)\left(v^{*}(t)-\mathbb{E}\left[v^{*}(t)\right]\right)+B_{i}^{\top}\left(\eta_{i}(t)-\mathbb{E}\left[\eta_{i}(t)\right]\right)+D_{i}^{\top}\left(\zeta_{i}(t)-\mathbb{E}\left[\zeta_{i}(t)\right]\right) \\
& +D_{i}^{\top} P_{i}(\sigma(t)-\mathbb{E}[\sigma(t)])+\rho_{i i}(t)-\mathbb{E}\left[\rho_{i i}(t)\right], \quad \text { a.e. } t \in[0, \infty), \text { a.s., } \quad i=1,2,
\end{aligned}
$$

and the following ODE admits a solution $\bar{\eta}_{i}(\cdot) \in L^{2}\left(\mathbb{R}^{n}\right)$ :

$$
\dot{\bar{\eta}}_{i}(t)+\widehat{A}_{\bar{\Theta}^{*}}^{\top} \bar{\eta}_{i}(t)+\widehat{C}_{\bar{\Theta}^{*}}^{\top}\left(\mathbb{E}\left[\zeta_{i}(t)\right]+P_{i} \mathbb{E}[\sigma(t)]\right)+\widehat{P}_{i} \mathbb{E}[b(t)]+\mathbb{E}\left[q_{i}(t)\right]+\left(\bar{\Theta}^{*}\right)^{\top} \mathbb{E}\left[\rho_{i}(t)\right]=0, \quad t \geqslant 0,
$$

satisfying

$$
\left(\widehat{R}_{i i}+\widehat{D}_{i}^{\top} P_{i} \widehat{D}\right) \mathbb{E}\left[v^{*}(t)\right]+\widehat{B}_{i}^{\top} \bar{\eta}_{i}(t)+\widehat{D}_{i}^{\top} \mathbb{E}\left[\zeta_{i}(t)\right]+\widehat{D}_{i}^{\top} P_{i} \mathbb{E}[\sigma(t)]+\mathbb{E}\left[\rho_{i i}(t)\right]=0, \quad \text { a.e. } t \in[0, \infty) .
$$

Proof. Necessity. Suppose that $\left(\mathbf{\Theta}^{*}, v^{*}(\cdot)\right)$ is a closed-loop Nash equilibrium of Problem (MF-SDG). Then by Proposition 3.6, (i) holds.

For (ii), we first note that system (3.35) is equivalent to

$$
\left\{\begin{array}{l}
P_{i} A_{\Theta^{*}}+A_{\Theta^{*}}^{\top} P_{i}+C_{\Theta^{*}}^{\top} P_{i} C_{\Theta^{*}}+Q_{i}+S_{i}^{\top} \Theta^{*}+\left(\Theta^{*}\right)^{\top} S_{i}+\left(\Theta^{*}\right)^{\top} R_{i} \Theta^{*}=0 \\
\widehat{P}_{i} \widehat{A}_{\bar{\Theta}^{*}}+\widehat{A}_{\bar{\Theta}^{*}}^{\top} \widehat{P}_{i}+\widehat{C}_{\bar{\Theta}^{*}}^{\top} P_{i} \widehat{C}_{\Theta^{*}}+\widehat{Q}_{i}+\widehat{S}_{i}^{\top} \bar{\Theta}^{*}+\left(\bar{\Theta}^{*}\right)^{\top} \widehat{S}_{i}+\left(\bar{\Theta}^{*}\right)^{\top} \widehat{R}_{i} \bar{\Theta}^{*}=0
\end{array}\right.
$$

Let $\left(X^{*}(\cdot), Y_{i}^{*}(\cdot), Z_{i}^{*}(\cdot)\right) \in \mathscr{X}[0, \infty) \times \mathscr{X}[0, \infty) \times L_{\mathbb{F}}^{2}\left(\mathbb{R}^{n}\right)$ be the solution to MF-FBSDE

$$
\left\{\begin{aligned}
& \mathrm{d} X^{*}(t)=\left\{\begin{array}{l}
A_{\Theta^{*}} \\
\end{array} X^{*}+\bar{A}_{\Theta^{*}} \mathbb{E}\left[X^{*}\right]+B v^{*}+\bar{B} \mathbb{E}\left[v^{*}\right]+b\right\} \mathrm{d} t \\
&+\left\{C_{\Theta^{*}} X^{*}+\bar{C}_{\Theta^{*}} \mathbb{E}\left[X^{*}\right]+D v^{*}+\bar{D} \mathbb{E}\left[v^{*}\right]+\sigma\right\} \mathrm{d} W(t), \\
&-\mathrm{d} Y_{i}^{*}(t)=\left\{\begin{aligned}
A_{\Theta^{*}}^{\top} Y_{i}^{*}+\bar{A}_{\Theta^{*}}^{\top} \mathbb{E}\left[Y_{i}^{*}\right]+C_{\Theta^{*}}^{\top} Z_{i}^{*}+\bar{C}_{\Theta^{*}}^{\top} \mathbb{E}\left[Z_{i}^{*}\right]+\mathcal{Q}_{i}^{*} X^{*}+\overline{\mathcal{Q}}_{i}^{*} \mathbb{E}\left[X^{*}\right] \\
\left.\quad+\left(\mathcal{S}_{i}^{*}\right)^{\top} v^{*}+\left(\overline{\mathcal{S}}_{i}^{*}\right)^{\top} \mathbb{E}\left[v^{*}\right]+q_{i}^{*}+\left(\bar{\Theta}^{*}-\Theta^{*}\right)^{\top} \mathbb{E}\left[\rho_{i}\right]\right\} \mathrm{d} t-Z_{i}^{*} \mathrm{~d} W(t), \quad t \geqslant 0,
\end{aligned}\right. \\
& \\
& X^{*}(0)=x, \quad
\end{aligned}\right.
$$


for $i=1,2$. Proceeding as in the proof of Proposition 3.5, we see that $\left(X^{*}(\cdot), Y_{i}^{*}(\cdot), Z_{i}^{*}(\cdot)\right)$ satisfies

$$
\begin{gathered}
B_{i}^{\top} Y_{i}^{*}+\bar{B}_{i}^{\top} \mathbb{E}\left[Y_{i}^{*}\right]+D_{i}^{\top} Z_{i}^{*}+\bar{D}_{i}^{\top} \mathbb{E}\left[Z_{i}^{*}\right]+\mathcal{S}_{i i}^{*} X^{*}+\overline{\mathcal{S}}_{i i}^{*} \mathbb{E}\left[X^{*}\right]+R_{i i} v^{*}+\bar{R}_{i i} \mathbb{E}\left[v^{*}\right]+\rho_{i i}=0, \\
\text { a.e. } t \in[0, \infty), \text { a.s. }
\end{gathered}
$$

where $\mathcal{S}_{i i}^{*} \triangleq S_{i i}+R_{i i} \Theta^{*}, \overline{\mathcal{S}}_{i i}^{*} \triangleq \bar{S}_{i i}+\widehat{R}_{i i} \bar{\Theta}^{*}-R_{i i} \Theta^{*}, i=1,2$. Now, we define

$$
\left\{\begin{array}{l}
\alpha_{i} \triangleq Y_{i}^{*}-\mathbb{E}\left[Y_{i}^{*}\right]-P_{i}\left(X^{*}-\mathbb{E}\left[X^{*}\right]\right), \quad \bar{\eta}_{i} \triangleq \mathbb{E}\left[Y_{i}^{*}\right]-\widehat{P}_{i} \mathbb{E}\left[X^{*}\right], \\
\zeta_{i} \triangleq Z_{i}^{*}-P_{i} C_{\Theta^{*}}\left(X^{*}-\mathbb{E}\left[X^{*}\right]\right)-P_{i} \widehat{C}_{\bar{\Theta}^{*}} \mathbb{E}\left[X^{*}\right]-P_{i} D\left(v^{*}-\mathbb{E}\left[v^{*}\right]\right)-P_{i} \sigma-P_{i} \widehat{D} \mathbb{E}\left[v^{*}\right], \quad i=1,2 .
\end{array}\right.
$$

We want to show that

$$
\alpha_{i}(\cdot)=\eta_{i}(\cdot)-\mathbb{E}\left[\eta_{i}(\cdot)\right]
$$

with $\eta_{i}(\cdot), \zeta_{i}(\cdot), \bar{\eta}(\cdot)$ satisfy $(3.43)-(3.46)$, for $i=1,2$. For this target, applying Itô's formula to $\alpha_{i}(\cdot)$ yields

$$
\begin{aligned}
d \alpha_{i}(t)= & -\left\{A_{\Theta^{*}}^{\top}\left(Y_{i}^{*}-\mathbb{E}\left[Y_{i}^{*}\right]\right)+C_{\Theta^{*}}^{\top}\left(Z_{i}^{*}-\mathbb{E}\left[Z_{i}^{*}\right]\right)+\mathcal{Q}_{i}\left(X^{*}-\mathbb{E}\left[X^{*}\right]\right)+\mathcal{S}_{i}\left(v^{*}-\mathbb{E}\left[v^{*}\right]\right)+q_{i}^{*}-\mathbb{E}\left[q_{i}^{*}\right]\right\} \mathrm{d} t \\
& +Z_{i} d W(t)-P_{i}\left\{\mathcal{A}_{\Theta^{*}}^{\top}\left(X^{*}-\mathbb{E}\left[X^{*}\right]\right)+B\left(v^{*}-\mathbb{E}\left[v^{*}\right]\right)+b-\mathbb{E}[b]\right\} \mathrm{d} t-P_{i}\left\{C_{\Theta^{*}}^{\top}\left(X^{*}-\mathbb{E}\left[X^{*}\right]\right)\right. \\
& \left.+\left(\widehat{C}+\widehat{D} \bar{\Theta}^{*}\right) \mathbb{E}\left[\mathrm{X}^{*}\right]+D\left(v^{*}-\mathbb{E}\left[v^{*}\right]\right)+\widehat{D} \mathbb{E}\left[v^{*}\right]+\sigma\right\} \mathrm{d} W(t) \\
= & -\left\{A_{\Theta^{*}}^{\top} \alpha_{i}+C_{\Theta^{*}}^{\top}\left(\zeta_{i}-\mathbb{E}\left[\zeta_{i}\right]\right)+\left(P_{i} A_{\Theta^{*}}+A_{\Theta^{*}}^{\top} P_{i}+C_{\Theta^{*}}^{\top} P_{i} C_{\Theta^{*}}+\mathcal{Q}_{i}\right)\left(X^{*}-\mathbb{E}\left[X^{*}\right]\right)\right. \\
& +\left[P_{i} B+C^{\top} P_{i} D+S_{i}^{\top}+\left(\Theta^{*}\right)^{\top}\left(R_{i}+D^{\top} P_{i} D\right)\right]\left(v^{*}-\mathbb{E}\left[v^{*}\right]\right)+\mathcal{C}_{\Theta^{*}}^{\top} P_{i}(\sigma-\mathbb{E}[\sigma]) \\
& \left.+P_{i}(b-\mathbb{E}[b])+q_{i}-\mathbb{E}\left[q_{i}\right]+\left(\Theta^{*}\right)^{\top}\left(\rho_{i}-\mathbb{E}\left[\rho_{i}\right]\right)\right\} \mathrm{d} t+\zeta_{i} d W(t) \\
= & -\left\{A_{\Theta^{*}}^{\top} \alpha_{i}+C_{\Theta^{*}}^{\top}\left(\zeta_{i}-\mathbb{E}\left[\zeta_{i}\right]\right)+C_{\Theta^{*}}^{\top} P_{i}(\sigma-\mathbb{E}[\sigma])\right. \\
& \left.+\left(\Theta^{*}\right)^{\top}\left(\rho_{i}-\mathbb{E}\left[\rho_{i}\right]\right)+P_{i}(b-\mathbb{E}[b])+q_{i}-\mathbb{E}\left[q_{i}\right]\right\} \mathrm{d} t+\zeta_{i} d W(t) .
\end{aligned}
$$

Since the solution $\left(\eta_{i}(\cdot), \zeta_{i}(\cdot)\right)$ to $(3.43)$ satisfies

$$
\begin{aligned}
d\left(\eta_{i}(t)-\mathbb{E}\left[\eta_{i}(t)\right]\right)=- & \left\{A_{\Theta^{*}}^{\top}\left(\eta_{i}-\mathbb{E}\left[\eta_{i}\right]\right)+C_{\Theta^{*}}^{\top}\left(\zeta_{i}-\mathbb{E}\left[\zeta_{i}\right]\right)+C_{\Theta^{*}}^{\top} P_{i}(\sigma-\mathbb{E}[\sigma])\right. \\
& \left.+\left(\Theta^{*}\right)^{\top}\left(\rho_{i}-\mathbb{E}\left[\rho_{i}\right]\right)+P_{i}(b-\mathbb{E}[b])+q_{i}-\mathbb{E}\left[q_{i}\right]\right\} \mathrm{d} t+\zeta_{i} d W(t), \quad t \geqslant 0,
\end{aligned}
$$

by the uniqueness of solutions, we obtain (3.51). Moreover, we get

$$
\begin{aligned}
- & \dot{\eta}_{i}(t)=\left[\widehat{A}+\widehat{B} \bar{\Theta}^{*}\right]^{\top} \mathbb{E}\left[Y_{i}^{*}\right]+\left[\widehat{C}+\widehat{D} \bar{\Theta}^{*}\right]^{\top} \mathbb{E}\left[Z_{i}^{*}\right]+\widehat{\mathcal{Q}}_{i} \mathbb{E}\left[X^{*}\right] \\
& +\widehat{\mathcal{S}}_{i} \mathbb{E}\left[v^{*}\right]+\mathbb{E}\left[q_{i}^{*}\right]+\widehat{P}_{i}\left[\widehat{A}+\widehat{B} \bar{\Theta}^{*}\right] \mathbb{E}\left[X^{*}\right]+\widehat{P}_{i} \widehat{B} \mathbb{E}\left[v^{*}\right]+\widehat{P}_{i} \mathbb{E}[b] \\
= & {\left[\widehat{A}+\widehat{B} \bar{\Theta}^{*}\right]^{\top} \bar{\eta}_{i}+\left\{\widehat{P}_{i}\left(\widehat{A}+\widehat{B} \bar{\Theta}^{*}\right)+\left(\widehat{A}+\widehat{B} \bar{\Theta}^{*}\right)^{\top} \widehat{P}_{i}+\left[\widehat{C}+\widehat{D} \Theta^{*}\right]^{\top} P_{i}\left[\widehat{C}+\widehat{D} \Theta^{*}\right]+\widehat{Q}_{i}\right.} \\
& \left.+\widehat{S}_{i}^{\top} \bar{\Theta}^{*}+\left(\bar{\Theta}^{*}\right)^{\top} \widehat{S}_{i}+\left(\bar{\Theta}^{*}\right)^{\top} \widehat{R}_{i} \bar{\Theta}^{*}\right\} \mathbb{E}\left[X^{*}\right]+\left\{\widehat{P}_{i} \widehat{B}^{\top} \widehat{C}^{\top} P_{i} \widehat{D}+\widehat{S}_{i}^{\top}+\left(\bar{\Theta}^{*}\right)^{\top}\left[\widehat{R}_{i}+\widehat{D}^{\top} P_{i} \widehat{D}\right]\right\} \mathbb{E}\left[v^{*}\right] \\
& +\left(\bar{\Theta}^{*}\right)^{\top}\left[\widehat{D}^{\top}\left(P_{i} \mathbb{E}[\sigma]+\mathbb{E}\left[\zeta_{i}\right]\right)+\mathbb{E}\left[\rho_{i}\right]\right]+\widehat{C}^{\top}\left(P_{i} \mathbb{E}[\sigma]+\mathbb{E}\left[\zeta_{i}\right]\right)+\widehat{P}_{i} \mathbb{E}[b]+\mathbb{E}\left[q_{i}\right] \\
= & \left(\widehat{A}+\widehat{B} \bar{\Theta}^{*}\right)^{\top} \bar{\eta}_{i}+\left(\bar{\Theta}^{*}\right)^{\top}\left[\widehat{D}^{\top}\left(P_{i} \mathbb{E}[\sigma]+\mathbb{E}\left[\zeta_{i}\right]\right)+\mathbb{E}\left[\rho_{i}\right]\right]+\widehat{C}^{\top}\left(P_{i} \mathbb{E}[\sigma]+\mathbb{E}\left[\zeta_{i}\right]\right)+\widehat{P}_{i} \mathbb{E}[b]+\mathbb{E}\left[q_{i}\right],
\end{aligned}
$$

which is (3.45). Further, from (3.49) we have

$$
\widehat{R}_{i i} \mathbb{E}\left[v^{*}\right]+\widehat{B}_{i}^{\top} \mathbb{E}\left[Y_{i}^{*}\right]+\widehat{D}_{i}^{\top} \mathbb{E}\left[Z_{i}^{*}\right]+\left[\widehat{S}_{i i}+\widehat{R}_{i i} \bar{\Theta}^{*}\right] \mathbb{E}\left[X^{*}\right]+\mathbb{E}\left[\rho_{i i}\right]=0, \quad \text { a.e.t } \in[0, \infty), i=1,2,
$$


and

$$
\begin{gathered}
R_{i i}\left(v^{*}-\mathbb{E}\left[v^{*}\right]\right)+B_{i}^{\top}\left(Y_{i}^{*}-\mathbb{E}\left[Y_{i}^{*}\right]\right)+D_{i}^{\top}\left(Z_{i}^{*}-\mathbb{E}\left[Z_{i}^{*}\right]\right)+\left(S_{i i}+R_{i i} \Theta^{*}\right)\left(X^{*}-\mathbb{E}\left[X^{*}\right]\right)+\rho_{i i}-\mathbb{E}\left[\rho_{i i}\right]=0, \\
\text { a.e. } t \in[0, \infty) \text {, a.s., } i=1,2 .
\end{gathered}
$$

Now, (3.36), (3.50) and (3.54) yields

$$
\begin{aligned}
0= & \widehat{R}_{i i} \mathbb{E}\left[v^{*}\right]+\widehat{B}_{i}^{\top}\left(\bar{\eta}_{i}+\widehat{P}_{i} \mathbb{E}\left[X^{*}\right]\right)+\widehat{D}_{i}^{\top}\left\{\mathbb{E}\left[\zeta_{i}\right]+P_{i} \mathbb{E}[\sigma]+P_{i} \widehat{C} \mathbb{E}\left[X^{*}\right]+P_{i} \widehat{D} \mathbb{E}\left[v^{*}\right]\right\}+\left[\widehat{S}_{i i}+\widehat{R}_{i i} \bar{\Theta}^{*}\right] \mathbb{E}\left[X^{*}\right]+\mathbb{E}\left[\rho_{i i}\right] \\
= & \left(\widehat{R}_{i i}+\widehat{D}_{i}^{\top} P_{i} \widehat{D}\right) \mathbb{E}\left[v^{*}\right]+\left\{\widehat{B}_{i}^{\top} \widehat{P}_{i}+\widehat{D}_{i}^{\top} P_{i} \widehat{C}+\widehat{S}_{i i}+\left(\widehat{R}_{i i}+\widehat{D}_{i}^{\top} P_{i} \widehat{D}\right) \Theta^{*}\right\} \mathbb{E}\left[X^{*}\right] \\
& +\widehat{B}_{i}^{\top} \bar{\eta}_{i}+\widehat{D}_{i}^{\top}\left(\mathbb{E}\left[\zeta_{i}\right]+P_{i} \mathbb{E}[\sigma]\right)+\mathbb{E}\left[\rho_{i i}\right] \\
= & \left(\widehat{R}_{i i}+\widehat{D}_{i}^{\top} P_{i} \widehat{D}\right) \mathbb{E}\left[v^{*}\right]+\widehat{B}_{i}^{\top} \bar{\eta}_{i}+\widehat{D}_{i}^{\top}\left(\mathbb{E}\left[\zeta_{i}\right]+P_{i} \mathbb{E}[\sigma]\right)+\mathbb{E}\left[\rho_{i i}\right], \quad \text { a.e. } t \in[0, \infty), i=1,2 .
\end{aligned}
$$

Thus (3.46) holds. Furthermore, (3.36), (3.50) and (3.55) yields

$$
\begin{aligned}
& 0= R_{i i}\left(v^{*}-\mathbb{E}\left[v^{*}\right]\right)+B_{i}^{\top}\left[\alpha_{i}+P_{i}\left(X^{*}-\mathbb{E}\left[X^{*}\right]\right)\right]+D_{i}^{\top}\left\{\zeta_{i}-\mathbb{E}\left[\zeta_{i}\right]+P_{i} C_{\Theta^{*}}\left(X^{*}-\mathbb{E}\left[X^{*}\right]\right)\right. \\
&\left.+P_{i} D\left(v^{*}-\mathbb{E}\left[v^{*}\right]\right)+P_{i}(\sigma-\mathbb{E}[\sigma])\right\}+\left(S_{i i}+R_{i i} \Theta^{*}\right)\left(X^{*}-\mathbb{E}\left[X^{*}\right]\right)+\rho_{i i}-\mathbb{E}\left[\rho_{i i}\right] \\
&=\left(R_{i i}+D_{i}^{\top} P_{i} D\right)\left(v^{*}-\mathbb{E}\left[v^{*}\right]\right)+B_{i}^{\top} \alpha_{i}+D_{i}^{\top}\left(\zeta_{i}-\mathbb{E}\left[\zeta_{i}\right]\right)+D_{i}^{\top} P_{i}(\sigma-\mathbb{E}[\sigma]) \\
&+\left[B_{i}^{\top} P_{i}+D_{i}^{\top} P_{i} C+S_{i i}+\left(R_{i i}+D_{i}^{\top} P_{i} D\right) \Theta^{*}\right]\left(X^{*}-\mathbb{E}\left[X^{*}\right]\right)+\rho_{i i}-\mathbb{E}\left[\rho_{i i}\right] \\
&=\left(R_{i i}+D_{i}^{\top} P_{i} D\right)\left(v^{*}-\mathbb{E}\left[v^{*}\right]\right)+B_{i}^{\top} \alpha_{i}+D_{i}^{\top}\left(\zeta_{i}-\mathbb{E}\left[\zeta_{i}\right]\right)+D_{i}^{\top} P_{i}(\sigma-\mathbb{E}[\sigma])+\rho_{i i}-\mathbb{E}\left[\rho_{i i}\right], \\
& \text { a.e. } t \in[0, \infty), \text { a.s., } i=1,2 .
\end{aligned}
$$

Hence, by (3.56), we get (3.44).

Sufficiency. We choose any $x \in \mathbb{R}^{n}$ and $v(\cdot) \equiv\left(\begin{array}{c}v_{1}(\cdot) \\ v_{2}(\cdot)\end{array}\right) \in L_{\mathbb{F}}^{2}\left(\mathbb{R}^{m}\right)$. Denote $w(\cdot) \equiv\left(\begin{array}{c}v_{1}(\cdot) \\ v_{2}^{*}(\cdot)\end{array}\right)$ and let $X^{w}(\cdot) \equiv$ $X\left(\cdot ; x, \boldsymbol{\Theta}_{1}^{*}, v_{1}(\cdot) ; \boldsymbol{\Theta}_{2}^{*}, v_{2}^{*}(\cdot)\right)$ be the solution to the state equation

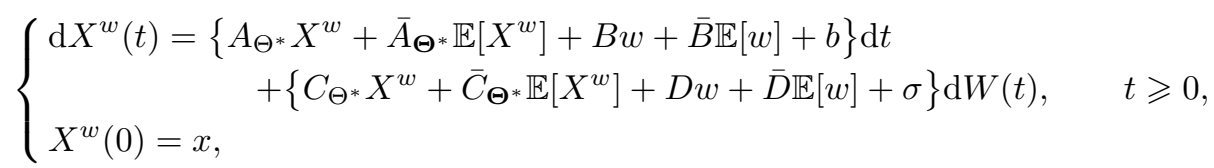

corresponding to $x$ and $\left(\boldsymbol{\Theta}_{1}^{*}, v_{1}(\cdot), \boldsymbol{\Theta}_{2}^{*}, v_{2}^{*}(\cdot)\right)$. Also, we have

$$
\begin{aligned}
J_{1}\left(x ; \Theta^{*}\right. & \left.\left(X^{w}(\cdot)-\mathbb{E}\left[X^{w}(\cdot)\right]\right)+\bar{\Theta}^{*} \mathbb{E}\left[X^{w}(\cdot)\right]+w(\cdot)\right) \\
=\mathbb{E} \int_{0}^{\infty} & {\left[\left\langle\left(Q_{1}+S_{1}^{\top} \Theta^{*}+\left(\Theta^{*}\right)^{\top} S_{1}+\left(\Theta^{*}\right)^{\top} R_{1} \Theta^{*}\right)\left(X^{w}-\mathbb{E}\left[X^{w}\right]\right), X^{w}-\mathbb{E}\left[X^{w}\right]\right\rangle\right.} \\
& +2\left\langle\left(S_{1}+R_{1} \Theta^{*}\right)^{\top}(w-\mathbb{E}[w]), X^{w}-\mathbb{E}\left[X^{w}\right]\right\rangle+\left\langle R_{1}(w-\mathbb{E}[w]), w-\mathbb{E}[w]\right\rangle \\
& +2\left\langle q_{1}+\left(\Theta^{*}\right)^{\top} \rho_{1}, X^{w}-\mathbb{E}\left[X^{w}\right]\right\rangle+2\left\langle\rho_{1}, w-\mathbb{E}[w]\right\rangle \\
& \left.\left.+\left\langle\widehat{Q}_{1}+\widehat{S}_{1}^{\top} \bar{\Theta}^{*}+\left(\bar{\Theta}^{*}\right)^{\top} \widehat{S}_{1}+\left(\bar{\Theta}^{*}\right)^{\top} \widehat{R}_{1} \bar{\Theta}^{*}\right) \mathbb{E}\left[X^{w}\right], \mathbb{E}\left[X^{w}\right]\right\rangle+\left\langle\widehat{S}_{1}+\widehat{R}_{1} \bar{\Theta}^{*}\right)^{\top} \mathbb{E}[w], \mathbb{E}\left[X^{w}\right]\right\rangle \\
& \left.+\left\langle\widehat{R}_{1} \mathbb{E}[w], \mathbb{E}[w]\right\rangle+2\left\langle\mathbb{E}\left[q_{1}\right]+\left(\bar{\Theta}^{*}\right)^{\top} \mathbb{E}\left[\rho_{1}\right], \mathbb{E}\left[X^{w}\right]\right\rangle+2\left\langle\mathbb{E}\left[\rho_{1}\right], \mathbb{E}[w]\right\rangle\right] \mathrm{d} t .
\end{aligned}
$$

Applying Itô's formula to

$$
\left\langle P_{1}\left(X^{w}(\cdot)-\mathbb{E}\left[X^{w}(\cdot)\right]\right)+2 \eta_{1}(\cdot), X^{w}(\cdot)-\mathbb{E}\left[X^{w}(\cdot)\right]\right\rangle+\left\langle\widehat{P}_{1} \mathbb{E}\left[X^{w}(\cdot)\right]+2 \bar{\eta}_{1}(\cdot), \mathbb{E}\left[X^{w}(\cdot)\right]\right\rangle
$$


we have

$$
\begin{aligned}
- & \left\langle\widehat{P}_{1} x+2 \bar{\eta}_{1}(0), x\right\rangle=\mathbb{E} \int_{0}^{\infty}\left[\left\langle\left(P_{1} A_{\Theta^{*}}+A_{\Theta^{*}}^{\top} P_{1}+C_{\Theta^{*}}^{\top} P_{1} C_{\Theta^{*}}\right)\left(X^{w}-\mathbb{E}\left[X^{w}\right]\right), X^{w}-\mathbb{E}\left[X^{w}\right]\right\rangle\right. \\
& +2\left\langle\left(P_{1} B+C_{\Theta^{*}}^{\top} P_{1} D\right)(w-\mathbb{E}[w]), X^{w}-\mathbb{E}\left[X^{w}\right]\right\rangle+\left\langle D^{\top} P_{1} D(w-\mathbb{E}[w]), w-\mathbb{E}[w]\right\rangle \\
& -2\left\langle\left(\Theta^{*}\right)^{\top} \rho_{1}+q_{1}, X^{w}-\mathbb{E}\left[X^{w}\right]\right\rangle+2\left\langle B^{\top} \eta_{1}+D^{\top} \zeta_{1}+D^{\top} P_{1} \sigma, w-\mathbb{E}[w]\right\rangle \\
& +\left\langle\left(\widehat{P}_{1} \widehat{A}_{\bar{\Theta}^{*}}+\widehat{A}_{\bar{\Theta}^{*}}^{\top} \widehat{P}_{1}+\widehat{C}_{\bar{\Theta}^{*}}^{\top} P_{1} \widehat{C}_{\bar{\Theta}^{*}}\right) \mathbb{E}\left[X^{w}\right], \mathbb{E}\left[X^{w}\right]\right\rangle+2\left\langle\left(\widehat{P}_{1} \widehat{B}+\widehat{C}_{\bar{\Theta}^{*}}^{\top} P_{1} \widehat{D}\right) \mathbb{E}[w], \mathbb{E}\left[X^{w}\right]\right\rangle \\
& +\left\langle\widehat{D}^{\top} P_{1} \widehat{D} \mathbb{E}[w], \mathbb{E}[w]\right\rangle-2\left\langle\left(\bar{\Theta}^{*}\right)^{\top} \mathbb{E}\left[\rho_{1}\right]+\mathbb{E}\left[q_{1}\right], \mathbb{E}\left[X^{w}\right]\right\rangle+2\left\langle\widehat{B}^{\top} \bar{\eta}_{1}+\widehat{D}^{\top}\left(P_{1} \mathbb{E}[\sigma]+\mathbb{E}\left[\zeta_{1}\right]\right), \mathbb{E}[w]\right\rangle \\
& \left.+\left\langle P_{1} \sigma, \sigma\right\rangle+2\left\langle\eta_{1}, b-\mathbb{E}[b]\right\rangle+2\left\langle\zeta_{1}, \sigma\right\rangle+2\left\langle\bar{\eta}_{1}, \mathbb{E}[b]\right\rangle\right] \mathrm{d} t .
\end{aligned}
$$

Combining the above two equalities, together with conditions (3.44) and (3.46), we obtain

$$
\begin{aligned}
& J_{1}\left(x ; \Theta^{*}\left(X^{w}(\cdot)-\mathbb{E}\left[X^{w}(\cdot)\right]\right)+\bar{\Theta}^{*} \mathbb{E}\left[X^{w}(\cdot)\right]+w(\cdot)\right)-\left\langle\widehat{P}_{1} x+2 \bar{\eta}_{1}(0), x\right\rangle \\
& =\mathbb{E} \int_{0}^{\infty}\left[\left\langle\left(R_{1}+D^{\top} P_{1} D\right)(w-\mathbb{E}[w]), w-\mathbb{E}[w]\right\rangle+2\left\langle B^{\top} \eta_{1}+D^{\top} \zeta_{1}+D^{\top} P_{1} \sigma+\rho_{1}, w-\mathbb{E}[w]\right\rangle\right. \\
& +\left\langle\left[\widehat{R}_{1}+\widehat{D}^{\top} P_{1} \widehat{D}\right] \mathbb{E}[w], \mathbb{E}[w]\right\rangle+2\left\langle\widehat{B}^{\top} \bar{\eta}_{1}+\widehat{D}^{\top}\left(P_{1} \mathbb{E}[\sigma]+\mathbb{E}\left[\zeta_{1}\right]\right)+\mathbb{E}\left[\rho_{1}\right], \mathbb{E}[w]\right\rangle \\
& \left.+\left\langle P_{1} \sigma, \sigma\right\rangle+2\left\langle\eta_{1}, b-\mathbb{E}[b]\right\rangle+2\left\langle\zeta_{1}, \sigma\right\rangle+2\left\langle\bar{\eta}_{1}, \mathbb{E}[b]\right\rangle\right] \mathrm{d} t \\
& =\mathbb{E} \int_{0}^{\infty}\left[\left\langle\left(R_{111}+D_{1}^{\top} P_{1} D_{1}\right)\left(v_{1}-\mathbb{E}\left[v_{1}\right]\right), v_{1}-\mathbb{E}\left[v_{1}\right]\right\rangle+2\left\langle\left(R_{112}+D_{1}^{\top} P_{1} D_{2}\right)\left(v_{2}^{*}-\mathbb{E}\left[v_{2}^{*}\right]\right), v_{1}-\mathbb{E}\left[v_{1}\right]\right\rangle\right. \\
& +\left\langle\left(R_{122}+D_{2}^{\top} P_{1} D_{2}\right)\left(v_{2}^{*}-\mathbb{E}\left[v_{2}^{*}\right]\right), v_{2}^{*}-\mathbb{E}\left[v_{2}^{*}\right]\right\rangle \\
& +2\left\langle B_{1}^{\top}\left(\eta_{1}-\mathbb{E}\left[\eta_{1}\right]\right)+D_{1}^{\top}\left(\zeta_{1}-\mathbb{E}\left[\zeta_{1}\right]\right)+D_{1}^{\top} P_{1}(\sigma-\mathbb{E}[\sigma])+\rho_{11}-\mathbb{E}\left[\rho_{11}\right], v_{1}-\mathbb{E}\left[v_{1}\right]\right\rangle \\
& +2\left\langle B_{2}^{\top}\left(\eta_{1}-\mathbb{E}\left[\eta_{1}\right]\right)+D_{2}^{\top}\left(\zeta_{1}-\mathbb{E}\left[\zeta_{1}\right]\right)+D_{2}^{\top} P_{1}(\sigma-\mathbb{E}[\sigma])+\rho_{12}-\mathbb{E}\left[\rho_{12}\right], v_{2}^{*}-\mathbb{E}\left[v_{2}^{*}\right]\right\rangle \\
& +\left\langle\left(\widehat{R}_{111}+\widehat{D}_{1}^{\top} P_{1} \widehat{D}_{1}\right) \mathbb{E}\left[v_{1}\right], \mathbb{E}\left[v_{1}\right]\right\rangle+2\left\langle\left(\widehat{R}_{112}+\widehat{D}_{1}^{\top} P_{1} \widehat{D}_{2}\right) \mathbb{E}\left[v_{2}^{*}\right], \mathbb{E}\left[v_{1}\right]\right\rangle \\
& +\left\langle\left(\widehat{R}_{122}+\widehat{D}_{2}^{\top} P_{1} \widehat{D}_{2}\right) \mathbb{E}\left[v_{2}^{*}\right], \mathbb{E}\left[v_{2}^{*}\right]\right\rangle+2\left\langle\widehat{B}_{1}^{\top} \bar{\eta}_{1}+\widehat{D}_{1}^{\top}\left(P_{1} \mathbb{E}[\sigma]+\mathbb{E}\left[\zeta_{1}\right]\right)+\mathbb{E}\left[\rho_{11}\right], \mathbb{E}\left[v_{1}\right]\right\rangle \\
& +2\left\langle\widehat{B}_{2}^{\top} \bar{\eta}_{1}+\widehat{D}_{2}^{\top}\left(P_{1} \mathbb{E}[\sigma]+\mathbb{E}\left[\zeta_{1}\right]\right)+\mathbb{E}\left[\rho_{12}\right], \mathbb{E}\left[v_{2}^{*}\right]\right\rangle \\
& \left.+\left\langle P_{1} \sigma, \sigma\right\rangle+2\left\langle\eta_{1}, b-\mathbb{E}[b]\right\rangle+2\left\langle\zeta_{1}, \sigma\right\rangle+2\left\langle\bar{\eta}_{1}, \mathbb{E}[b]\right\rangle\right] \mathrm{d} t \\
& =\mathbb{E} \int_{0}^{\infty}\left[\left\langle\left(R_{111}+D_{1}^{\top} P_{1} D_{1}\right)\left[v_{1}-\mathbb{E}\left[v_{1}\right]-\left(v_{1}^{*}-\mathbb{E}\left[v_{1}^{*}\right]\right)\right], v_{1}-\mathbb{E}\left[v_{1}\right]-\left(v_{1}^{*}-\mathbb{E}\left[v_{1}^{*}\right]\right)\right\rangle\right. \\
& -\left\langle\left(R_{111}+D_{1}^{\top} P_{1} D_{1}\right)\left(v_{1}^{*}-\mathbb{E}\left[v_{1}^{*}\right]\right), v_{1}^{*}-\mathbb{E}\left[v_{1}^{*}\right]\right\rangle+\left\langle\left(R_{122}+D_{2}^{\top} P_{1} D_{2}\right)\left(v_{2}^{*}-\mathbb{E}\left[v_{2}^{*}\right]\right), v_{2}^{*}-\mathbb{E}\left[v_{2}^{*}\right]\right\rangle \\
& +2\left\langle B_{2}^{\top}\left(\eta_{1}-\mathbb{E}\left[\eta_{1}\right]\right)+D_{2}^{\top}\left(\zeta_{1}-\mathbb{E}\left[\zeta_{1}\right]\right)\right. \\
& \left.+D_{2}^{\top} \mathrm{P}_{1}(\sigma-\mathbb{E}[\sigma])+\rho_{12}-\mathbb{E}\left[\rho_{12}\right], v_{2}^{*}-\mathbb{E}\left[v_{2}^{*}\right]\right\rangle \\
& +\left\langle\left[\widehat{R}_{111}+\widehat{D}_{1}^{\top} P_{1} \widehat{D}_{1}\right]\left(\mathbb{E}\left[v_{1}\right]-\mathbb{E}\left[v_{1}^{*}\right]\right), \mathbb{E}\left[v_{1}\right]-\mathbb{E}\left[v_{1}^{*}\right]\right\rangle-\left\langle\left[\widehat{R}_{111}+\widehat{D}_{1}^{\top} P_{1} \widehat{D}_{1}\right] \mathbb{E}\left[v_{1}^{*}\right], \mathbb{E}\left[v_{1}^{*}\right]\right\rangle \\
& \left.+\left\langle\left[\widehat{R}_{122}+\widehat{D}_{2}^{\top} P_{1} \widehat{D}_{2}\right] \mathbb{E}\left[v_{2}^{*}\right], \mathbb{E}\left[v_{2}^{*}\right]\right\rangle+2\left\langle\widehat{B}_{2}^{\top} \bar{\eta}_{1}+\widehat{D}_{2}^{\top} P_{1} \mathbb{E}[\sigma]+\mathbb{E}\left[\zeta_{1}\right]\right)+\mathbb{E}\left[\rho_{12}\right], \mathbb{E}\left[v_{2}^{*}\right]\right\rangle \\
& \left.+\left\langle P_{1} \sigma, \sigma\right\rangle+2\left\langle\eta_{1}, b-\mathbb{E}[b]\right\rangle+2\left\langle\zeta_{1}, \sigma\right\rangle+2\left\langle\bar{\eta}_{1}, \mathbb{E}[b]\right\rangle\right] \mathrm{d} t .
\end{aligned}
$$

Consequently, one gets

$$
\begin{aligned}
J_{1}\left(x ; \Theta^{*}\left\{X^{w}(\cdot)-\mathbb{E}\left[X^{w}(\cdot)\right]\right\}+\bar{\Theta}^{*} \mathbb{E}\left[X^{w}(\cdot)\right]+w(\cdot)\right)-J_{1}\left(x ; \Theta^{*}\left\{X^{*}(\cdot)-\mathbb{E}\left[X^{*}(\cdot)\right]\right\}+\bar{\Theta}^{*} \mathbb{E}\left[X^{*}(\cdot)\right]+v^{*}(\cdot)\right) \\
=\mathbb{E} \int_{0}^{\infty}\left[\left\langle\left(R_{111}+D_{1}^{\top} P_{1} D_{1}\right)\left[v_{1}-\mathbb{E}\left[v_{1}\right]-\left(v_{1}^{*}-\mathbb{E}\left[v_{1}^{*}\right]\right)\right], v_{1}-\mathbb{E}\left[v_{1}\right]-\left(v_{1}^{*}-\mathbb{E}\left[v_{1}^{*}\right]\right)\right\rangle\right. \\
\left.+\left\langle\left[\widehat{R}_{111}+\widehat{D}_{1}^{\top} P_{1} \widehat{D}_{1}\right]\left(\mathbb{E}\left[v_{1}\right]-\mathbb{E}\left[v_{1}^{*}\right]\right), \mathbb{E}\left[v_{1}\right]-\mathbb{E}\left[v_{1}^{*}\right]\right\rangle\right] \mathrm{d} t \geqslant 0,
\end{aligned}
$$


since (3.37) holds with $i=1$.

Similarly, by $(3.37)$ with $i=2$, for any $\bar{w}(\cdot) \equiv\left(v_{1}^{*}(\cdot), v_{2}(\cdot)\right)$ and $X^{\bar{w}}(\cdot) \equiv X\left(\cdot ; x, \boldsymbol{\Theta}_{1}^{*}, v_{1}^{*}(\cdot) ; \boldsymbol{\Theta}_{2}^{*}, v_{2}(\cdot)\right)$, we can prove that the following holds:

$$
\begin{aligned}
J_{2}\left(x ; \Theta^{*}\left\{X^{\bar{w}}(\cdot)-\mathbb{E}\left[X^{\bar{w}}(\cdot)\right]\right\}+\bar{\Theta}^{*} \mathbb{E}\left[X^{\bar{w}}(\cdot)\right]+\bar{w}(\cdot)\right)-J_{2}\left(x ; \Theta^{*}\left\{X^{*}(\cdot)-\mathbb{E}\left[X^{*}(\cdot)\right]\right\}+\bar{\Theta}^{*} \mathbb{E}\left[X^{*}(\cdot)\right]+v^{*}(\cdot)\right) \\
=\mathbb{E} \int_{0}^{\infty}\left[\left\langle\left(R_{222}+D_{2}^{\top} \mathrm{P}_{2} D_{2}\right)\left[v_{2}-\mathbb{E}\left[v_{2}\right]-\left(v_{2}^{*}-\mathbb{E}\left[v_{2}^{*}\right]\right)\right], v_{2}-\mathbb{E}\left[v_{2}\right]-\left(v_{2}^{*}-\mathbb{E}\left[v_{2}^{*}\right]\right)\right\rangle\right. \\
\left.+\left\langle\left[\widehat{R}_{222}+\widehat{D}_{2}^{\top} P_{2} \widehat{D}_{2}\right]\left(\mathbb{E}\left[v_{2}\right]-\mathbb{E}\left[v_{2}^{*}\right]\right), \mathbb{E}\left[v_{2}\right]-\mathbb{E}\left[v_{2}^{*}\right]\right\rangle\right] \mathrm{d} t \geqslant 0 .
\end{aligned}
$$

By Definition 3.2, this proves the sufficiency. The proof is complete.

To conclude this section, let us rewrite system (3.35) in a more compact form so that one can see an interesting feature of it. We define

$$
\mathbf{P} \triangleq\left(\begin{array}{cc}
P_{1} & 0 \\
0 & P_{2}
\end{array}\right), \quad \widehat{\mathbf{P}} \triangleq\left(\begin{array}{cc}
\widehat{P}_{1} & 0 \\
0 & \widehat{P}_{2}
\end{array}\right)
$$

Note that (3.36) is equivalent to (recalling the notation introduced in (3.21) and (3.24))

$$
0=\left(\begin{array}{l}
B_{1}^{\top} P_{1}+D_{1}^{\top} P_{1} C+S_{11} \\
B_{2}^{\top} P_{2}+D_{2}^{\top} P_{2} C+S_{22}
\end{array}\right)+\left(\begin{array}{l}
R_{11}+D_{1}^{\top} P_{1} D \\
R_{22}+D_{2}^{\top} P_{2} D
\end{array}\right) \Theta^{*} \equiv \mathbf{J}^{\top}\left(\mathbf{B}^{\top} \mathbf{P}+\mathbf{D}^{\top} \mathbf{P C}+\mathbf{S}\right) \mathbf{I}_{n}+\mathbf{\Sigma} \Theta^{*},
$$

and

$$
0=\left(\begin{array}{l}
\widehat{B}_{1}^{\top} \widehat{P}_{1}+\widehat{D}_{1}^{\top} P_{1} \widehat{C}+\widehat{S}_{11} \\
\widehat{B}_{2}^{\top} \widehat{P}_{2}+\widehat{D}_{2}^{\top} P_{2} \widehat{C}+\widehat{S}_{22}
\end{array}\right)+\left(\begin{array}{l}
\widehat{R}_{11}+\widehat{D}_{1}^{\top} P_{1} \widehat{D} \\
\widehat{R}_{22}+\widehat{D}_{2}^{\top} P_{2} \widehat{D}
\end{array}\right) \bar{\Theta}^{*} \equiv \mathbf{J}^{\top}\left(\widehat{\mathbf{B}}^{\top} \widehat{\mathbf{P}}+\widehat{\mathbf{D}}^{\top} \mathbf{P} \widehat{\mathbf{C}}+\widehat{\mathbf{S}}\right) \mathbf{I}_{n}+\overline{\mathbf{\Sigma}} \bar{\Theta}^{*},
$$

where

$$
\boldsymbol{\Sigma} \triangleq \mathbf{J}^{\top}\left(\mathbf{R}+\mathbf{D}^{\top} \mathbf{P D}\right) \mathbf{I}_{m} \equiv\left(\begin{array}{l}
R_{11}+D_{1}^{\top} P_{1} D \\
R_{22}+D_{2}^{\top} P_{2} D
\end{array}\right), \quad \overline{\mathbf{\Sigma}} \triangleq \mathbf{J}^{\top}\left(\widehat{\mathbf{R}}+\widehat{\mathbf{D}}^{\top} \mathbf{P} \widehat{\mathbf{D}}\right) \mathbf{I}_{m} \equiv\left(\begin{array}{l}
\widehat{R}_{11}+\widehat{D}_{1}^{\top} P_{1} \widehat{D} \\
\widehat{R}_{22}+\widehat{D}_{2}^{\top} P_{2} \widehat{D}
\end{array}\right) \in \mathbb{R}^{m \times m}
$$

If we assume both $\boldsymbol{\Sigma}$ and $\overline{\boldsymbol{\Sigma}}$ are invertible, then we have

$$
\Theta^{*}=-\boldsymbol{\Sigma}^{-1} \mathbf{J}^{\top}\left(\mathbf{B}^{\top} \mathbf{P}+\mathbf{D}^{\top} \mathbf{P} \mathbf{C}+\mathbf{S}\right) \mathbf{I}_{n}, \quad \bar{\Theta}^{*}=-\overline{\boldsymbol{\Sigma}}^{-1} \mathbf{J}^{\top}\left(\widehat{\mathbf{B}}^{\top} \widehat{\mathbf{P}}+\widehat{\mathbf{D}}^{\top} \mathbf{P} \widehat{\mathbf{C}}+\widehat{\mathbf{S}}\right) \mathbf{I}_{n} \in \mathbb{R}^{m \times n} .
$$

On the other hand, (3.35) can be written as

$$
\left\{\begin{aligned}
\mathbf{P A} & +\mathbf{A}^{\top} \mathbf{P}+\mathbf{C}^{\top} \mathbf{P C}+\mathbf{Q}+\left(\boldsymbol{\Phi}^{*}\right)^{\top}\left(\mathbf{R}+\mathbf{D}^{\top} \mathbf{P} \mathbf{D}\right) \boldsymbol{\Phi}^{*} \\
& +\left(\mathbf{P B}+\mathbf{C}^{\top} \mathbf{P D}+\mathbf{S}^{\top}\right) \mathbf{\Phi}^{*}+\left(\boldsymbol{\Phi}^{*}\right)^{\top}\left(\mathbf{B}^{\top} \mathbf{P}+\mathbf{D}^{\top} \mathbf{P C}+\mathbf{S}\right)=0 \\
\widehat{\mathbf{P}} \widehat{\mathbf{A}}+ & \widehat{\mathbf{A}}^{\top} \widehat{\mathbf{P}}+\widehat{\mathbf{C}}^{\top} \mathbf{P} \widehat{\mathbf{C}}+\widehat{\mathbf{Q}}+\left(\overline{\mathbf{\Phi}}^{*}\right)^{\top}\left(\widehat{\mathbf{R}}+\widehat{\mathbf{D}}^{\top} \mathbf{P} \widehat{\mathbf{D}}\right) \overline{\mathbf{\Phi}}^{*} \\
& +\left(\widehat{\mathbf{P}} \widehat{\mathbf{B}}+\widehat{\mathbf{C}}^{\top} \mathbf{P} \widehat{\mathbf{D}}+\widehat{\mathbf{S}}^{\top}\right) \overline{\boldsymbol{\Phi}}^{*}+\left(\overline{\boldsymbol{\Phi}}^{*}\right)^{\top}\left(\widehat{\mathbf{B}}^{\top} \widehat{\mathbf{P}}+\widehat{\mathbf{D}}^{\top} \mathbf{P} \widehat{\mathbf{C}}+\widehat{\mathbf{S}}\right)=0
\end{aligned}\right.
$$

with

$$
\boldsymbol{\Phi}^{*} \triangleq\left(\begin{array}{cc}
\Theta^{*} & 0 \\
0 & \Theta^{*}
\end{array}\right), \quad \overline{\boldsymbol{\Phi}}^{*} \triangleq\left(\begin{array}{cc}
\bar{\Theta}^{*} & 0 \\
0 & \bar{\Theta}^{*}
\end{array}\right)
$$


Clearly, both equations in (3.61) are symmetric with solutions $\mathbf{P}, \widehat{\mathbf{P}} \in \mathbb{S}^{2 n}$. Recall that system (3.26) (or, equivalently, (3.32)) for closed-loop representation of an open-loop Nash equilibrium are coupled, and are not symmetric. Therefore, the closed-loop representation of open-loop Nash equilibria is different from the outcome of closed-loop Nash equilibria, for Problem (MF-SDG), in general.

\section{Mean-Field LQ ZERO-Sum Stochastic DifFerential Games}

In this section, we will look at the situation for mean-field LQ zero-sum stochastic differential games. According to (1.6), let us simplify some notation:

$$
\left\{\begin{array}{l}
Q_{1}=-Q_{2} \equiv Q, \quad \bar{Q}_{1}=-\bar{Q}_{2} \equiv \bar{Q}, \quad q_{1}(\cdot)=-q_{2}(\cdot) \equiv q(\cdot), \\
S_{1} \equiv\left(\begin{array}{l}
S_{11} \\
S_{12}
\end{array}\right)=-\left(\begin{array}{c}
S_{21} \\
S_{22}
\end{array}\right) \equiv-S_{2} \equiv\left(\begin{array}{c}
S^{1} \\
S^{2}
\end{array}\right) \equiv S, \quad \bar{S}_{1} \equiv\left(\begin{array}{c}
\bar{S}_{11} \\
\bar{S}_{12}
\end{array}\right)=-\left(\begin{array}{c}
\bar{S}_{21} \\
\bar{S}_{22}
\end{array}\right) \equiv-\bar{S}_{2} \equiv\left(\begin{array}{ll}
\bar{S}^{1} \\
\bar{S}^{2}
\end{array}\right) \equiv \bar{S} \\
R_{1} \equiv\left(\begin{array}{ll}
R_{111} & R_{112} \\
R_{121} & R_{122}
\end{array}\right)=-\left(\begin{array}{ll}
R_{211} & R_{212} \\
R_{221} & R_{222}
\end{array}\right) \equiv-R_{2} \equiv R \equiv\left(\begin{array}{ll}
R_{11} & R_{12} \\
R_{21} & R_{22}
\end{array}\right) \\
\bar{R}_{1} \equiv\left(\begin{array}{ll}
\bar{R}_{111} & \bar{R}_{112} \\
\bar{R}_{121} & \bar{R}_{122}
\end{array}\right)=-\left(\begin{array}{ll}
\bar{R}_{211} & \bar{R}_{212} \\
\bar{R}_{221} & \bar{R}_{222}
\end{array}\right) \equiv-\bar{R}_{2} \equiv \bar{R} \equiv\left(\begin{array}{ll}
\bar{R}_{11} & \bar{R}_{12} \\
\bar{R}_{21} & \bar{R}_{22}
\end{array}\right) \\
\rho_{1}(\cdot) \equiv\left(\begin{array}{c}
\rho_{11}(\cdot) \\
\rho_{12}(\cdot)
\end{array}\right)=-\left(\begin{array}{c}
\rho_{21}(\cdot) \\
\rho_{22}(\cdot)
\end{array}\right) \equiv-\rho_{2}(\cdot) \equiv\left(\begin{array}{c}
\rho^{1}(\cdot) \\
\rho^{2}(\cdot)
\end{array}\right) \equiv \rho(\cdot) .
\end{array}\right.
$$

Then the cost functional will be the following:

$$
\begin{gathered}
J_{1}\left(x ; u_{1}(\cdot), u_{2}(\cdot)\right)=-J_{2}\left(x ; u_{1}(\cdot), u_{2}(\cdot)\right) \equiv J\left(x ; u_{1}(\cdot), u_{2}(\cdot)\right) \equiv J(x ; u(\cdot)) \\
\equiv \mathbb{E} \int_{0}^{\infty}\left[\left\langle\left(\begin{array}{cc}
Q & S^{\top} \\
S & R
\end{array}\right)\left(\begin{array}{c}
X(t) \\
u(t)
\end{array}\right),\left(\begin{array}{c}
X(t) \\
u(t)
\end{array}\right)\right\rangle+2\left\langle\left(\begin{array}{c}
q(t) \\
\rho(t)
\end{array}\right),\left(\begin{array}{c}
X(t) \\
u(t)
\end{array}\right)\right\rangle\right. \\
+\left\langle\left(\begin{array}{cc}
\bar{Q} & \overline{S^{\top}} \\
\bar{S} & \bar{R}
\end{array}\right)\left(\begin{array}{c}
\mathbb{E}[X(t)] \\
\mathbb{E}[u(t)]
\end{array}\right),\left(\begin{array}{c}
\mathbb{E}[X(t)] \\
\mathbb{E}[u(t)]
\end{array}\right)\right\rangle \mathrm{d} t .
\end{gathered}
$$

Similar to Problem (MF-SDG), we assume that (H2) holds. Then, for any $x \in \mathbb{R}^{n}$, we define $\mathscr{U}_{a d}(x)$ and $\mathscr{U}_{\text {ad }}^{i}(x)$ $(i=1,2)$ similar to $(3.4)-(3.6)$. Let us state the following zero-sum problem.

Problem (MF-SDG) $)_{0}$. For any initial state $x \in \mathbb{R}^{n}$, Player 1 wants to find a control $u_{1}^{*}(\cdot)$ to minimize the cost functional $J\left(x ; u_{1}(\cdot), u_{2}(\cdot)\right)$, and Player 2 wants to find a control $u_{2}^{*}(\cdot)$ to maximize $J\left(x ; u_{1}(\cdot), u_{2}(\cdot)\right)$ respectively, subject to $(1.1)$ (or equivalently, $(3.2)$ ) such that $\left(u_{1}^{*}(\cdot), u_{2}^{*}(\cdot)\right) \in \mathcal{U}_{a d}(x)$.

Next, we introduce the following definitions.

Definition 4.1. (i) A pair $\left(u_{1}^{*}(\cdot), u_{2}^{*}(\cdot)\right) \in \mathcal{U}_{a d}(x)$ is called an open-loop saddle point of Problem (MF-SDG $)_{0}$ for the initial state $x \in \mathbb{R}^{n}$ if

$$
J\left(x ; u_{1}^{*}(\cdot), u_{2}(\cdot)\right) \leqslant J\left(x ; u_{1}^{*}(\cdot), u_{2}^{*}(\cdot)\right) \leqslant J\left(x ; u_{1}(\cdot), u_{2}^{*}(\cdot)\right), \quad \forall\left(u_{1}(\cdot), u_{2}(\cdot)\right) \in \mathscr{U}_{a d}(x) .
$$

(ii) A closed-strategy $\left(\boldsymbol{\Theta}^{*}, v^{*}(\cdot)\right) \in \mathscr{S}[A, \bar{A}, C, \bar{C} ; B, \bar{B}, D, \bar{D}] \times L_{\mathbb{F}}^{2}\left(\mathbb{R}^{m}\right)$ is called a closed-loop saddle point of Problem (MF-SDG) $)_{0}$ if for any $x \in \mathbb{R}^{n}, \boldsymbol{\Theta}_{1} \in \mathscr{S}^{1}\left(\boldsymbol{\Theta}_{2}^{*}\right), \boldsymbol{\Theta}_{2} \in \mathscr{S}^{2}\left(\boldsymbol{\Theta}_{1}^{*}\right), v_{1}(\cdot) \in L_{\mathbb{F}}^{2}\left(\mathbb{R}^{m_{1}}\right)$ and $v_{2}(\cdot) \in L_{\mathbb{F}}^{2}\left(\mathbb{R}^{m_{2}}\right)$,

$$
J\left(x ; \boldsymbol{\Theta}_{1}^{*}, v_{1}^{*}(\cdot) ; \boldsymbol{\Theta}_{2}, v_{2}(\cdot)\right) \leqslant J\left(x ; \boldsymbol{\Theta}^{*}, v^{*}(\cdot)\right) \leqslant J\left(x ; \boldsymbol{\Theta}_{1}, v_{1}(\cdot) ; \boldsymbol{\Theta}_{2}^{*}, v_{2}^{*}(\cdot)\right) .
$$

Similar as in Section 3, it is easy to see that $\left(\boldsymbol{\Theta}_{1}^{*}, v_{1}^{*}(\cdot) ; \boldsymbol{\Theta}_{2}^{*}, v_{2}^{*}(\cdot)\right)$ is a closed-loop saddle point of Problem $(\mathrm{MF}-\mathrm{SDG})_{0}$ if and only if one of the following holds: 
(i) For any $v_{1}(\cdot) \in L_{\mathbb{F}}^{2}\left(\mathbb{R}^{m_{1}}\right)$ and $v_{2}(\cdot) \in L_{\mathbb{F}}^{2}\left(\mathbb{R}^{m_{2}}\right)$,

$$
J\left(x ; \boldsymbol{\Theta}_{1}^{*}, v_{1}^{*}(\cdot) ; \boldsymbol{\Theta}_{2}^{*}, v_{2}(\cdot)\right) \leqslant J\left(x ; \boldsymbol{\Theta}^{*}, v^{*}(\cdot)\right) \leqslant J\left(x ; \boldsymbol{\Theta}_{1}^{*}, v_{1}(\cdot) ; \boldsymbol{\Theta}_{2}^{*}, v_{2}^{*}(\cdot)\right) .
$$

(ii) For any $u_{1}(\cdot) \in L_{\mathbb{F}}^{2}\left(\mathbb{R}^{m_{1}}\right)$ and $u_{2}(\cdot) \in L_{\mathbb{F}}^{2}\left(\mathbb{R}^{m_{2}}\right)$,

$$
J\left(x ; \boldsymbol{\Theta}_{1}^{*}, v_{1}^{*}(\cdot) ; u_{2}(\cdot)\right) \leqslant J\left(x ; \mathbf{\Theta}^{*}, v^{*}(\cdot)\right) \leqslant J\left(x ; u_{1}(\cdot) ; \mathbf{\Theta}_{2}^{*}, v_{2}^{*}(\cdot)\right) .
$$

Definition 4.2. The following maps

$$
\left\{\begin{array}{l}
V^{+}(x) \triangleq \inf _{u_{1}(\cdot) \in \mathscr{\mathscr { U }}_{a d}^{1}(x)} \sup _{u_{2}(\cdot) \in \mathscr{U}_{a d}^{2}(x)} J\left(x ; u_{1}(\cdot), u_{2}(\cdot)\right), \\
V^{-}(x) \triangleq \sup _{u_{2}(\cdot) \in \mathscr{\mathscr { U }}_{a d}^{2}(x)} \inf _{u_{1}(\cdot) \in \mathscr{U}_{a d}^{1}(x)} J\left(x ; u_{1}(\cdot), u_{2}(\cdot)\right),
\end{array}\right.
$$

are called the upper value function and the lower value function of Problem (MF-SDG) $)_{0}$, respectively. In the case that

$$
V^{+}(x)=V^{-}(x) \equiv V(x),
$$

we call the map $x \mapsto V(x)$ the value function of Problem (MF-SDG) $)_{0}$.

Now, let $\left(\boldsymbol{\Theta}^{*}, v^{*}(\cdot)\right) \in \mathbb{R}^{2 m \times n} \times L_{\mathbb{F}}^{2}\left(\mathbb{R}^{m}\right)$, and assume the open-loop saddle points of Problem (MF-SDG) 0 admit the closed-loop representation. Then by $(4.1)$, we see that $\left(P_{1}, \widehat{P}_{1}\right)=\left(-P_{2},-\widehat{P}_{2}\right) \equiv(P, \widehat{P})$ satisfy the same equations (see (3.33))

$$
\left\{\begin{array}{l}
P A+A^{\top} P+C^{\top} P C+Q+\left(P B+C^{\top} P D+S^{\top}\right) \Theta^{*}=0 \\
\widehat{P} \widehat{A}+\widehat{A}^{\top} \widehat{P}+\widehat{C}^{\top} P \widehat{C}+\widehat{Q}+\left(\widehat{P} \widehat{B}+\widehat{C}^{\top} P \widehat{D}+\widehat{S}^{\top}\right) \bar{\Theta}^{*}=0
\end{array}\right.
$$

and (3.34) is equivalent to

$$
\begin{cases}\Sigma_{o} \Theta^{*}+B^{\top} P+D^{\top} P C+S=0, & \Sigma_{o} \equiv R+D^{\top} P D \\ \bar{\Sigma}_{o} \bar{\Theta}^{*}+\widehat{B}^{\top} \widehat{P}+\widehat{D}^{\top} P \widehat{C}+\widehat{S}=0, & \bar{\Sigma}_{o} \equiv \widehat{R}+\widehat{D}^{\top} P \widehat{D}\end{cases}
$$

These equations (for $\Theta^{*}$ and $\bar{\Theta}^{*}$ ) are solvable if and only if

$$
\begin{cases}\mathscr{R}\left(B^{\top} P+D^{\top} P C+S\right) \subseteq \mathscr{R}\left(\Sigma_{o}\right), & \Theta^{*}=-\Sigma_{o}^{\dagger}\left(B^{\top} P+D^{\top} P C+S\right)+\left(I-\Sigma_{o}^{\dagger} \Sigma_{o}\right) \theta \\ \mathscr{R}\left(\widehat{B}^{\top} \widehat{P}+\widehat{D}^{\top} P \widehat{C}+\widehat{S}\right) \subseteq \mathscr{R}\left(\bar{\Sigma}_{o}\right), & \bar{\Theta}^{*}=-\bar{\Sigma}_{o}^{\dagger}\left(\widehat{B}^{\top} \widehat{P}+\widehat{D}^{\top} P \widehat{C}+\widehat{S}\right)+\left(I-\bar{\Sigma}_{o}^{\dagger} \bar{\Sigma}_{o}\right) \bar{\theta}\end{cases}
$$

for some $\theta, \bar{\theta} \in \mathbb{R}^{m \times n}$ are chosen such that $\left(\Theta^{*}, \bar{\Theta}^{*}\right) \in \mathscr{S}[A, \bar{A}, C, \bar{C} ; B, \bar{B}, D, \bar{D}]$. Putting (4.9) into (4.7), it yields

$$
\left\{\begin{array}{l}
P A+A^{\top} P+C^{\top} P C+Q-\left(P B+C^{\top} P D+S^{\top}\right) \Sigma_{o}^{\dagger}\left(B^{\top} P+D^{\top} P C+S\right)=0 \\
\widehat{P} \widehat{A}+\widehat{A}^{\top} \widehat{P}+\widehat{C}^{\top} P \widehat{C}+\widehat{Q}-\left(\widehat{P} \widehat{B}+\widehat{C}^{\top} P \widehat{D}+\widehat{S}^{\top}\right) \bar{\Sigma}_{o}^{\dagger}\left(\widehat{B}^{\top} \widehat{P}+\widehat{D}^{\top} P \widehat{C}+\widehat{S}\right)=0
\end{array}\right.
$$

which is a system of coupled AREs, and both of them are symmetric. Thus, we have $P, \widehat{P} \in \mathbb{S}^{n}$. Next, by (4.1) again, from the componentwise form of $(3.29)$, it is easy to see that $\left(\eta_{1}(\cdot), \zeta_{1}(\cdot)\right)=\left(-\eta_{2}(\cdot),-\zeta_{2}(\cdot)\right)$ 
$\equiv\left(\eta_{o}(\cdot), \zeta_{o}(\cdot)\right) \in \mathscr{X}[0, \infty) \times L_{\mathbb{F}}^{2}\left(\mathbb{R}^{n}\right)$ satisfies

$$
\begin{aligned}
-\mathrm{d} \eta_{o}(t)= & \left\{A^{\top} \eta_{o}(t)-\left(P B+C^{\top} P D+S^{\top}\right) \Sigma_{o}^{\dagger}\left\{B^{\top} \eta_{o}(t)+D^{\top}\left[\zeta_{o}(t)+P \sigma(t)\right]+\rho(t)\right\}\right. \\
& \left.+C^{\top}\left[\zeta_{o}(t)+P \sigma(t)\right]+P b(t)+q(t)\right\} \mathrm{d} t-\zeta_{o}(t) \mathrm{d} W(t), \quad t \geqslant 0
\end{aligned}
$$

with constraint

$$
B^{\top}\left(\eta_{o}-\mathbb{E}\left[\eta_{o}\right]\right)+D^{\top}\left(\zeta_{o}-\mathbb{E}\left[\zeta_{o}\right]\right)+D^{\top} P(\sigma-\mathbb{E}[\sigma])+\rho-\mathbb{E}[\rho] \in \mathcal{R}\left(\Sigma_{o}\right), \quad \text { a.e., a.s. }
$$

Likewise, by (4.1), from the componentwise form of $(3.30)$, we see that $\bar{\eta}_{1}(\cdot)=-\bar{\eta}_{2}(\cdot) \equiv \bar{\eta}_{o}(\cdot) \in L^{2}\left(\mathbb{R}^{n}\right)$ satisfy the following:

$$
\begin{aligned}
\dot{\bar{\eta}}_{o}(t) & +\widehat{A}^{\top} \bar{\eta}_{o}(t)-\left(\widehat{P} \widehat{B}+\widehat{C}^{\top} P \widehat{D}+\widehat{S}^{\top}\right) \bar{\Sigma}_{o}^{\dagger}\left(\widehat{B}^{\top} \bar{\eta}_{o}(t)+\mathbb{E}\left[\bar{\zeta}_{o}(t)\right]+P \mathbb{E}[\sigma(t)]+\mathbb{E}[\rho(t)]\right) \\
& +\widehat{C}^{\top}\left(\mathbb{E}\left[\bar{\zeta}_{o}(t)\right]+P \mathbb{E}[\sigma(t)]\right)+\mathbb{E}[q(t)]+\widehat{P} \mathbb{E}[b(t)]=0, \quad t \geqslant 0,
\end{aligned}
$$

with constraint

$$
\widehat{B}^{\top} \bar{\eta}_{o}+\widehat{D}^{\top} \mathbb{E}\left[\zeta_{o}\right]+\widehat{D}^{\top} P \mathbb{E}[\sigma]+\mathbb{E}[\rho] \in \mathscr{R}\left(\bar{\Sigma}_{o}\right), \quad \text { a.e. }
$$

Finally, from (3.9), it is direct that the closed-loop representation of an open-loop saddle point is given by

$$
\begin{aligned}
u^{*}(\cdot)=\{ & \left.-\Sigma_{o}^{\dagger}\left(B^{\top} P+D^{\top} P C+S\right)+\left(I-\Sigma_{o}^{\dagger} \Sigma_{o}\right) \theta\right\}\left(X^{*}(\cdot)-\mathbb{E}\left[X^{*}(\cdot)\right]\right) \\
& +\left\{-\bar{\Sigma}_{o}^{\dagger}\left(\widehat{B}^{\top} \widehat{P}+\widehat{D}^{\top} P \widehat{C}+\widehat{S}\right)+\left(I-\bar{\Sigma}_{o}^{\dagger} \overline{\Sigma_{o}}\right) \bar{\theta}\right\} \mathbb{E}\left[X^{*}(\cdot)\right] \\
& -\Sigma_{o}^{\dagger}\left\{B^{\top}\left(\eta_{o}(\cdot)-\mathbb{E}\left[\eta_{o}(\cdot)\right]\right)+D^{\top}\left[\zeta_{o}(\cdot)-\mathbb{E}\left[\zeta_{o}(\cdot)\right]+P(\sigma(\cdot)-\mathbb{E}[\sigma(\cdot)])\right]+\rho(\cdot)-\mathbb{E}[\rho(\cdot)]\right\} \\
& -\bar{\Sigma}_{o}^{\dagger}\left\{\widehat{B}^{\top} \bar{\eta}_{o}(\cdot)+\widehat{D}^{\top}\left(P \mathbb{E}[\sigma(\cdot)]+\mathbb{E}\left[\zeta_{o}(\cdot)\right]\right)+\mathbb{E}[\rho(\cdot)]+\bar{\rho}(\cdot)\right\} \\
& +\left(I-\Sigma_{o}^{\dagger} \Sigma_{o}\right)(\nu(\cdot)-\mathbb{E}[\nu(\cdot)])+\left(I-\bar{\Sigma}_{o}^{\dagger} \bar{\Sigma}_{o}\right) \bar{\nu}(\cdot),
\end{aligned}
$$

where $\nu(\cdot), \bar{\nu}(\cdot) \in L^{2}\left(\mathbb{R}^{m}\right)$, and $X^{*}(\cdot) \in \mathscr{X}[0, \infty)$ is the solution to (3.8) corresponding to $u^{*}(\cdot)$.

To summarize, we have the following result.

Theorem 4.3. Let (H2) hold and the initial state $x \in \mathbb{R}^{n}$ be given. Then any open-loop saddle point $u^{*}(\cdot)$ of Problem (MF-SDG) admits a closed-loop representation if and only if the following hold:

(i) The following convexity-concavity condition holds: For $i=1,2$,

$$
\begin{gathered}
(-1)^{i-1} \mathbb{E} \int_{0}^{\infty}\left[\left\langle Q X_{i}(t), X_{i}(t)\right\rangle+2\left\langle S^{i} X_{i}(t), u_{i}(t)\right\rangle+\left\langle R_{i i} u_{i}(t), u_{i}(t)\right\rangle+\left\langle\bar{Q} \mathbb{E}\left[X_{i}(t)\right], \mathbb{E}\left[X_{i}(t)\right]\right\rangle\right. \\
\left.+2\left\langle\bar{S}^{i} \mathbb{E}\left[X_{i}(t)\right], \mathbb{E}\left[u_{i}(t)\right]\right\rangle+\left\langle\bar{R}_{i i} \mathbb{E}\left[u_{i}(t)\right], \mathbb{E}\left[u_{i}(t)\right]\right\rangle\right] \mathrm{d} t \geqslant 0, \forall u(\cdot) \equiv\left(\begin{array}{l}
u_{1}(\cdot) \\
u_{2}(\cdot)
\end{array}\right) \in \mathscr{U}_{a d}(x),
\end{gathered}
$$

where $X_{i}(\cdot) \in \mathscr{X}[0, \infty)$ is the solution to MF-SDE (3.20).

(ii) System (4.10) admits a static stabilizing solution $(P, \widehat{P}) \in \mathbb{S}^{n} \times \mathbb{S}^{n}$, such that the solution $\left(\eta_{o}(\cdot), \zeta_{o}(\cdot)\right) \in$ $\mathscr{X}[0, \infty) \times L_{\mathbb{F}}^{2}\left(\mathbb{R}^{n}\right)$ to (4.11) satisfies (4.12), and the solution $\bar{\eta}_{o}(\cdot) \in L^{2}\left(\mathbb{R}^{n}\right)$ to (4.13) satisfies (4.14).

In the above case, any open-loop saddle point admits the closed-loop representation (4.15).

The following result characterizes the closed-loop saddle points of Problem (MF-SDG) ${ }_{0}$.

Theorem 4.4. Problem $(M F-S D G)_{0}$ admits a closed-loop saddle point $\left(\Theta^{*}, \bar{\Theta}^{*}, v^{*}(\cdot)\right)$ if and only if the following statements hold: 
(i) The following system:

$$
\left\{\begin{array}{l}
P_{c} A+A^{\top} P_{c}+C^{\top} P_{c} C+Q-\left(P_{c} B+C^{\top} P_{c} D+S^{\top}\right) \Sigma_{c}^{\dagger}\left(B^{\top} P_{c}+D^{\top} P_{c} C+S\right)=0 \\
\widehat{P}_{c} \widehat{A}+\widehat{A}^{\top} \widehat{P}_{c}+\widehat{C}^{\top} P_{c} \widehat{C}+\widehat{Q}-\left(\widehat{P}_{c} \widehat{B}+\widehat{C}^{\top} P_{c} \widehat{D}+\widehat{S}^{\top}\right) \bar{\Sigma}_{c}^{\dagger}\left(\widehat{B}^{\top} \widehat{P}_{c}+\widehat{D}^{\top} P_{c} \widehat{C}+\widehat{S}\right)=0 \\
\mathscr{R}\left(B^{\top} P_{c}+D^{\top} P_{c} C+S\right) \subseteq \mathscr{R}\left(\Sigma_{c}\right), \quad \mathscr{R}\left(\widehat{B}^{\top} \widehat{P}_{c}+\widehat{D}^{\top} P_{c} \widehat{C}+\widehat{S}\right) \subseteq \mathscr{R}\left(\bar{\Sigma}_{c}\right)
\end{array}\right.
$$

admits a static stabilizing solution $\left(P_{c}, \widehat{P}_{c}\right) \in \mathbb{S}^{n} \times \mathbb{S}^{n}$ such that

$$
\begin{cases}R_{11}+D_{1}^{\top} P_{c} D_{1} \geqslant 0, & \widehat{R}_{11}+\widehat{D}_{1}^{\top} P_{c} \widehat{D}_{1} \geqslant 0 \\ R_{22}+D_{2}^{\top} P_{c} D_{2} \leqslant 0, & \widehat{R}_{22}+\widehat{D}_{2}^{\top} P_{c} \widehat{D}_{2} \leqslant 0\end{cases}
$$

(ii) The following BSDE on $[0, \infty)$ :

$$
\left\{\begin{array}{r}
-\mathrm{d} \eta_{c}(t)=\left\{A^{\top} \eta_{c}(t)-\left(P_{c} B+C^{\top} P_{c} D+S^{\top}\right) \Sigma_{c}^{\dagger}\left[B^{\top} \eta_{c}(t)+D^{\top}\left(\zeta_{c}(t)+P_{c} \sigma(t)\right)+\rho(t)\right]\right. \\
\left.+C^{\top}\left[\zeta_{c}(t)+P_{c} \sigma(t)\right]+P_{c} b(t)+q(t)\right\} \mathrm{d} t-\zeta_{c}(t) \mathrm{d} W(t), \quad t \geqslant 0, \\
B^{\top}\left[\eta_{c}(t)-\mathbb{E}\left[\eta_{c}(t)\right]+D^{\top}\left[\zeta_{c}(t)-\mathbb{E}\left[\zeta_{c}(t)\right]\right]+D^{\top} P_{c}[\sigma(t)-\mathbb{E}[\sigma(t)]]+\rho(t)-\mathbb{E}[\rho(t)] \in \mathscr{R}\left(\Sigma_{c}\right),\right. \\
\text { a.e. } t \in[0, \infty), \text { a.s. },
\end{array}\right.
$$

admits a solution $\left(\eta_{c}(\cdot), \zeta_{c}(\cdot)\right) \in \mathscr{X}[0, \infty) \times L_{\mathbb{F}}^{2}\left(\mathbb{R}^{n}\right)$, and the following ODE:

$$
\left\{\begin{array}{c}
\dot{\bar{\eta}}_{c}(t)+\widehat{A}^{\top} \bar{\eta}_{c}(t)-\left(\widehat{P} \widehat{B}+\widehat{C}^{\top} P_{c} \widehat{D}+\widehat{S}^{\top}\right) \bar{\Sigma}_{c}^{\dagger}\left\{\widehat{B}^{\top} \bar{\eta}_{c}(t)+\widehat{D}^{\top}\left(\mathbb{E}\left[\bar{\zeta}_{o}(t)\right]+P_{c} \mathbb{E}[\sigma(t)]\right)+\mathbb{E}[\bar{\rho}(t)]\right\} \\
+\widehat{C}^{\top}\left(\mathbb{E}\left[\bar{\zeta}_{o}(t)\right]+P_{c} \mathbb{E}[\sigma(t)]\right)+\mathbb{E}[q(t)]+\widehat{P}_{c} \mathbb{E}[b(t)], \quad t \geqslant 0, \\
\widehat{B}^{\top} \bar{\eta}_{c}(t)+\widehat{D}^{\top} \mathbb{E}\left[\zeta_{c}(t)\right]+\widehat{D}^{\top} P_{c} \mathbb{E}[\sigma(t)]+\mathbb{E}[\rho(t)] \in \mathscr{R}\left(\bar{\Sigma}_{c}\right), \quad \text { a.e. } t \in[0, \infty),
\end{array}\right.
$$

admits a solution $\bar{\eta}_{c}(\cdot) \in L^{2}\left(\mathbb{R}^{n}\right)$. In the above case, the closed-loop saddle point is given by

$$
\left\{\begin{array}{l}
\Theta^{*}=-\Sigma_{c}^{\dagger}\left(B^{\top} P_{c}+D^{\top} P_{c} C+S\right)+\left(I-\Sigma_{c}^{\dagger} \Sigma_{c}\right) \theta \\
\bar{\Theta}^{*}=-\bar{\Sigma}_{c}^{\dagger}\left[\widehat{B}^{\top} \Pi_{c}+\widehat{D}^{\top} P_{c} \widehat{C}+\widehat{S}\right]+\left(I-\bar{\Sigma}_{c}^{\dagger} \bar{\Sigma}_{c}\right) \bar{\theta}
\end{array}\right.
$$

where $\theta, \bar{\theta} \in \mathbb{R}^{m \times n}$ are chosen such that $\left(\Theta^{*}, \bar{\Theta}^{*}\right) \in \mathscr{S}[A, \bar{A}, C, \bar{C} ; B, \bar{B}, D, \bar{D}]$, and

$$
\begin{aligned}
v^{*}(\cdot)= & -\Sigma_{c}^{\dagger}\left\{B^{\top}\left(\eta_{c}(\cdot)-\mathbb{E}\left[\eta_{c}(\cdot)\right]\right)+D^{\top}\left(\zeta_{c}(\cdot)-\mathbb{E}\left[\zeta_{c}(\cdot)\right]\right)+D^{\top} P(\sigma(\cdot)-\mathbb{E}[\sigma(\cdot)])+\rho(\cdot)-\mathbb{E}[\rho(\cdot)]\right\} \\
& -\bar{\Sigma}_{c}^{\dagger}\left\{\widehat{B}^{\top} \bar{\eta}_{c}(\cdot)+\widehat{D}^{\top}\left(P_{c} \mathbb{E}[\sigma(\cdot)]+\mathbb{E}\left[\zeta_{c}(\cdot)\right]\right)+\mathbb{E}[\rho(\cdot)]\right\} \\
& +\left(I-\Sigma_{c}^{\dagger} \Sigma_{c}\right)(\nu(\cdot)-\mathbb{E}[\nu(\cdot)])+\left(I-\bar{\Sigma}_{c}^{\dagger} \bar{\Sigma}_{c}\right) \bar{\nu}(\cdot)
\end{aligned}
$$

for some $\nu(\cdot) \in L_{\mathbb{F}}^{2}\left(\mathbb{R}^{m}\right), \bar{\nu}(\cdot) \in L^{2}\left(\mathbb{R}^{m}\right)$.

Proof. Let $\left(\boldsymbol{\Theta}^{*}, v^{*}(\cdot)\right)$ be a closed-loop saddle point of Problem (MF-SDG) $)_{0}$. System (3.35) for $\left(P_{1}, \widehat{P}_{1}\right) \equiv$ $\left(-P_{2},-\widehat{P}_{2}\right)=\left(P_{c}, \widehat{P}_{c}\right)$ becomes

$$
\left\{\begin{array}{l}
P_{c} A+A^{\top} P_{c}+C^{\top} P_{c} C+Q+\left(\Theta^{*}\right)^{\top}\left(R+D^{\top} P_{c} D\right) \Theta^{*} \\
\quad+\left(P_{c} B+C^{\top} P_{c} D+S^{\top}\right) \Theta^{*}+\left(\Theta^{*}\right)^{\top}\left(B^{\top} P_{c}+D^{\top} P_{c} C+S\right)=0 \\
\widehat{P}_{c} \widehat{A}+\widehat{A}^{\top} \widehat{P}_{c}+\widehat{C}^{\top} P_{c} \widehat{C}+\widehat{Q}+\left(\bar{\Theta}^{*}\right)^{\top}\left(\widehat{R}+\widehat{D}^{\top} P_{c} \widehat{D}\right) \bar{\Theta}^{*} \\
\quad+\left(\widehat{P}_{c} \widehat{B}+\widehat{C}^{\top} P_{c} \widehat{D}+\widehat{S}^{\top}\right) \bar{\Theta}^{*}+\left(\bar{\Theta}^{*}\right)^{\top}\left(\widehat{B}^{\top} \widehat{P}_{c}+\widehat{D}^{\top} P_{c} \widehat{C}+\widehat{S}\right)=0
\end{array}\right.
$$


Thus, (3.36) becomes

$$
\left\{\begin{array}{l}
B^{\top} P_{c}+D^{\top} P_{c} C+S+\Sigma_{c} \Theta^{*}=0 \\
\widehat{B}^{\top} \widehat{P}_{c}+\widehat{D}^{\top} P_{c} \widehat{C}+\widehat{S}+\bar{\Sigma}_{c} \bar{\Theta}^{*}=0
\end{array}\right.
$$

This system is solvable if and only if

$$
\begin{cases}\mathscr{R}\left(B^{\top} P_{c}+D^{\top} P_{c} C+S\right) \subseteq \mathscr{R}\left(\Sigma_{c}\right), & \Theta^{*}=-\Sigma_{c}^{\dagger}\left(B^{\top} P_{c}+D^{\top} P_{c} C+S\right)+\left(I-\Sigma_{c}^{\dagger} \Sigma_{c}\right) \theta \\ \mathscr{R}\left(\widehat{B}^{\top} \widehat{P}_{c}+\widehat{D}^{\top} P_{c} \widehat{C}+\widehat{S}\right) \subseteq \mathscr{R}\left(\bar{\Sigma}_{c}\right), & \bar{\Theta}^{*}=-\bar{\Sigma}_{c}^{\dagger}\left(\widehat{B}^{\top} \widehat{P}_{c}+\widehat{D}^{\top} P_{c} \widehat{C}+\widehat{S}\right)+\left(I-\bar{\Sigma}_{c}^{\dagger} \bar{\Sigma}_{c}\right) \bar{\theta}\end{cases}
$$

where $\theta, \bar{\theta} \in \mathbb{R}^{m \times n}$ are chosen such that $\left(\Theta^{*}, \bar{\Theta}^{*}\right) \in \mathscr{S}[A, \bar{A}, C, \bar{C} ; B, \bar{B}, D, \bar{D}]$. This proves (4.21). Putting (4.24) into (4.23) yields that (4.17) admits a static stabilizing solution $\left(P_{c}, \widehat{P}_{c}\right) \in \mathbb{S}^{n} \times \mathbb{S}^{n}$. (4.18) can be easily obtained from (3.37) and (4.1). (4.19) and (4.20) can be similarly proved from (ii) of Theorem 3.7.

Finally, from (3.44) we have

$$
\begin{gathered}
v^{*}(\cdot)-\mathbb{E}\left[v^{*}(\cdot)\right]=-\Sigma_{c}^{\dagger}\left\{B^{\top}\left(\eta_{c}(\cdot)-\mathbb{E}\left[\eta_{c}(\cdot)\right]\right)+D^{\top}\left(\zeta_{c}(\cdot)-\mathbb{E}\left[\zeta_{c}(\cdot)\right]\right)+D^{\top} P_{c}(\sigma(\cdot)-\mathbb{E}[\sigma(\cdot)])\right. \\
+\rho(\cdot)-\mathbb{E}[\rho(\cdot)]\}+\left(I-\Sigma_{c}^{\dagger} \Sigma_{c}\right)(\nu(\cdot)-\mathbb{E}[\nu(\cdot)]),
\end{gathered}
$$

for some $\nu(\cdot) \in L_{\mathbb{F}}^{2}\left(\mathbb{R}^{m}\right)$. It follows from (3.46) that we get

$$
\mathbb{E}\left[v^{*}(\cdot)\right]=-\bar{\Sigma}_{c}^{\dagger}\left\{\widehat{B}^{\top} \bar{\eta}_{c}(\cdot)+\widehat{D}^{\top}\left(P_{c} \mathbb{E}[\sigma(\cdot)]+\mathbb{E}\left[\zeta_{c}(\cdot)\right]\right)+\mathbb{E}[\rho(\cdot)]\right\}+\left(I-\bar{\Sigma}_{c}^{\dagger} \bar{\Sigma}_{c}\right) \bar{\nu}(\cdot),
$$

for some $\bar{\nu}(\cdot) \in L^{2}\left(\mathbb{R}^{m}\right)$. Combining the above two expressions leads to (4.22). The proof is complete.

Comparing Theorems 4.3 and 4.4, we have the following result which might not be true for general non-zero sum differential games (see [47]).

Theorem 4.5. If both the closed-loop representation of open-loop saddle points and the closed-loop saddle points of Problem (MF-SDG) $)_{0}$ exist, then the closed-loop representation coincides with the outcome of the closed-loop saddle points. In the above case, the value function admits the following representation:

$$
\begin{aligned}
V(x) & =\langle\widehat{P} x, x\rangle+2\left\langle\bar{\eta}_{c}(0), x\right\rangle \\
& +\mathbb{E} \int_{0}^{\infty}\left[\langle P \sigma(t), \sigma(t)\rangle+2\left\langle\eta_{c}(t), b(t)-\mathbb{E}[b(t)]\right\rangle+2\left\langle\zeta_{c}(t), \sigma(t)\right\rangle+2\left\langle\bar{\eta}_{c}(t), \mathbb{E}[b(t)]\right\rangle\right. \\
& -\left|\left(\Sigma^{\dagger}\right)^{\frac{1}{2}}\left\{B^{\top}\left(\eta_{c}(t)-\mathbb{E}\left[\eta_{c}(t)\right]\right)+D^{\top}\left[\zeta_{c}(t)-\mathbb{E}\left[\zeta_{c}(t)\right]+P(\sigma(t)-\mathbb{E}[\sigma(t)])\right]+\rho(t)-\mathbb{E}[\rho(t)]\right\}\right|^{2} \\
& \left.-\left|\left(\bar{\Sigma}^{\dagger}\right)^{\frac{1}{2}}\left\{\widehat{B}^{\top} \bar{\eta}_{c}(t)+\widehat{D}^{\top}\left(\mathbb{E}\left[\zeta_{c}(t)\right]+P \mathbb{E}[\sigma(t)]\right)+\mathbb{E}[\rho(t)]\right\}\right|^{2}\right] \mathrm{d} t,
\end{aligned}
$$

where $\Sigma \equiv \Sigma_{o} \equiv \Sigma_{c} \triangleq R+D^{\top} P D \equiv R+D^{\top} P_{c} D, \bar{\Sigma} \equiv \bar{\Sigma}_{o} \equiv \bar{\Sigma}_{c} \triangleq \widehat{R}+\widehat{D}^{\top} P \widehat{D} \equiv \widehat{R}+\widehat{D}^{\top} P_{c} \widehat{D}, \widehat{P} \equiv \widehat{P}_{c}$.

Proof. Let $\left(\Theta^{*}, v^{*}(\cdot)\right)$ be a closed-loop saddle point of Problem (MF-SDG) $)_{0}$. By (4.6), the outcome

$$
u^{*}(\cdot)=\Theta^{*}\left(X^{*}(\cdot)-\mathbb{E}\left[X^{*}(\cdot)\right]\right)+\bar{\Theta}^{*} \mathbb{E}\left[X^{*}(\cdot)\right]+v^{*}(\cdot)
$$


of $\left(\Theta^{*}, \bar{\Theta}^{*}, v^{*}(\cdot)\right)$ is an open-loop saddle point of Problem $(\text { MF-SDG })_{0}$, where $X^{*}(\cdot) \in \mathscr{X}[0, \infty)$ is the solution to



Thus, similar to the proof of the sufficiency on Theorem 3.3, noting (4.25) and (4.26), we have

$$
\begin{aligned}
& J\left(x ; \Theta^{*}\left(X^{*}(\cdot)-\mathbb{E}\left[X^{*}(\cdot)\right]\right)+\bar{\Theta}^{*} \mathbb{E}\left[X^{*}(\cdot)\right]+v^{*}(\cdot)\right)-\left\langle\widehat{P}_{c} x+2 \bar{\eta}_{c}(0), x\right\rangle \\
& =\mathbb{E} \int_{0}^{\infty}\left[\left\langle\Sigma_{c}\left(v^{*}-\mathbb{E}\left[v^{*}\right]\right), v^{*}-\mathbb{E}\left[v^{*}\right]\right\rangle+2\left\langle B^{\top} \eta_{c}+D^{\top}\left(\zeta_{c}+P_{c} \sigma\right)+\rho, v^{*}-\mathbb{E}\left[v^{*}\right]\right\rangle\right. \\
& +\left\langle\bar{\Sigma}_{c} \mathbb{E}\left[v^{*}\right], \mathbb{E}\left[v^{*}\right]\right\rangle+2\left\langle\widehat{B}^{\top} \bar{\eta}_{c}+\widehat{D}^{\top}\left(\mathbb{E}\left[\zeta_{c}\right]+P_{c} \mathbb{E}[\sigma]\right)+\mathbb{E}[\rho], \mathbb{E}\left[v^{*}\right]\right\rangle \\
& \left.+\left\langle P_{c} \sigma, \sigma\right\rangle+2\left\langle\eta_{c}, b-\mathbb{E}[b]\right\rangle+2\left\langle\zeta_{c}, \sigma\right\rangle+2\left\langle\bar{\eta}_{c}, \mathbb{E}[b]\right\rangle\right] \mathrm{d} t \\
& =\mathbb{E} \int_{0}^{\infty}\left[\left\langle P_{c} \sigma, \sigma\right\rangle+2\left\langle\eta_{c}, b-\mathbb{E}[b]\right\rangle+2\left\langle\zeta_{c}, \sigma\right\rangle+2\left\langle\bar{\eta}_{c}, \mathbb{E}[b]\right\rangle\right. \\
& -\left|\left(\Sigma_{c}^{\dagger}\right)^{\frac{1}{2}}\left\{B^{\top}\left(\eta_{c}-\mathbb{E}\left[\eta_{c}\right]\right)+D^{\top}\left(\zeta_{c}-\mathbb{E}\left[\zeta_{c}\right]\right)+D^{\top} P_{c}(\sigma-\mathbb{E}[\sigma])+\rho-\mathbb{E}[\rho]\right\}\right|^{2} \\
& \left.-\left|\left(\bar{\Sigma}_{c}^{\dagger}\right)^{\frac{1}{2}}\left\{\widehat{B}^{\top} \bar{\eta}_{c}+\widehat{D}^{\top}\left(P_{c} \mathbb{E}[\sigma]+\mathbb{E}\left[\zeta_{c}\right]\right)+\mathbb{E}[\rho]\right\}\right|^{2}\right] \mathrm{d} t
\end{aligned}
$$

where $\left(P_{c}, \widehat{P}_{c}\right)$ satisfies $(4.17),\left(\eta_{c}(\cdot), \zeta_{c}(\cdot)\right)$ satisfies (4.19) and $\bar{\eta}_{c}(\cdot)$ satisfies (4.20). By Theorem 4.4, $\left(\Theta^{*}, \bar{\Theta}^{*}, v^{*}(\cdot)\right)$ is given by $(4.21)-(4.22)$.

On the other hand, let $(P, \widehat{P})$ be the solution pair to $(4.10),\left(\eta_{o}(\cdot), \zeta_{o}(\cdot)\right)$ be the solution to $(4.11), \bar{\eta}_{o}(\cdot)$ be the solution to (4.13), and choose $\theta, \bar{\theta} \in \mathbb{R}^{m \times n}, \nu(\cdot), \bar{\nu}(\cdot) \in L^{2}\left(\mathbb{R}^{m}\right)$, such that the following

$$
\left\{\begin{aligned}
\Theta^{* *}=- & \Sigma_{o}^{\dagger}\left(B^{\top} P+D^{\top} P C+S\right)+\left(I-\Sigma_{o}^{\dagger} \Sigma_{o}\right) \theta, \quad \bar{\Theta}^{* *}=-\bar{\Sigma}_{o}^{\dagger}\left(\widehat{B}^{\top} \widehat{P}+\widehat{D}^{\top} P \widehat{C}+\widehat{S}\right)+\left(I-\bar{\Sigma}_{o}^{\dagger} \bar{\Sigma}_{o}\right) \bar{\theta}, \\
v^{* *}(\cdot)=- & -\Sigma_{o}^{\dagger}\left\{B^{\top}\left(\eta_{o}(\cdot)-\mathbb{E}\left[\eta_{o}(\cdot)\right]\right)+D^{\top}\left(\zeta_{o}(\cdot)-\mathbb{E}\left[\zeta_{o}(\cdot)\right]\right)+D^{\top} P(\sigma(\cdot)-\mathbb{E}[\sigma(\cdot)])+\rho(\cdot)-\mathbb{E}[\rho(\cdot)]\right\} \\
& -\bar{\Sigma}_{o}^{\dagger}\left\{\widehat{B}^{\top} \bar{\eta}_{o}(\cdot)+\widehat{D}^{\top}\left(P \mathbb{E}[\sigma(\cdot)]+\mathbb{E}\left[\zeta_{o}(\cdot)\right]\right)+\mathbb{E}[\rho(\cdot)]\right\}+\left(I-\Sigma_{o}^{\dagger} \Sigma_{o}\right)(\nu(\cdot)-\mathbb{E}[\nu(\cdot)])+\left(I-\bar{\Sigma}_{o}^{\dagger} \bar{\Sigma}_{o}\right) \bar{\nu}(\cdot),
\end{aligned}\right.
$$

satisfies $\left(\Theta^{* *}, \bar{\Theta}^{* *}\right) \in \mathscr{S}[A, \bar{A}, C, \bar{C} ; B, \bar{B}, D, \bar{D}]$. For any initial state $x$, define $u^{* *}(\cdot) \in \mathscr{U}_{a d}(x)$ as follows:

$$
u^{* *}(\cdot)=\Theta^{* *}\left\{X^{* *}(\cdot)-\mathbb{E}\left[X^{* *}(\cdot)\right]\right\}+\bar{\Theta}^{* *} \mathbb{E}\left[X^{* *}(\cdot)\right]+v^{* *}(\cdot),
$$

where $X^{* *}(\cdot) \in \mathscr{X}[0, \infty)$ is the solution to

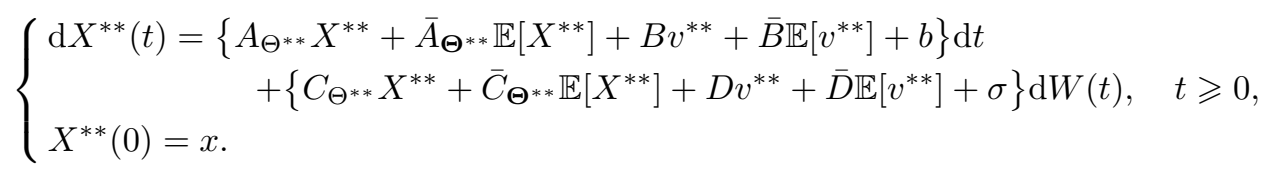


By Theorem 4.3, $u^{* *}(\cdot)$ is an open-loop saddle point of Problem (MF-SDG) $)_{0}$ for $x$. By the same argument as (4.28), noting (4.12) and (4.14), we obtain

$$
\begin{aligned}
& J\left(x ; \Theta^{* *}\left(X^{* *}(\cdot)-\mathbb{E}\left[X^{* *}(\cdot)\right]\right)+\bar{\Theta}^{* *} \mathbb{E}\left[X^{* *}(\cdot)\right]+v^{* *}(\cdot)\right)-\left\langle\widehat{P} x+2 \bar{\eta}_{o}(0), x\right\rangle \\
& =\mathbb{E} \int_{0}^{\infty}\left[\left\langle\Sigma_{o}\left(v^{* *}-\mathbb{E}\left[v^{* *}\right]\right), v^{* *}-\mathbb{E}\left[v^{* *}\right]\right\rangle+2\left\langle B^{\top} \eta_{o}+D^{\top} \zeta_{o}+D^{\top} P \sigma+\rho, v^{* *}-\mathbb{E}\left[v^{* *}\right]\right\rangle\right. \\
& \left.+\left\langle\bar{\Sigma}_{o} \mathbb{E}\left[v^{* *}\right], \mathbb{E}\left[v^{* *}\right]\right\rangle+2\left\langle\widehat{B}^{\top} \bar{\eta}_{o}+\widehat{D}^{\top} P \mathbb{E}[\sigma]+\mathbb{E}\left[\zeta_{o}\right]\right)+\mathbb{E}[\rho], \mathbb{E}\left[v^{* *}\right]\right\rangle \\
& \left.+\langle P \sigma, \sigma\rangle+2\left\langle\eta_{o}, b-\mathbb{E}[b]\right\rangle+2\left\langle\zeta_{o}, \sigma\right\rangle+2\left\langle\bar{\eta}_{o}, \mathbb{E}[b]\right\rangle\right] \mathrm{d} t \\
& =\mathbb{E} \int_{0}^{\infty}\left[\langle P \sigma, \sigma\rangle+2\left\langle\eta_{o}, b-\mathbb{E}[b]\right\rangle+2\left\langle\zeta_{o}, \sigma\right\rangle+2\left\langle\bar{\eta}_{o}, \mathbb{E}[b]\right\rangle\right. \\
& -\left|\left(\Sigma_{o}^{\dagger}\right)^{\frac{1}{2}}\left\{B^{\top}\left(\eta_{o}-\mathbb{E}\left[\eta_{o}\right]\right)+D^{\top}\left(\zeta_{o}-\mathbb{E}\left[\zeta_{o}\right]\right)+D^{\top} P(\sigma-\mathbb{E}[\sigma])+\rho-\mathbb{E}[\rho]\right\}\right|^{2} \\
& \left.-\left|\left(\bar{\Sigma}_{o}^{\dagger}\right)^{\frac{1}{2}}\left\{\widehat{B}^{\top} \bar{\eta}_{o}+\widehat{D}^{\top}\left(P \mathbb{E}[\sigma]+\mathbb{E}\left[\zeta_{o}\right]\right)+\mathbb{E}[\rho]\right\}\right|^{2}\right] \mathrm{d} t \text {. }
\end{aligned}
$$

Since both $u^{* *}(\cdot) \equiv\left(u_{1}^{* *}(\cdot), u_{2}^{* *}(\cdot)\right)$ and $u^{*}(\cdot) \equiv\left(u_{1}^{*}(\cdot), u_{2}^{*}(\cdot)\right)$ are open-loop saddle points of Problem (MF-SDG $)_{0}$ for $x$, we have

$$
J\left(x ; u_{1}^{*}(\cdot), u_{2}^{*}(\cdot)\right) \leqslant J\left(x ; u_{1}^{* *}(\cdot), u_{2}^{*}(\cdot)\right) \leqslant J\left(x ; u_{1}^{* *}(\cdot), u_{2}^{* *}(\cdot)\right) \leqslant J\left(x ; u_{1}^{*}(\cdot), u_{2}^{* *}(\cdot)\right) \leqslant J\left(x ; u_{1}^{*}(\cdot), u_{2}^{*}(\cdot)\right) .
$$

Therefore, $J\left(x ; u^{*}(\cdot)\right)=J\left(x ; u^{* *}(\cdot)\right)$ for all $x$, which, together with (4.28) and (4.29), yields

$$
P_{c}=P, \quad \widehat{P}_{c}=\widehat{P}, \quad \eta_{c}(\cdot)=\eta_{o}(\cdot), \quad \zeta_{c}(\cdot)=\zeta_{o}(\cdot), \quad \bar{\eta}_{c}(\cdot)=\bar{\eta}_{o}(\cdot)
$$

Thus, the value function is given by (4.27). The proof is complete.

Finally, we have the following corollary for Problem (MF-SLQ), since it is a special case of Problem (MFSDG) $)_{0}$ when $m_{2}=0$.

Corollary 4.6. For Problem (MF-SLQ), if the open-loop optimal control admits a closed-loop representation, then each open-loop optimal control must be an outcome of a closed-loop optimal strategy.

\section{EXAMPles}

In this section, we present several examples illustrating the results established in the previous sections. We mainly concentrate the closed-loop representation of the open-loop Nash equilibria/saddle points and the closed-loop Nash equilibria/saddle points.

The following example shows that for the mean-field LQ zero-sum stochastic differential game, it may happen that the closed-loop saddle point uniquely exists, which coincides with the closed-loop representation of the openloop saddle point. Moreover, the system of generalized AREs admits static stabilizing solutions and the system $[A, \bar{A}, C, \bar{C} ; B, \bar{B}, D, \bar{D}]$ is MF- $L^{2}$-stabilizable.

Example 5.1. Consider the following two-dimensional state equation

$$
\left\{\begin{array}{l}
\mathrm{d} X(t)=\left\{\begin{array}{l}
A X(t)+\bar{A} \mathbb{E}[X(t)]+B u(t)+\bar{B} \mathbb{E}[u(t)]\} \mathrm{d} t \\
\quad+\{C X(t)+\bar{C} \mathbb{E}[X(t)]+D u(t)+\bar{D} \mathbb{E}[u(t)]\} \mathrm{d} W(t), \quad t \geqslant 0
\end{array}\right. \\
X(0)=x,
\end{array}\right.
$$


with the cost functional

$$
J\left(x ; u_{1}(\cdot), u_{2}(\cdot)\right)=\mathbb{E} \int_{0}^{\infty}\left[\left\langle\left(\begin{array}{cc}
Q & S^{\top} \\
S & R
\end{array}\right)\left(\begin{array}{c}
X(t) \\
u(t)
\end{array}\right),\left(\begin{array}{c}
X(t) \\
u(t)
\end{array}\right)\right\rangle+\left\langle\left(\begin{array}{cc}
\bar{Q} & \bar{S}^{\top} \\
\bar{S} & \bar{R}
\end{array}\right)\left(\begin{array}{c}
\mathbb{E}[X(t)] \\
\mathbb{E}[u(t)]
\end{array}\right),\left(\begin{array}{c}
\mathbb{E}[X(t)] \\
\mathbb{E}[u(t)]
\end{array}\right)\right\rangle\right] \mathrm{d} t .
$$

Let

$$
\left\{\begin{array}{l}
A=\left(\begin{array}{cc}
-1 & -1 \\
0 & -3
\end{array}\right), \quad \bar{A}=\left(\begin{array}{cc}
-1 & 0 \\
-1 & -20
\end{array}\right), \quad B=\left(\begin{array}{ll}
1 & 0 \\
0 & 1
\end{array}\right), \quad \bar{B}=\left(\begin{array}{cc}
2.5 & 0 \\
0 & 0.5
\end{array}\right), \\
C=\left(\begin{array}{cc}
1 & 0.5 \\
0.5 & 1
\end{array}\right), \quad \bar{C}=\left(\begin{array}{cc}
0 & -0.5 \\
-0.5 & 0
\end{array}\right), \quad D=\left(\begin{array}{ll}
1 & 0 \\
0 & 1
\end{array}\right), \quad \bar{D}=\left(\begin{array}{cc}
0.5 & 0 \\
0 & 0.5
\end{array}\right), \\
Q=\left(\begin{array}{cc}
2.3070 & 0.7781 \\
0.7781 & 0.3123
\end{array}\right), \quad \bar{Q}=\left(\begin{array}{cc}
5.6930 & 0.7219 \\
0.7219 & 22.2317
\end{array}\right), \quad S=\left(\begin{array}{ll}
0 & 0 \\
0 & 0
\end{array}\right), \quad \bar{S}=\left(\begin{array}{ll}
0 & 0 \\
0 & 0
\end{array}\right), \\
R=\left(\begin{array}{cc}
2 & 0 \\
0 & -2
\end{array}\right), \quad \bar{R}=\left(\begin{array}{cc}
0.75 & 0 \\
0 & -0.5
\end{array}\right),
\end{array}\right.
$$

and from (2.15), we have

$$
\begin{cases}\widehat{A}=\left(\begin{array}{cc}
-2 & -1 \\
-1 & -23
\end{array}\right), \quad \widehat{B}=\left(\begin{array}{cc}
3.5 & 0 \\
0 & 1.5
\end{array}\right), & \widehat{C}=\left(\begin{array}{cc}
1 & 0 \\
0 & 1
\end{array}\right), \quad \widehat{D}=\left(\begin{array}{cc}
1.5 & 0 \\
0 & 1.5
\end{array}\right) \\
\widehat{Q}=\left(\begin{array}{cc}
8 & 1.5 \\
1.5 & 22.5440
\end{array}\right), \quad \widehat{S}=\left(\begin{array}{cc}
0 & 0 \\
0 & 0
\end{array}\right), \quad \widehat{R}=\left(\begin{array}{cc}
2.75 & 0 \\
0 & -2.5
\end{array}\right)\end{cases}
$$

First, we look at closed-loop representation of open-loop saddle point. According to (4.10), we have the following coupled AREs:

$$
\left\{\begin{array}{l}
P A+A^{\top} P+C^{\top} P C+Q-\left(P B+C^{\top} P D+S^{\top}\right) \Sigma_{o}^{\dagger}\left(B^{\top} P+D^{\top} P C+S\right)=0 \\
\widehat{P} \widehat{A}+\widehat{A}^{\top} \widehat{P}+\widehat{C}^{\top} P \widehat{C}+\widehat{Q}-\left(\widehat{P} \widehat{B}+\widehat{C}^{\top} P \widehat{D}+\widehat{S}^{\top}\right) \bar{\Sigma}_{o}^{\dagger}\left(\widehat{B}^{\top} \widehat{P}+\widehat{D}^{\top} P \widehat{C}+\widehat{S}\right)=0
\end{array}\right.
$$

where

$$
\Sigma_{o} \equiv R+D^{\top} P D=\left(\begin{array}{cc}
2 & 0 \\
0 & -2
\end{array}\right)+P, \quad \bar{\Sigma}_{o} \equiv \widehat{R}+\widehat{D}^{\top} P \widehat{D}=\left(\begin{array}{cc}
2.75 & 0 \\
0 & -2.5
\end{array}\right)+2.25 P .
$$

Then, solving (5.3) yields

$$
P=\left(\begin{array}{cc}
1 & 0 \\
0 & 0.1
\end{array}\right), \quad \widehat{P}=\left(\begin{array}{cc}
1 & 0 \\
0 & 0.5
\end{array}\right)
$$

The above solutions satisfy (4.7) and (4.8). Further, since $\Sigma_{o}$ and $\bar{\Sigma}_{o}$ are invertible, we have

$$
\left\{\begin{array}{l}
\mathscr{R}\left(B^{\top} P+D^{\top} P C+S\right) \subseteq \mathscr{R}\left(\Sigma_{o}\right), \quad \mathscr{R}\left(\widehat{B}^{\top} \widehat{P}+\widehat{D}^{\top} P \widehat{C}+\widehat{S}\right) \subseteq \mathscr{R}\left(\bar{\Sigma}_{o}\right), \\
\Theta^{*}=-\Sigma_{o}^{-1}\left(B^{\top} P+D^{\top} P C+S\right)=\left(\begin{array}{cc}
-0.6667 & -0.1667 \\
0.0263 & 0.1053
\end{array}\right) \\
\bar{\Theta}^{*}=-\bar{\Sigma}_{o}^{-1}\left(\widehat{B}^{\top} \widehat{P}+\widehat{D}^{\top} P \widehat{C}+\widehat{S}\right)=\left(\begin{array}{cc}
-1 & 0 \\
0 & 0.3956
\end{array}\right)
\end{array}\right.
$$


According to Proposition A.5 in [23], the system $[A, \bar{A}, C, \bar{C} ; B, \bar{B}, D, \bar{D}]$ above is MF- $L^{2}$-stabilizable, and $(\Theta, \bar{\Theta}) \in \mathscr{S}[A, \bar{A}, C, \bar{C} ; B, \bar{B}, D, \bar{D}]$ if and only if there exist $P_{0}>0$ and $\bar{P}_{0}>0$, such that

$$
\left\{\begin{aligned}
& (A+B \Theta) P_{0}+P_{0}(A+B \Theta)^{\top}+(C+D \Theta) P_{0}(C+D \Theta)^{\top}+(\widehat{C}+\widehat{D} \bar{\Theta}) \bar{P}_{0}(\widehat{C}+\widehat{D} \bar{\Theta})^{\top} \\
= & {\left[\left(\begin{array}{cc}
-1 & -1 \\
0 & -3
\end{array}\right)+\Theta\right] P_{0}+P_{0}\left[\left(\begin{array}{cc}
-1 & -1 \\
0 & -3
\end{array}\right)+\Theta\right]^{\top}+\left[\left(\begin{array}{cc}
1 & 0.5 \\
0.5 & 1
\end{array}\right)+\Theta\right] P_{0}\left[\left(\begin{array}{cc}
1 & 0.5 \\
0.5 & 1
\end{array}\right)+\Theta\right]^{\top} } \\
& +\left[\left(\begin{array}{cc}
1 & 0 \\
0 & 1
\end{array}\right)+\left(\begin{array}{cc}
1.5 & 0 \\
0 & 1.5
\end{array}\right) \bar{\Theta}\right] \bar{P}_{0}\left[\left(\begin{array}{cc}
1 & 0 \\
0 & 1
\end{array}\right)+\left(\begin{array}{cc}
1.5 & 0 \\
0 & 1.5
\end{array}\right) \bar{\Theta}\right]^{\top}<0, \\
(\widehat{A}+\widehat{B} \bar{\Theta}) \bar{P}_{0}+\bar{P}_{0}(\widehat{A}+\widehat{B} \bar{\Theta})^{\top} & \\
= & {\left.\left[\begin{array}{cc}
-2 & -1 \\
-1 & -23
\end{array}\right)+\left(\begin{array}{cc}
3.5 & 0 \\
0 & 1.5
\end{array}\right) \bar{\Theta}\right] \bar{P}_{0}+\bar{P}_{0}\left[\left(\begin{array}{cc}
-2 & -1 \\
-1 & -23
\end{array}\right)+\left(\begin{array}{cc}
3.5 & 0 \\
0 & 1.5
\end{array}\right) \bar{\Theta}\right]^{\top}<0 . }
\end{aligned}\right.
$$

Substituting the above $\Theta^{*}$ and $\bar{\Theta}^{*}$ of (5.4) into (5.5), we have

$$
P_{0}=\left(\begin{array}{cc}
0.5742 & -0.0424 \\
-0.0424 & 0.4258
\end{array}\right)>0, \quad \bar{P}_{0}=\left(\begin{array}{cc}
0.8185 & -0.0377 \\
-0.0377 & 0.1815
\end{array}\right)>0,
$$

such that the negative definiteness conditions of (5.5) hold. Then $\left(\Theta^{*}, \bar{\Theta}^{*}\right)$ above is an MF- $L^{2}$-stabilizer of the system $[A, \bar{A}, C, \bar{C} ; B, \bar{B}, D, \bar{D}]$. Now, we solve corresponding systems (4.11)-(4.14) to get $\left(\eta_{o}(\cdot), \zeta_{o}(\cdot)\right)$ and $\bar{\eta}_{o}(\cdot)$. From (4.15), the closed-loop representation of this open-loop saddle point can be given by

$$
\begin{aligned}
u^{*}(\cdot)= & -\Sigma_{o}^{-1}\left(B^{\top} P+D^{\top} P C+S\right)\left(X^{*}(\cdot)-\mathbb{E}\left[X^{*}(\cdot)\right]\right)-\bar{\Sigma}_{o}^{-1}\left(\widehat{B}^{\top} \widehat{P}+\widehat{D}^{\top} P \widehat{C}+\widehat{S}\right) \mathbb{E}\left[X^{*}(\cdot)\right] \\
& -\Sigma_{o}^{-1}\left\{B^{\top}\left(\eta_{o}(\cdot)-\mathbb{E}\left[\eta_{o}(\cdot)\right]\right)+D^{\top}\left(\zeta_{o}(\cdot)-\mathbb{E}\left[\zeta_{o}(\cdot)\right]\right)\right\}-\bar{\Sigma}_{o}^{-1}\left\{\widehat{B}^{\top} \bar{\eta}_{o}(\cdot)+\widehat{D}^{\top} \mathbb{E}\left[\zeta_{o}(\cdot)\right]\right\},
\end{aligned}
$$

where $X^{*}(\cdot) \in \mathscr{X}[0, \infty)$ is the solution to (5.1) corresponding to $u^{*}(\cdot)$.

Next, we consider the closed-loop saddle point. Note that the corresponding system of generalized AREs (4.17) reads

$$
\left\{\begin{array}{l}
0=P_{c} A+A^{\top} P_{c}+C^{\top} P_{c} C+Q-\left(P_{c} B+C^{\top} P_{c} D+S^{\top}\right) \Sigma_{c}^{\dagger}\left(B^{\top} P_{c}+D^{\top} P_{c} C+S\right), \\
0=\widehat{P}_{c} \widehat{A}+\widehat{A}^{\top} \widehat{P}_{c}+\widehat{C}^{\top} P_{c} \widehat{C}+\widehat{Q}-\left(\widehat{P}_{c} \widehat{B}+\widehat{C}^{\top} P_{c} \widehat{D}+\widehat{S}^{\top}\right) \bar{\Sigma}_{c}^{\dagger}\left(\widehat{B}^{\top} \widehat{P}_{c}+\widehat{D}^{\top} P_{c} \widehat{C}+\widehat{S}\right),
\end{array}\right.
$$

with

$$
\left\{\begin{aligned}
\Sigma_{c} & \equiv R+D^{\top} P_{c} D=\left(\begin{array}{cc}
2 & 0 \\
0 & -2
\end{array}\right)+P_{c}, \\
\bar{\Sigma}_{c} & \equiv \widehat{R}+\widehat{D}^{\top} P_{c} \widehat{D}=\left(\begin{array}{cc}
2.75 & 0 \\
0 & -2.5
\end{array}\right)+2.25 P_{c} .
\end{aligned}\right.
$$

Then, solving (5.7) yields

$$
P_{c}=\left(\begin{array}{cc}
1 & 0 \\
0 & 0.1
\end{array}\right), \quad \widehat{P}_{c}=\left(\begin{array}{cc}
1 & 0 \\
0 & 0.5
\end{array}\right)
$$

Thus,

$$
\begin{cases}R_{11}+D_{1}^{\top} P_{c} D_{1}=3 \geqslant 0, & \widehat{R}_{11}+\widehat{D}_{1}^{\top} P_{c} \widehat{D}_{1}=5 \geqslant 0 \\ R_{22}+D_{2}^{\top} P_{c} D_{2}=-1.9 \leqslant 0, & \widehat{R}_{22}+\widehat{D}_{2}^{\top} P_{c} \widehat{D}_{2}=-2.275 \leqslant 0 .\end{cases}
$$


Also, the range condition

$$
\mathscr{R}\left(B^{\top} P_{c}+D^{\top} P_{c} C+S\right) \subseteq \mathscr{R}\left(\Sigma_{c}\right), \quad \mathscr{R}\left(\widehat{B}^{\top} \widehat{P}_{c}+\widehat{D}^{\top} P_{c} \widehat{C}+\widehat{S}\right) \subseteq \mathscr{R}\left(\bar{\Sigma}_{c}\right)
$$

holds automatically since $\Sigma_{c}$ and $\bar{\Sigma}_{c}$ are invertible. We see that

$$
\left\{\begin{array}{l}
\Theta^{*}=-\Sigma_{c}^{-1}\left(B^{\top} P_{c}+D^{\top} P_{c} C+S\right)=\left(\begin{array}{cc}
-0.6667 & -0.1667 \\
0.0263 & 0.1053
\end{array}\right), \\
\bar{\Theta}^{*}=-\bar{\Sigma}_{c}^{-1}\left(\widehat{B}^{\top} \widehat{P}_{c}+\widehat{D}^{\top} P_{c} \widehat{C}+\widehat{S}\right)=\left(\begin{array}{cc}
-1 & 0 \\
0 & 0.3956
\end{array}\right)
\end{array}\right.
$$

which coincides with (5.4). Hence, by Theorem 4.4, the problem admits a closed-loop saddle point. Solving corresponding backward systems (4.19)-(4.20), we can get $\left(\eta_{c}(\cdot), \zeta_{c}(\cdot)\right)$ and $\bar{\eta}_{c}(\cdot)$, the closed-loop saddle point is given by (5.9) and

$$
v^{*}(\cdot)=-\Sigma_{c}^{-1}\left\{B^{\top}\left(\eta_{c}(\cdot)-\mathbb{E}\left[\eta_{c}(\cdot)\right]\right)+D^{\top}\left(\zeta_{c}(\cdot)-\mathbb{E}\left[\zeta_{c}(\cdot)\right]\right)\right\}-\bar{\Sigma}_{c}^{-1}\left\{\widehat{B}^{\top} \bar{\eta}_{c}(\cdot)+\widehat{D}^{\top} \mathbb{E}\left[\zeta_{c}(\cdot)\right]\right\} .
$$

Since $P=P_{c}$ and $\widehat{P}=\widehat{P}_{c}$, we get $\Sigma=\Sigma_{c}, \bar{\Sigma}=\bar{\Sigma}_{c}$ and $\eta_{o}(\cdot)=\eta_{c}(\cdot), \zeta_{o}(\cdot)=\zeta_{c}(\cdot), \bar{\eta}_{o}(\cdot)=\bar{\eta}_{c}(\cdot)$. This implies that the closed-loop representation of the open-loop saddle point coincides with the closed-loop saddle point.

The following example shows that for the mean-field LQ non-zero sum stochastic differential game, it may happen that the closed-loop representations of open-loop Nash equilibria are different from the closed-loop Nash equilibria. However, the solutions to the system of algebraic Riccati equations for the closed-loop representation of open-loop Nash equilibrium are symmetric.

Example 5.2. Consider the following two-dimensional state equation

$$
\left\{\begin{array}{l}
\mathrm{d} X(t)=\left\{\begin{array}{l}
A X(t)+\bar{A} \mathbb{E}[X(t)]+B u(t)+\bar{B} \mathbb{E}[u(t)]\} \mathrm{d} t \\
\quad+\{C X(t)+\bar{C} \mathbb{E}[X(t)]+D u(t)+\bar{D} \mathbb{E}[u(t)]\} \mathrm{d} W(t), \quad t \geqslant 0
\end{array}\right. \\
X(0)=x,
\end{array}\right.
$$

with the cost functional

$$
\left\{\begin{aligned}
J_{1}\left(x ; u_{1}(\cdot), u_{2}(\cdot)\right)=\mathbb{E} \int_{0}^{\infty}[ & \left\langle\left(\begin{array}{cc}
Q_{1} & S_{1}^{\top} \\
S_{1} & R_{1}
\end{array}\right)\left(\begin{array}{c}
X(t) \\
u(t)
\end{array}\right),\left(\begin{array}{c}
X(t) \\
u(t)
\end{array}\right)\right\rangle \\
& \left.+\left\langle\left(\begin{array}{cc}
\bar{Q}_{1} & \bar{S}_{1}^{\top} \\
\bar{S}_{1} & \bar{R}_{1}
\end{array}\right)\left(\begin{array}{c}
\mathbb{E}[X(t)] \\
\mathbb{E}[u(t)]
\end{array}\right),\left(\begin{array}{c}
\mathbb{E}[X(t)] \\
\mathbb{E}[u(t)]
\end{array}\right)\right\rangle\right] \mathrm{d} t \\
J_{2}\left(x ; u_{1}(\cdot), u_{2}(\cdot)\right)=\mathbb{E} \int_{0}^{\infty}[ & \left\langle\left(\begin{array}{cc}
Q_{2} & S_{2}^{\top} \\
S_{2} & R_{2}
\end{array}\right)\left(\begin{array}{c}
X(t) \\
u(t)
\end{array}\right),\left(\begin{array}{c}
X(t) \\
u(t)
\end{array}\right)\right\rangle \\
& \left.+\left\langle\left(\begin{array}{cc}
\bar{Q}_{2} & \bar{S}_{2}^{\top} \\
\bar{S}_{2} & \bar{R}_{2}
\end{array}\right)\left(\begin{array}{c}
\mathbb{E}[X(t)] \\
\mathbb{E}[u(t)]
\end{array}\right),\left(\begin{array}{c}
\mathbb{E}[X(t)] \\
\mathbb{E}[u(t)]
\end{array}\right)\right\rangle\right] \mathrm{d} t .
\end{aligned}\right.
$$


We let

$$
\left\{\begin{array}{l}
A=\left(\begin{array}{cc}
-1 & -1 \\
0 & -1
\end{array}\right), \quad \bar{A}=\left(\begin{array}{cc}
-1 & 0 \\
-1 & -1
\end{array}\right), \quad B=\left(\begin{array}{ll}
1 & 0 \\
0 & 1
\end{array}\right), \quad \bar{B}=\left(\begin{array}{cc}
2.5 & 0 \\
0 & 0.5
\end{array}\right), \\
C=\left(\begin{array}{cc}
1 & 0.5 \\
0.5 & 1
\end{array}\right), \quad \bar{C}=\left(\begin{array}{cc}
0 & -0.5 \\
-0.5 & 0
\end{array}\right), \quad D=\left(\begin{array}{ll}
1 & 0 \\
0 & 1
\end{array}\right), \quad \bar{D}=\left(\begin{array}{cc}
0.5 & 0 \\
0 & 0.5
\end{array}\right), \\
Q_{1}=\left(\begin{array}{cc}
2.85 & 0.9 \\
0.9 & 2.475
\end{array}\right), \quad Q_{2}=\left(\begin{array}{cc}
1.35 & 0.4 \\
0.4 & 2.5375
\end{array}\right), \quad \bar{Q}_{1}=\left(\begin{array}{cc}
6.0324 & 0.6 \\
0.6 & 0.325
\end{array}\right), \\
\bar{Q}_{2}=\left(\begin{array}{cc}
7.15 & 1.6 \\
1.6 & 2.8625
\end{array}\right), \quad S_{1}=S_{2}=\left(\begin{array}{ll}
0 & 0 \\
0 & 0
\end{array}\right), \quad \bar{S}_{1}=\bar{S}_{2}=\left(\begin{array}{cc}
0 & 0 \\
0 & 0
\end{array}\right) \\
R_{1}=\left(\begin{array}{ll}
1 & 0 \\
0 & 1
\end{array}\right), \quad R_{2}=\left(\begin{array}{cc}
1 & 0 \\
0 & 1.5
\end{array}\right), \quad \bar{R}_{1}=\left(\begin{array}{ll}
1 & 0 \\
0 & 1
\end{array}\right), \quad \bar{R}_{2}=\left(\begin{array}{cc}
0.5 & 0 \\
0 & 0
\end{array}\right),
\end{array}\right.
$$

and from (2.15), we have

$$
\left\{\begin{array}{l}
\widehat{A}=\left(\begin{array}{cc}
-2 & -1 \\
-1 & -2
\end{array}\right), \quad \widehat{B}=\left(\begin{array}{cc}
3.5 & 0 \\
0 & 1.5
\end{array}\right), \quad \widehat{C}=\left(\begin{array}{ll}
1 & 0 \\
0 & 1
\end{array}\right), \quad \widehat{D}=\left(\begin{array}{cc}
1.5 & 0 \\
0 & 1.5
\end{array}\right), \quad \widehat{Q}_{1}=\left(\begin{array}{cc}
8.8824 & 1.5 \\
1.5 & 2.8
\end{array}\right), \\
\widehat{Q}_{2}=\left(\begin{array}{cc}
8.5 & 2 \\
2 & 5.4
\end{array}\right), \quad \widehat{S}_{1}=\widehat{S}_{2}=\left(\begin{array}{ll}
0 & 0 \\
0 & 0
\end{array}\right), \quad \widehat{R}_{1}=\left(\begin{array}{ll}
2 & 0 \\
0 & 2
\end{array}\right), \quad \widehat{R}_{2}=\left(\begin{array}{cc}
1.5 & 0 \\
0 & 1.5
\end{array}\right) .
\end{array}\right.
$$

To look at the closed-loop representation of open-loop Nash equilibria, we solve the corresponding (3.33)-(3.34) to get

$$
P_{1}=\left(\begin{array}{cc}
1 & 0 \\
0 & 1
\end{array}\right), \quad P_{2}=\left(\begin{array}{cc}
0.5 & 0 \\
0 & 1
\end{array}\right), \quad \widehat{P}_{1}=\left(\begin{array}{cc}
1 & 0 \\
0 & 0.5
\end{array}\right), \quad \widehat{P}_{2}=\left(\begin{array}{ll}
1 & 0 \\
0 & 1
\end{array}\right)
$$

which are symmetric. Since

$$
\left(\begin{array}{cc}
R_{111}+D_{1}^{\top} P_{1} D_{1} & R_{112}+D_{1}^{\top} P_{1} D_{2} \\
R_{221}+D_{2}^{\top} P_{2} D_{1} & R_{222}+D_{2}^{\top} P_{2} D_{2}
\end{array}\right)=\left(\begin{array}{cc}
2 & 0 \\
0 & 2.5
\end{array}\right)
$$

and

$$
\left(\begin{array}{ll}
\widehat{R}_{111}+\widehat{D}_{1}^{\top} P_{1} \widehat{D}_{1} & \widehat{R}_{112}+\widehat{D}_{1}^{\top} P_{1} \widehat{D}_{2} \\
\widehat{R}_{221}+\widehat{D}_{2}^{\top} P_{2} \widehat{D}_{1} & \widehat{R}_{222}+\widehat{D}_{2}^{\top} P_{2} \widehat{D}_{2}
\end{array}\right)=\left(\begin{array}{cc}
4.25 & 0 \\
0 & 3.75
\end{array}\right)
$$

are invertible, it follows that

$$
\Theta^{* *}=\left(\begin{array}{cc}
-1 & -0.25 \\
-0.2 & -0.8
\end{array}\right), \quad \bar{\Theta}^{* *}=\left(\begin{array}{cc}
-1.1765 & 0 \\
0 & -0.8
\end{array}\right) .
$$

Also, it follows from $(3.29)-(3.30)$, we can get $(\eta(\cdot), \zeta(\cdot))$ and $\bar{\eta}(\cdot)$. Then, from $(3.27)$, we have

$$
v^{* *}(\cdot)=-\boldsymbol{\Sigma}^{-1} \mathbf{J}^{\top}\left\{\mathbf{B}^{\top}(\eta(\cdot)-\mathbb{E}[\eta(\cdot)])+\mathbf{D}^{\top}(\zeta(\cdot)-\mathbb{E}[\zeta(\cdot)])\right\}-\overline{\boldsymbol{\Sigma}}^{-1} \mathbf{J}^{\top}\left\{\widehat{\mathbf{B}}^{\top} \bar{\eta}(\cdot)+\widehat{\mathbf{D}}^{\top} \mathbb{E}[\zeta(\cdot)]\right\} .
$$

By Theorem 3.3, making use of (3.27)-(3.30), the problem admits an open-loop Nash equilibrium $u^{*}(\cdot)$ for any initial state $x \in \mathbb{R}^{n}$. And it has the closed-loop representation (2.7) with $\left(\Theta^{* *}, \bar{\Theta}^{* *}, v^{* *}(\cdot)\right)$ given by $(5.13)$ and (5.14). 
Next, we consider the closed-loop Nash equilibria. According to Proposition A.5 in [23], system $[A, \bar{A}, C, \bar{C}$; $B, \bar{B}, D, \bar{D}]$ above is MF- $L^{2}$-stabilizable, and $(\Theta, \bar{\Theta}) \in \mathscr{S}[A, \bar{A}, C, \bar{C} ; B, \bar{B}, D, \bar{D}]$ if and only if there exist $P_{0}>0$ and $\bar{P}_{0}>0$, such that

$$
\left\{\begin{aligned}
& (A+B \Theta) P_{0}+P_{0}(A+B \Theta)^{\top}+(C+D \Theta) P_{0}(C+D \Theta)^{\top}+(\widehat{C}+\widehat{D} \bar{\Theta}) \bar{P}_{0}(\widehat{C}+\widehat{D} \bar{\Theta})^{\top} \\
= & {\left[\left(\begin{array}{cc}
-1 & -1 \\
0 & -1
\end{array}\right)+\Theta\right] P_{0}+P_{0}\left[\left(\begin{array}{cc}
-1 & -1 \\
0 & -1
\end{array}\right)+\Theta\right]^{\top}+\left[\left(\begin{array}{cc}
1 & 0.5 \\
0.5 & 1
\end{array}\right)+\Theta\right] P_{0}\left[\left(\begin{array}{cc}
1 & 0.5 \\
0.5 & 1
\end{array}\right)+\Theta\right]^{\top} } \\
& +\left[\left(\begin{array}{cc}
1 & 0 \\
0 & 1
\end{array}\right)+\left(\begin{array}{cc}
1.5 & 0 \\
0 & 1.5
\end{array}\right) \bar{\Theta}\right] \bar{P}_{0}\left[\left(\begin{array}{cc}
1 & 0 \\
0 & 1
\end{array}\right)+\left(\begin{array}{cc}
1.5 & 0 \\
0 & 1.5
\end{array}\right) \bar{\Theta}\right]^{\top}<0 \\
(\widehat{A}+\widehat{B} \bar{\Theta}) \bar{P}_{0}+\bar{P}_{0}(\widehat{A}+\widehat{B} \bar{\Theta})^{\top} & \\
= & {\left.\left[\begin{array}{ll}
-2 & -1 \\
-1 & -2
\end{array}\right)+\left(\begin{array}{cc}
3.5 & 0 \\
0 & 1.5
\end{array}\right) \bar{\Theta}\right] \bar{P}_{0}+\bar{P}_{0}\left[\left(\begin{array}{cc}
-2 & -1 \\
-1 & -2
\end{array}\right)+\left(\begin{array}{cc}
3.5 & 0 \\
0 & 1.5
\end{array}\right) \bar{\Theta}\right]^{\top}<0 . }
\end{aligned}\right.
$$

For $i=1,2$, we solve the corresponding (3.35)-(3.37) to get

$$
\begin{gathered}
\left\{\begin{array}{cl}
P_{1}=\left(\begin{array}{cc}
0.9949 & -0.0168 \\
-0.0168 & 0.9201
\end{array}\right), & P_{2}=\left(\begin{array}{cc}
0.6255 & -0.0104 \\
-0.0104 & 1.01741
\end{array}\right), \\
\widehat{P}_{1}=\left(\begin{array}{cc}
1.0023 & -0.0155 \\
-0.0155 & 0.6472
\end{array}\right), & \widehat{P}_{2}=\left(\begin{array}{cc}
0.8919 & 0.0126 \\
0.0126 & 0.9964
\end{array}\right),
\end{array}\right. \\
\begin{cases}\Sigma_{1} \triangleq R_{111}+D_{1}^{\top} P_{1} D_{1}=1.9949, & \Sigma_{2} \triangleq R_{222}+D_{2}^{\top} P_{2} D_{2}=2.5174, \\
\bar{\Sigma}_{1} \triangleq \widehat{R}_{111}+\widehat{D}_{1}^{\top} P_{1} \widehat{D}_{1}=4.2386, & \bar{\Sigma}_{2} \triangleq \widehat{R}_{222}+\widehat{D}_{2}^{\top} P_{2} \widehat{D}_{2}=3.7891,\end{cases}
\end{gathered}
$$

and

$$
\Theta^{*}=\left(\begin{array}{cc}
-0.9949 & -0.2393 \\
-0.1979 & -0.8072
\end{array}\right), \quad \bar{\Theta}^{*}=\left(\begin{array}{cc}
-1.1798 & 0.0117 \\
-0.0082 & -0.7971
\end{array}\right)
$$

Substituting the above $\Theta^{*}$ and $\bar{\Theta}^{*}$ of (5.16) into (5.15), we can get

$$
P_{0}=\left(\begin{array}{cc}
0.3323 & -0.0865 \\
-0.0865 & 0.3365
\end{array}\right)>0, \quad \bar{P}_{0}=\left(\begin{array}{cc}
0.1196 & -0.0327 \\
-0.0327 & 0.2115
\end{array}\right)>0 .
$$

such that the negative definiteness conditions of (5.15) hold. Then $\left(\Theta^{*}, \bar{\Theta}^{*}\right)$ above is an MF- $L^{2}$-stabilizer of the system $[A, \bar{A}, C, \bar{C} ; B, \bar{B}, D, \bar{D}]$. Hence, by Theorem 3.7 , the problem admits a closed-loop Nash equilibrium.

Now, since

$$
B\left(\Theta^{*}-\Theta^{* *}\right)=\left(\begin{array}{cc}
0.0051 & 0.0107 \\
0.0021 & -0.0072
\end{array}\right) \neq 0
$$

by Remark 2.7, we see that the closed-loop representation of the open-loop Nash equilibrium and the closed-loop Nash equilibrium are intrinsically different.

From this example, we see that the solutions to system (3.33)-(3.34), which come from the open-loop Nash equilibrium, may be symmetric, but they are different from the solution to the system of AREs (3.35)-(3.37), which come from the closed-loop Nash equilibrium. It is obvious that the closed-loop representation of the open-loop Nash equilibrium is different from the closed-loop Nash equilibrium. 
The following example shows that for the closed-loop representation of open-loop Nash equilibria of the mean-field LQ non-zero sum stochastic differential game, it may happen that the solutions to the system of AREs may be asymmetric when the system $[A, \bar{A}, C, \bar{C} ; B, \bar{B}, D, \bar{D}]$ is MF- $L^{2}$-stabilizable. And, the closed-loop representation of the open-loop Nash equilibrium is different from the closed-loop Nash equilibrium.

Example 5.3. Consider the two-dimensional state equation of form (5.11) with cost functionals (5.12), but we let

$$
\left\{\begin{array}{l}
A=\left(\begin{array}{cc}
-1 & -1 \\
0 & -1
\end{array}\right), \quad \bar{A}=\left(\begin{array}{cc}
-1 & 0 \\
-1 & -1
\end{array}\right), \quad B=\left(\begin{array}{ll}
0 & 0 \\
0 & 0
\end{array}\right), \quad \bar{B}=\left(\begin{array}{ll}
0 & 0 \\
0 & 0
\end{array}\right) \\
C=\left(\begin{array}{ll}
0 & 0 \\
0 & 0
\end{array}\right), \quad \bar{C}=\left(\begin{array}{ll}
0 & 0 \\
0 & 0
\end{array}\right), \quad D=\left(\begin{array}{ll}
1 & 0 \\
0 & 1
\end{array}\right), \quad \bar{D}=\left(\begin{array}{ll}
1 & 0 \\
0 & 1
\end{array}\right) \\
Q_{1}=\left(\begin{array}{ll}
2 & 1 \\
1 & \frac{3}{2}
\end{array}\right), \quad Q_{2}=\left(\begin{array}{ll}
1 & \frac{1}{2} \\
\frac{1}{2} & 3
\end{array}\right), \quad \bar{Q}_{1}=\left(\begin{array}{cc}
\frac{83}{44} & 1 \\
1 & \frac{105}{44}
\end{array}\right), \quad \bar{Q}_{2}=\left(\begin{array}{cc}
3 & \frac{3}{2} \\
\frac{3}{2} & \frac{16}{11}
\end{array}\right) \\
S_{1}=\left(\begin{array}{cc}
0 & 0 \\
\sqrt{\frac{5}{2}} & 0
\end{array}\right), \quad S_{2}=\left(\begin{array}{cc}
0 & 0 \\
0 & \sqrt{\frac{5}{2}}
\end{array}\right), \quad \bar{S}_{1}=\bar{S}_{2}=\left(\begin{array}{ll}
0 & 0 \\
0 & 0
\end{array}\right), \\
R_{1}=\left(\begin{array}{ll}
1 & 0 \\
0 & 1
\end{array}\right), \quad R_{2}=\left(\begin{array}{ll}
1 & 0 \\
0 & \frac{3}{2}
\end{array}\right), \quad \bar{R}_{1}=\left(\begin{array}{ll}
1 & 0 \\
0 & 1
\end{array}\right), \quad \bar{R}_{2}=\left(\begin{array}{ll}
\frac{1}{2} & 0 \\
0 & 0
\end{array}\right)
\end{array}\right.
$$

From (2.15), we have

$$
\left\{\begin{array}{l}
\widehat{A}=\left(\begin{array}{ll}
-2 & -1 \\
-1 & -2
\end{array}\right), \quad \widehat{B}=\left(\begin{array}{ll}
0 & 0 \\
0 & 0
\end{array}\right), \quad \widehat{C}=\left(\begin{array}{ll}
0 & 0 \\
0 & 0
\end{array}\right), \quad \widehat{D}=\left(\begin{array}{ll}
2 & 0 \\
0 & 2
\end{array}\right) \\
\widehat{Q}_{1}=\left(\begin{array}{cc}
\frac{171}{44} & 2 \\
2 & \frac{171}{44}
\end{array}\right), \quad \widehat{Q}_{2}=\left(\begin{array}{cc}
4 & 2 \\
2 & \frac{49}{11}
\end{array}\right), \quad \widehat{S}_{1}=\left(\begin{array}{cc}
0 & 0 \\
\sqrt{\frac{5}{2}} & 0
\end{array}\right), \quad \widehat{S}_{2}=\left(\begin{array}{cc}
0 & 0 \\
0 & \sqrt{\frac{5}{2}}
\end{array}\right) \\
\widehat{R}_{1}=\left(\begin{array}{ll}
2 & 0 \\
0 & 2
\end{array}\right), \quad \widehat{R}_{2}=\left(\begin{array}{cc}
\frac{3}{2} & 0 \\
0 & \frac{3}{2}
\end{array}\right) .
\end{array}\right.
$$

To look at the closed-representation of open-loop Nash equilibria, we solve the corresponding (3.33)-(3.34) to get

$$
P_{1}=\left(\begin{array}{cc}
1 & -\frac{1}{2} \\
0 & 1
\end{array}\right), \quad P_{2}=\left(\begin{array}{cc}
\frac{1}{2} & 0 \\
0 & 1
\end{array}\right), \quad \widehat{P}_{1}=\left(\begin{array}{cc}
1 & -\frac{5}{44} \\
0 & 1
\end{array}\right), \quad \widehat{P}_{2}=\left(\begin{array}{ll}
1 & 0 \\
0 & 1
\end{array}\right)
$$

We see that $P_{1}$ and $\widehat{P}_{1}$ are not symmetric. Since

$$
\left(\begin{array}{ll}
R_{111}+D_{1}^{\top} P_{1} D_{1} & R_{112}+D_{1}^{\top} P_{1} D_{2} \\
R_{221}+D_{2}^{\top} P_{2} D_{1} & R_{222}+D_{2}^{\top} P_{2} D_{2}
\end{array}\right)=\left(\begin{array}{cc}
2 & -\frac{1}{2} \\
0 & \frac{5}{2}
\end{array}\right)
$$

and

$$
\left(\begin{array}{ll}
\widehat{R}_{111}+\widehat{D}_{1}^{\top} P_{1} \widehat{D}_{1} & \widehat{R}_{112}+\widehat{D}_{1}^{\top} P_{1} \widehat{D}_{2} \\
\widehat{R}_{221}+\widehat{D}_{2}^{\top} P_{2} \widehat{D}_{1} & \widehat{R}_{222}+\widehat{D}_{2}^{\top} P_{2} \widehat{D}_{2}
\end{array}\right)=\left(\begin{array}{cc}
6 & -2 \\
0 & \frac{11}{2}
\end{array}\right)
$$


are invertible, it follows that

$$
\Theta^{* *}=\left(\begin{array}{cc}
0 & -0.1581 \\
0 & -0.6325
\end{array}\right), \quad \bar{\Theta}^{* *}=\left(\begin{array}{cc}
0 & -0.0958 \\
0 & -0.2875
\end{array}\right) .
$$

Then, similar to Example 5.2, making use of Theorem 3.3, we see that the problem admits an open-loop Nash equilibrium for any initial state $x \in \mathbb{R}^{n}$. And it has the closed-loop representation of form (2.7).

Next, we consider the closed-loop Nash equilibria. According to Proposition A.5 in [23], the system $[A, \bar{A}, C, \bar{C}$; $B, \bar{B}, D, \bar{D}]$ above is MF- $L^{2}$-stabilizable, and $(\Theta, \bar{\Theta}) \in \mathscr{S}[A, \bar{A}, C, \bar{C} ; B, \bar{B}, D, \bar{D}]$ if and only if there exist $P_{0}>0$ and $\bar{P}_{0}>0$, such that

$$
\left\{\begin{array}{l}
(A+B \Theta) P_{0}+P_{0}(A+B \Theta)^{\top}+(C+D \Theta) P_{0}(C+D \Theta)^{\top}+(\widehat{C}+\widehat{D} \bar{\Theta}) \bar{P}_{0}(\widehat{C}+\widehat{D} \bar{\Theta})^{\top} \\
=\left[\left(\begin{array}{cc}
-1 & -1 \\
0 & -1
\end{array}\right)+\Theta\right] P_{0}+P_{0}\left[\left(\begin{array}{cc}
-1 & -1 \\
0 & -1
\end{array}\right)+\Theta\right]^{\top}+\Theta P_{0} \Theta^{\top}+\left(\begin{array}{ll}
2 & 0 \\
0 & 2
\end{array}\right) \bar{\Theta} \bar{P}_{0} \bar{\Theta}^{\top}\left(\begin{array}{ll}
2 & 0 \\
0 & 2
\end{array}\right)<0 \\
(\widehat{A}+\widehat{B} \bar{\Theta}) \bar{P}_{0}+\bar{P}_{0}(\widehat{A}+\widehat{B} \bar{\Theta})^{\top}=\left(\begin{array}{ll}
-2 & -1 \\
-1 & -2
\end{array}\right) \bar{P}_{0}+\bar{P}_{0}\left(\begin{array}{ll}
-2 & -1 \\
-1 & -2
\end{array}\right)^{\top}<0 .
\end{array}\right.
$$

For $i=1,2$, we solve corresponding (3.35)-(3.37) to get

$$
\begin{gathered}
\begin{cases}P_{1}=\left(\begin{array}{cc}
1 & -0.4955 \\
-0.4955 & 1.7645
\end{array}\right), & P_{2}=\left(\begin{array}{cc}
0.5 & 0 \\
0 & 1.0226
\end{array}\right), \\
\widehat{P}_{1}=\left(\begin{array}{cc}
1.0647 & -0.1861 \\
-0.1861 & 1.2327
\end{array}\right), & \widehat{P}_{2}=\left(\begin{array}{cc}
1.0016 & -0.0032 \\
-0.0032 & 1.0111
\end{array}\right),\end{cases} \\
\begin{cases}\Sigma_{1} \triangleq R_{111}+D_{1}^{\top} P_{1} D_{1}=2, & \Sigma_{2} \triangleq R_{222}+D_{2}^{\top} P_{2} D_{2}=2.5226, \\
\bar{\Sigma}_{1} \triangleq \widehat{R}_{111}+\widehat{D}_{1}^{\top} P_{1} \widehat{D}_{1}=6, & \bar{\Sigma}_{2} \triangleq \widehat{R}_{222}+\widehat{D}_{2}^{\top} P_{2} \widehat{D}_{2}=5.5902 .\end{cases}
\end{gathered}
$$

and

$$
\Theta^{*}=\left(\begin{array}{cc}
0 & -0.1553 \\
0 & -0.6268
\end{array}\right), \quad \bar{\Theta}^{*}=\left(\begin{array}{cc}
0 & -0.0934 \\
0 & -0.2828
\end{array}\right)
$$

Substituting the above $\Theta^{*}$ and $\bar{\Theta}^{*}$ of (5.19) into (5.18), we can get

$$
P_{0}=\left(\begin{array}{cc}
0.3082 & -0.0910 \\
-0.0910 & 0.3148
\end{array}\right)>0, \quad \bar{P}_{0}=\left(\begin{array}{cc}
0.1901 & -0.0721 \\
-0.0721 & 0.1870
\end{array}\right)>0 .
$$

such that the negative definiteness conditions of (5.18) hold. Then $\left(\Theta^{*}, \bar{\Theta}^{*}\right)$ above is an MF- $L^{2}$-stabilizer of the system $[A, \bar{A}, C, \bar{C} ; B, \bar{B}, D, \bar{D}]$. Hence, by Theorem 3.7 , the problem admits a closed-loop Nash equilibrium.

Finally, similar to Example 5.2, we easily check that the closed-loop representation of the optimal open-loop strategy and the optimal closed-loop strategy are intrinsically different.

From this example, we see that the solutions to system (3.33)-(3.34), which come from the open-loop saddle point, may be asymmetric, but the solutions to system (3.35)-(3.37), which come from the closed-loop saddle point, are still symmetric. It is obvious that the closed-loop representation of the open-loop Nash equilibrium is different from the closed-loop Nash equilibrium. 


\section{Concluding REMARKS}

In this paper, we have presented a systematic theory for two-person non-zero sum differential games of meanfield SDEs with quadratic performance indexes in $[0, \infty)$. The case of two-person zero-sum, which is also new, has been treated as a special case. Our results cover several existing ones in the literature for infinite horizon problems, including LQ optimal control problems of mean-field type [23], two-person zero-sum LQ stochastic differential games (without mean-field terms) [50], LQ optimal control problem (without mean-field terms), and the equivalence between the open-loop solvability and the closed-loop solvability for stochastic LQ problem in $[0, \infty)[46]$. Finally, we have to leave the following question open: Is the existence of the open-loop and closed-loop saddle points equivalent for the mean-field LQ two-person zero-sum stochastic differential game in an infinite horizon? Some related papers can be seen in Sun [42], Sun-Li-Yong [43], Sun-Yong [45], Yu [55], etc. We will research this topic in the future.

Acknowledgements. The authors would like to thank the editor and two anonymous referees for their critical and suggestive comments which lead to the current much better looking version. Thanks also go to Dr. Jingrui Sun of Department of Mathematics, Southern University of Science and Technology for quite a few discussions.

This work was carried out during the stay of Jingtao Shi at University of Central Florida, from December 2019 to July 2020. He would like to thank the invitation of Professor Jiongmin Yong, the hospitality of the Department of Mathematics, University of Central Florida, and the financial support from the China Scholarship Council.

\section{REFERENCES}

[1] M. Ait Rami and X.Y. Zhou, Linear matrix inequalities, Riccati equations, and indefinite stochastic linear quadratic controls. IEEE Trans. Automat. Control 45 (2000) 1131-1143.

[2] M. Ait Rami, X.Y. Zhou and J.B. Moore, Well-posedness and attainability of indifinite stochastic linear quadratic control in infinite time horizon. Syst. Control Lett. 41 (2000) 123-133.

[3] N.U. Ahmed and X. Ding, Controlled McKean-Vlasov equations. Commun. Appl. Anal. 5 (2001) 183-206.

[4] D. Andersson and B. Djehiche, A maximum principle for stochastic control of SDE's of mean-field type. Appl. Math. Optim. 63 (2011) 341-356.

[5] B.D.O. Anderson and J.B. Moore, Optimal Control: Linear Quadratic Methods. Prentice Hall, Englewood Cliffs, NJ (1989).

[6] A. Bensoussan, J. Frehse and S.C.P. Yam, Mean field games and mean field type control theory. Springer, New York (2013).

[7] A. Bensoussan, K.C.J. Sung and S.C.P. Yam, Linear-quadratic time-inconsistent mean-field games. Dyn. Games Appl. 3 (2013) $537-552$.

[8] A. Bensoussan, K.C.J. Sung, S.C.P. Yam and S.P. Yung, Linear-quadratic mean-field games. J. Optim. Theory Appl. 169 (2016) 496-529.

[9] A. Bensoussan, S.C.P. Yam and Z. Zhang, Well-posedness of mean-field type forward-backward stochastic differential equations. Stoch. Proc. Appl. 125 (2015) 3327-3354.

[10] R. Buckdahn, B. Djehiche and J. Li, A general stochastic maximum principle for SDEs of mean-field type. Appl. Math. Optim. 64 (2011) 197-216.

[11] R. Buckdahn, J. Li and J. Ma, A stochastic maximum principle for general mean-field systems. Appl. Math. Optim. 74 (2016) 507-534.

[12] R. Buckdahn, J. Li and J. Ma, A mean-field stochastic control problem with partial observations. Ann. Probab. 27 (2017) 3201-3245.

[13] R. Buckdahn, J. Li and S.G. Peng, Mean-field backward stochastic differential equations and related partial differential equations. Stoch. Proc. Appl. 119 (2009) 3113-3154.

[14] R. Buckdahn, J. Li, S.G. Peng and C. Rainer, Mean-field stochastic differential equations and associated PDEs. Ann. Probab. 45 (2017) 824-878.

[15] R. Carmona and F. Delarue, Mean field forward-backward stochastic differential equations. Electr. Commun. Probab. 18 (2013) 1-15.

[16] R. Carmona and F. Delarue, Forward-backward stochastic differential equations and controlled McKean-Vlasov dynamics. Ann. Probab. 43 (2015) 2647-2700.

[17] T. Chan, Dynamics of the McKean-Vlasov equation. Ann. Probab. 22 (1994) 431-441.

[18] S.N. Cohen and V. Fedyashov, Nash equilibria for nonzero-sum ergodic stochastic differential games. J. Appl. Probab. 54 (2017) 977-994.

[19] B. Djehiche and M. Tembine, A characterization of sub-game perfect equilibria for SDEs of mean-field type. Dyn. Games. Appl. 6 (2016) 55-81.

[20] B. Djehiche, H. Tembine and R. Tempone, A stochastic maximum principle for risk-sensitive mean-field type control. IEEE Trans. Autom. Control 60 (2015) 2640-2649. 
[21] J.J.A. Hosking, A stochsastic maximum principle for a stochastic differential game of a mean-field type. Appl. Math. Optim. 66 (2012) 415-454.

[22] J. Huang, X. Li and T. Wang, Mean-field linear-quadratic-Gaussian (LQG) games for stochastic integral systems. IEEE Trans. Autom. Control 61 (2016) 2670-2675.

[23] J. Huang, X. Li, and J. Yong, A linear-quadratic optimal control problem for mean-field stochastic differential equations in infinite horizon. Math. Control Rel. Fields 5 (2015) 97-139.

[24] M. Huang, R.P. Malhamé and P.E. Caines, Large population stochastic dynamic games: Closed-loop McKean-Vlasov systems and the Nash certainty equivalence principle. Commun. Inf. Syst. 6 (2006) 221-251.

[25] M. Kac, Foundations of kinetic theory. Proc. Third Berkeley Symp. Math. Stat. Probab. 3 (1956) 171-197.

[26] J.M. Lasry and P.L. Lions, Mean field games. Jpn. J. Math. 2 (2007) 229-260.

[27] J. Li, Stochastic maximum principle in the mean-field controls. Automatica 48 (2012) 366-373.

[28] N. Li, X. Li and Z. Yu, Indefinite mean-field type linear-quadratic stochastic optimal control problems. Automatica 122 (2020) 109267.

[29] X. Li, J. Shi and J. Yong, Mean-field linear-quadratic stochastic differential games in an infinite horizon. http://arxiv.org/ abs/2007.06130v1.

[30] X. Li, J. Sun and J. Xiong, Linear quadratic optimal control problems for mean-field backward stochastic differential equations. Appl. Math. Optim. 80 (2018) 223-250.

[31] X. Li, J. Sun and J. Yong, Mean-field stochastic linear quadratic optimal control problems: closed-loop solvability. Probab. Uncer. ES Quan. Risk 1 (2016) 1-24.

[32] H.P. McKean, A class of Markov processes associated with nonlinear parabolic equations. Proc. Natl. Acad. Sci. USA 56 (1966) 1907-1911.

[33] T. Meyer-Brandis, B. Øksendal, and X.Y. Zhou, A mean-field stochastic maximum principle via Malliavin calculus. Stochastics 84 (2012) 643-666.

[34] E. Miller and H. Pham, Linear-quadratic McKean-Vlasov stochastic differential games, in vol. 164 of Modeling, Stochastic Control, Optimization, and Applications, IMA Vol. Math. Appl., edited by G. Yin and Q. Zhang. Springer Nature, Switzerland (2019) 451-481.

[35] J. Moon, Linear-quadratic mean-field stochastic zero-sum differential games. Automatica 120 (2020) 109067.

[36] L. Mou and J. Yong, Two-person zero-sum linear quadratic stochastic differential games by a Hilbert space method. J. Indu. Mana. Optim. 2 (2006) 93-115.

[37] H. Pham and X. Wei, Dynamic programming for optimal control of stochastic McKean-Vlasov dynamics. SIAM J. Control Optim. 55 (2017) 1069-1101.

[38] H. Pham and X. Wei, Bellman equation and viscosity solutions for mean-field stochastic control problem. ESAIM: COCV 24 (2018) 437-461.

[39] R. Penrose, A generalized inverse of matrices. Proc. Cambr. Philos. Soc. 52 (1955) 17-19.

[40] M. Scheutzow, Uniqueness and non-uniqueness of solutions of Vlasov-McKean equations. J. Aust. Math. Soc., Ser. A 43 (1987) 246-256.

[41] J. Sun, Mean-field stochastic linear quadratic optimal control problems: Open-loop solvabilities. ESAIM: COCV 23 (2017) 1099-1127.

[42] J. Sun, Two-person zero-sum stochastic linear-quadratic differential games. http://arxiv.org/abs/2005.11701v1.

[43] J. Sun, X. Li and J. Yong, Open-loop and closed-loop solvabilities for stochastic linear quadratic optimal control problems. SIAM J. Control Optim. 54 (2016) 2274-2308.

[44] J. Sun, H. Wang and Z. Wu, Mean-field linear-quadratic stochastic differential games. http://arxiv.org/abs/2101.00412v1.

[45] J. Sun and J. Yong, Linear quadratic stochastic differential games: Open-loop and closed-loop saddle points. SIAM J. Control Optim. 52 (2014) 4082-4121.

[46] J. Sun and J. Yong, Stochastic linear quadratic optimal control problems in infinite horizon. Appl. Math. Optim. 78 (2018) $145-183$.

[47] J. Sun and J. Yong, Linear quadratic stochastic two-person nonzero-sum differential games: Open-loop and closed-loop Nash equilibria. Stoch. Proc. Appl. 129 (2019) 381-418.

[48] J. Sun and J. Yong, Stochastic Linear-Quadratic Optiml Control Theory: Open-Loop and Closed-Loop Solutions. Springer (2020).

[49] J. Sun and J. Yong, Stochastic Linear-Quadratic Optiml Control Theory: Differential Games and Mean-Field Problems. Springer (2020).

[50] J. Sun, J. Yong and S.G. Zhang, Linear quadratic stochastic two-person zero-sum differential games in an infinite horizon. ESAIM: COCV 22 (2016) 743-769.

[51] R. Tian, Z. Yu and R. Zhang, A closed-loop saddle point for zero-sum linear-quadratic stochastic differential games with mean-field type. Syst. Control Lett. 136 (2020) 104624.

[52] J. Yong, A leader-follower stochastic linear quadratic differential games. SIAM J. Control Optim. 41 (2002) $1015-1041$.

[53] J. Yong, A linear-quadratic optimal control problem for mean-field stochastic differential equations. SIAM J. Control Optim. 51 (2013) 2809-2838.

[54] J. Yong, Linear-quadratic optimal control problems for mean-field stochastic differential equations-time-consistent solutions. Trans. Amer. Math. Soc. 369 (2017) 5467-5523.

[55] Z. Yu, An optimal feedback control-strategy pair for zero-sum linear-quadratic stochastic differential game: the Riccati equation approach. SIAM J. Control Optim. 55 (2015) 2141-2167. 